\title{
Wall crossing from Boltzmann black hole halos
}

\author{
Jan Manschot, ${ }^{a}$ Boris Pioline $^{b}$ and Ashoke Sen ${ }^{c}$ \\ anstitut de Physique Théorique, CEA Saclay, CNRS-URA 2306, \\ 91191 Gif sur Yvette, France \\ ${ }^{b}$ Laboratoire de Physique Théorique et Hautes Energies, CNRS UMR 7589, \\ Université Pierre et Marie Curie, \\ 4 place Jussieu, 75252 Paris cedex 05, France \\ ${ }^{c}$ Harish-Chandra Research Institute, \\ Chhatnag Road, Jhusi, Allahabad 211019, India
}

E-mail: jan.manschot@cea.fr, pioline@lpthe.jussieu.fr, sen@hri.res.in

ABSTRACT: A key question in the study of $\mathcal{N}=2$ supersymmetric string or field theories is to understand the decay of BPS bound states across walls of marginal stability in the space of parameters or vacua. By representing the potentially unstable bound states as multi-centered black hole solutions in $\mathcal{N}=2$ supergravity, we provide two fully general and explicit formulæ for the change in the (refined) index across the wall. The first, "Higgs branch" formula relies on Reineke's results for invariants of quivers without oriented loops, specialized to the Abelian case. The second, "Coulomb branch" formula results from evaluating the symplectic volume of the classical phase space of multi-centered solutions by localization. We provide extensive evidence that these new formulæ agree with each other and with the mathematical results of Kontsevich and Soibelman (KS) and Joyce and Song (JS). The main physical insight behind our results is that the Bose-Fermi statistics of individual black holes participating in the bound state can be traded for Maxwell-Boltzmann statistics, provided the (integer) index $\Omega(\gamma)$ of the internal degrees of freedom carried by each black hole is replaced by an effective (rational) index $\bar{\Omega}(\gamma)=\sum_{m \mid \gamma} \Omega(\gamma / m) / m^{2}$. A similar map also exists for the refined index. This observation provides a physical rationale for the appearance of the rational Donaldson-Thomas invariant $\bar{\Omega}(\gamma)$ in the works of KS and JS.

Keywords: Black Holes in String Theory, Topological Strings, Brane Dynamics in Gauge Theories, Solitons Monopoles and Instantons

ArXiv EPRINT: 1011.1258 


\section{Contents}

1 Introduction and summary 2

2 Boltzmannian view of the wall-crossing $\quad 8$

2.1 BPS states in $\mathcal{N}=2$ supergravity 8

2.2 Wall crossing: preliminaries 9

$\begin{array}{lll}2.3 & \text { Bose-Fermi statistics to Maxwell-Boltzmann statistics } & 10\end{array}$

2.4 General wall-crossing formula and charge conservation 12

2.5 Semi-primitive wall-crossing from Boltzmann gas of black hole molecules 13

$\begin{array}{ll}2.6 & \text { Refined 'index' in supergravity } \\ \end{array}$

3 Multi-black hole bound states and quiver quantum mechanics $\quad 18$

$\begin{array}{lll}3.1 & \text { Higgs branch analysis } & 19\end{array}$

3.2 Coulomb branch analysis 23

3.3 Comparison of the results of Higgs branch and Coulomb branch analysis 29

4 Wall crossing from the Kontsevich-Soibelman formula 32

4.1 The KS formula 33

4.2 Charge conservation from KS formula 34

$\begin{array}{lll}4.3 & \text { Primitive wall-crossing } & 35\end{array}$

4.4 Generic 3-body and 4-body contributions 35

4.5 Semi-primitive wall-crossing formulae and generalizations 37

$\begin{array}{lll}4.5 .1 & \text { Order one } & 37\end{array}$

$\begin{array}{lll}4.5 .2 & \text { Order two } & 38\end{array}$

$\begin{array}{lll}4.5 .3 & \text { Order three } & 40\end{array}$

$\begin{array}{lll}4.6 & \gamma_{12}>0 \text { case } & 42\end{array}$

4.7 Refined wall-crossing and motivic invariants 42

4.8 Semi-primitive refined wall-crossings and its generalizations 44

$\begin{array}{lll}4.9 & \text { KS vs. supergravity } & 45\end{array}$

5 Wall-crossing from the Joyce-Song formula 46

$\begin{array}{lll}5.1 & \text { Statement of the JS formula } & 47\end{array}$

5.2 Index of supersymmetric bound states from the JS formula 50

5.3 Generic 2-body, 3-body and 4-body contributions 51

5.4 Semi-primitive wall-crossing formula from JS 53

$\begin{array}{ll}\text { A Wall crossing formulae in special cases } & 55\end{array}$

$\begin{array}{ll}\text { B D6-D0 bound states } & 60\end{array}$

$\begin{array}{ll}\text { C Seiberg-Witten spectra and generalizations } & 65\end{array}$

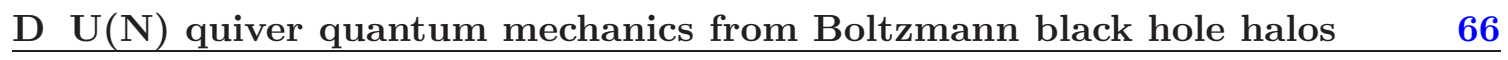




\section{Introduction and summary}

In quantum field theories and string theory vacua with extended supersymmetry, the spectrum of BPS states can sometimes be determined exactly in a weakly coupled region of the space of parameters (or vacua). In extrapolating the BPS spectrum to another point in parameter space, one must be wary of two issues: BPS states may pair up and disappear, and single particle states may decay into the continuum of multi-particle states. The first issue can be evaded by considering a suitable index $\Omega\left(\gamma ; t^{a}\right)$, where $\gamma$ is the vector of electric and magnetic charges carried by the state and $t^{a}$ parametrizes the value of the couplings (or moduli), designed such that contributions from long multiplets cancel. The index $\Omega\left(\gamma ; t^{a}\right)$ is then a piecewise constant function of the parameters $t^{a}$. To deal with the second problem, it is important to understand how $\Omega\left(\gamma ; t^{a}\right)$ changes across certain codimension-one subspaces of the parameter space, known as walls of marginal stability, where a single-particle BPS state becomes marginally unstable against decay into two (or more) BPS states [1-6].

Initial progress in this direction for four-dimensional string vacua came from supergravity, where BPS states are represented by (in general multi-centered) classical black hole solutions. Since the class of multi-centered solutions potentially unstable at a certain wall of marginal stability exists only on one side of the wall [7-9], the discontinuity $\Delta \Omega(\gamma)$ in $\Omega\left(\gamma, t^{a}\right)$ is equal to the index of the multi-centered solutions with total charge $\gamma$, up to a sign depending whether one enters or leaves the side on which these solutions exist [10]. Based on this physical picture, one easily finds that the jump of the index in the simplest case, where the only configuration that may appear or disappear across the wall is a two-centered solution with primitive charge vectors $\gamma_{1}, \gamma_{2}$, is given by the "primitive wall-crossing formula" [10]:

$$
\Delta \Omega(\gamma)=(-1)^{\gamma_{12}+1}\left|\gamma_{12}\right| \Omega\left(\gamma_{1}\right) \Omega\left(\gamma_{2}\right)
$$

With some more effort one can also compute $\Delta \Omega(\gamma)$ in "semi-primitive" cases, where the relevant multi-centered solutions which appear or disappear across the wall of marginal stability are halos of black holes with charges in multiple of $\gamma_{2}$, orbiting around a core of charge $\gamma_{1}[10]$.

While applying this method to the general "non-primitive" case seemed out of reach up until now, a breakthrough came from the mathematical front, with the works of Kontsevich and Soibelman (KS) [11, 12] and Joyce and Song (JS) [13-15]. In these works, general formulæ were derived for the discontinuity of generalized Donaldson-Thomas (DT) invariants under changes of stability conditions. It is generally believed that generalized DT invariants are the appropriate mathematical embodiment of the physical BPS invariants. Although the KS and JS wall-crossing formulæ look very different, there is by now much evidence that they are equivalent. ${ }^{1}$ Since these two formulæ appeared, much efforts have been devoted towards interpreting, deriving and checking these wall-crossing formulæ in

\footnotetext{
${ }^{1}$ We have been informed by D. Joyce of a general argument showing the equivalence of the KS and JS wall-crossing formulae. The equality of the DT invariants as defined by KS and JS seems, on the other hand, less firmly established.
} 
various physical settings [16-24]. Our goal in this paper is to rederive the wall-crossing formula using multi-centered black hole solutions in supergravity and extend the original black hole halo picture to the general "non-primitive" case. We also carry out extensive comparisons between our formulæ and those of KS and JS.

One intriguing aspect of the KS and JS wall-crossing formulæ is the appearance of two types of BPS-invariants, an integer-valued invariant $\Omega$ (roughly $\operatorname{Tr}(-1)^{F}$ ) and a rationalvalued invariant $\bar{\Omega}$. The two are related by the "multi-cover formula"

$$
\bar{\Omega}(\gamma)=\sum_{m \mid \gamma} m^{-2} \Omega(\gamma / m),
$$

where the sum runs over all positive integers $m$ such that $\gamma / m$ lies in the charge lattice. We shall take (1.2) as the definition of $\bar{\Omega}$. Similar divisor sums appear in various instances in quantum field theory, e.g. in Schwinger's computation of pair creation in an external electric field (see e.g. eqs. (4.118) and (4.119) in [25]), and in enumerative geometry, e.g. the multi-cover formulæ for Gromov-Witten invariants [26], which indeed are naturally understood from pair creation arguments $[27,28]$. The rational invariants $\bar{\Omega}$ also arose in constructions of modular invariant black hole partition functions consistent with wallcrossing [29-31], and in studies of D-brane instanton corrections to the hypermultiplet moduli space metric $[18,32,33]$.

For the purposes of computing the jump in the BPS spectrum, the rational invariants turn out to be especially convenient. Indeed, one consequence of the JS/KS wall-crossing formulæ is that the variation $\Delta \bar{\Omega}(\gamma)$ of the rational invariants across a wall of marginal stability, when expressed in terms of the rational invariants $\bar{\Omega}\left(\gamma^{\prime}\right)$ on one side of the wall, involves only "charge conserving" terms, i.e. sums of products of $\bar{\Omega}\left(\gamma_{i}\right)$ for different $\gamma_{i}$ such that $\sum_{i} \gamma_{i}=\gamma$. In contrast, the variation of the integer invariants $\Delta \Omega(\gamma)$, expressed in terms of the integer invariants $\Omega(\gamma)$, does not satisfy this property, and as a result, involves considerably more terms. Needless to say, physical charge is conserved no matter what invariant one chooses to consider.

Our main new insight, at the basis of the results presented below, is the following physical explanation of this phenomenon. In computing $\Delta \Omega(\gamma)$ from the index associated with a $n$-centered black hole solution carrying total charge $\gamma=\sum_{i=1}^{n} \gamma_{i}$, each center must be treated as a point-like particle carrying $\left|\Omega\left(\gamma_{i}\right)\right|$ internal states. When some of the $\gamma_{i}$ coincide, the corresponding centers must in addition obey Bose or Fermi statistics, depending on the sign of $\Omega\left(\gamma_{i}\right)$. As a result of (anti-)symmetrizing the many-body wavefunction, the total index associated with such a configuration involves, in addition to the product $\prod_{i=1}^{n} \Omega\left(\gamma_{i}\right)$, terms of lower degree in $\Omega\left(\gamma_{i}\right)$ - e.g. two identical bosons of degeneracy $\Omega$ will give a degeneracy of $\Omega(\Omega+1) / 2$. The terms of lower degree in $\Omega$ violate the charge conservation property defined above. However, due to special properties of the interactions between centers (namely, the no-force condition between centers with mutually local charges), we show that it is possible to map the problem of computing the index of multi-centered black holes with individual centers satisfying Bose-Fermi statistics to an equivalent problem where the centers satisfy instead Maxwell-Boltzmann statistics. In this Boltzmannian reformulation, each center carries an effective (in general non integer) 
index $\bar{\Omega}(\gamma)$ related to $\Omega(\gamma)$ via (1.2), and charge conservation is manifest. This provides a physical rationale of the charge conservation property of the wall-crossing formula written in terms of the rational invariants $\bar{\Omega}$.

The same argument generalizes for the refined 'index' $\Omega_{\text {ref }}(\gamma, y)$, defined roughly as $\operatorname{Tr}(-1)^{F} y^{2 J_{3}}$, which keeps track of the angular momentum of the BPS states. However this refined 'index' is only protected (i.e. immune to contributions of long multiplets) in the presence of a $\mathrm{SU}(2)_{R}$ symmetry [22], which may exists in $\mathcal{N}=2$ supersymmetric field theory, but not in string theory or supergravity. Nevertheless, it is a very useful quantity in intermediate stages of the analysis, as its variation can be computed using localization methods, and the variation of the usual index $\Omega(\gamma)$ can be obtained by carefully taking the $y \rightarrow 1$ limit at the end of the computation. Moreover, despite the fact that this refined 'index' may in general depend on both vector multiplets and hypermultiplets (in particular, its value may be different at weak and strong coupling), one may still investigate the variation of $\Omega_{\text {ref }}(\gamma, y)$ across lines of marginal stability in vector multiplet moduli space. In fact, KS have provided a wall-crossing formula for motivic Donaldson-Thomas invariants, which are conjectured to be equal to the refined invariants $\Omega_{\mathrm{ref}}(\gamma, y)$ at weak coupling, where the derived category description of D-branes is appropriate [34, 35]. Similarly, one may ask about the wall-crossing formula in the strong coupling region where the supergravity picture is appropriate. As for the standard index, we find that the variation $\Delta \Omega_{\mathrm{ref}}^{ \pm}(\gamma, y)$ can be computed by treating centers as Boltzmannian particles carrying internal states with an effective refined index

$$
\bar{\Omega}_{\mathrm{ref}}(\gamma, y) \equiv \sum_{m \mid \gamma} \frac{y-y^{-1}}{m\left(y^{m}-y^{-m}\right)} \Omega_{\mathrm{ref}}\left(\gamma / m, y^{m}\right) .
$$

In this formulation, charge conservation is again manifest. At $y=1, \Omega_{\text {ref }}(\gamma, y)$ reduces to $\Omega(\gamma)$ and the rational invariant (1.3) to (1.2).

While the arguments above rely on representing BPS states as multi-centered solutions in supergravity, it is clear that they extend to the case of $\mathcal{N}=2$ supersymmetric gauge theories which can be obtained as rigid limits of supergravity theories [36]. In general, we expect that BPS solitons in the Coulomb phase can be represented as classical multicentered solutions of the Abelian gauge theory at low energy, albeit singular ones. For the purposes of computing the wall-crossing, the singularity is irrelevant, and the problem can still be reduced to the quantum mechanics of point-like particles interacting by Coulomb law and scalar interactions. In particular, solitons with mutually local charges $\left(\left\langle\gamma_{1}, \gamma_{2}\right\rangle=0\right)$ do not interact, and the above Bose-Fermi/Boltzmann equivalence carries over.

Finally, it is worth pointing out that a similar phenomenon occurs for non-primitive wall-crossing in $\mathcal{N}=4$ supersymmetric string theories [37]. In this case only two-centered configurations contribute, and the only non-trivial effect comes from symmetrization [38, 39]. The variation of the index is thus given by the primitive wall-crossing formula (1.1), provided $\Omega(\gamma)$ is replaced by with $\tilde{\Omega}(\gamma)=\sum_{m \mid \gamma} \Omega(\gamma / m)$ in this formula. Note that in contrast to the effective index (1.2) relevant for $\mathcal{N}=2$ BPS states, the effective index $\tilde{\Omega}(\gamma)$ relevant for $\mathcal{N}=4$ dyons does not include any factor of $1 / m^{2}$ in its definition. This difference can be traced to the presence of extra fermion zero modes carried by a quarter 
BPS dyon in $\mathcal{N}=4$ supersymmetric theories. The trace over these fermionic zero modes produces an extra factor of $m^{2}$ in (1.2) compared to that for half-BPS dyons in $\mathcal{N}=2$ supersymmetric theories.

We shall now summarise our main results. Consider a wall of marginal stability on which the central charges $Z_{\gamma_{1}}$ and $Z_{\gamma_{2}}$ of two charge vectors $\gamma_{1}$ and $\gamma_{2}$ align. Assume further that, possibly after a change of basis in the lattice spanned by $\gamma_{1}$ and $\gamma_{2}$, BPS states carrying charge $M \gamma_{1}+N \gamma_{2}$ exist only for $(M \geq 0, N \geq 0)$ and $(M \leq 0, N \leq 0)$. Then on one side of the wall, which we call the chamber $c^{-}$, we have $\left\langle\gamma_{1}, \gamma_{2}\right\rangle \operatorname{Im}\left(Z_{\gamma_{1}} \bar{Z}_{\gamma_{2}}\right)>0$, and there are multi-centered bound states with individual centers carrying charges of the form $m_{i} \gamma_{1}+n_{i} \gamma_{2}$ with different integers $m_{i}, n_{i} \geq 0$. Here $\left\langle\gamma_{1}, \gamma_{2}\right\rangle$ is the symplectic inner product between $\gamma_{1}$ and $\gamma_{2}$. On the other side of the wall, called the chamber $c^{+}$, there are no bound states of this form. Let us denote by $\Omega^{ \pm}(\alpha)$ the index $\operatorname{Tr}^{\prime}(-1)^{2 J_{3}}$ on the two sides of the wall for a charge vector $\alpha=M \gamma_{1}+N \gamma_{2}$ with $M, N \geq 0$. ( $\operatorname{Tr}^{\prime}$ denotes the trace after removing the fermion zero modes associated with broken supersymmetries.) Then the physical reasoning outlined above shows that the wall-crossing formula, expressed in terms of the rational invariants (1.2), must take the form

$$
\bar{\Omega}^{-}(\gamma)-\bar{\Omega}^{+}(\gamma)=\sum_{n \geq 2} \sum_{\substack{\left\{\alpha_{1}, \ldots, \alpha_{n}\right\} \\ \gamma=\alpha_{1}+\cdots+\alpha_{n}}} \frac{g\left(\left\{\alpha_{i}\right\}\right)}{\left|\operatorname{Aut}\left(\left\{\alpha_{i}\right\}\right)\right|} \prod_{i=1}^{n} \bar{\Omega}^{+}\left(\alpha_{i}\right),
$$

where the sum runs over all possible unordered decompositions of $\alpha$ into vectors $\alpha_{1}, \ldots, \alpha_{n}$, each of which is a linear combination of $\gamma_{1}$ and $\gamma_{2}$ with non-negative integer coefficients. Here, $\left|\operatorname{Aut}\left(\left\{\alpha_{i}\right\}\right)\right|$ is the symmetry factor appropriate for Maxwell-Boltzmann statistics, namely the order of the subgroup of the permutation group of $n$ elements which preserves the ordered set $\left(\alpha_{1}, \ldots, \alpha_{n}\right)$, for a fixed (arbitrary) choice of ordering. ${ }^{2}$ Of course, one could instead decide to absorb this symmetry factor in the normalization of $g\left(\left\{\alpha_{i}\right\}\right)$. The point of the normalization chosen in (1.4) is that $g\left(\alpha_{1}, \ldots, \alpha_{n}\right)$ can now be identified as the index associated with an $n$-centered black hole configuration in supergravity, with the individual centers carrying charges $\alpha_{1}, \alpha_{2}, \ldots, \alpha_{n}$ and treated as distinguishable particles, and furthermore carrying no internal degeneracy. Clearly, the same considerations imply that the wall-crossing formula for refined invariants takes an analog form

$$
\bar{\Omega}_{\mathrm{ref}}^{-}(\gamma, y)-\bar{\Omega}_{\mathrm{ref}}^{+}(\gamma, y)=\sum_{n \geq 2} \sum_{\substack{\left\{\alpha_{1}, \ldots, \alpha_{n}\right\} \\ \gamma=\alpha_{1}+\cdots+\alpha_{n}}} \frac{g_{\mathrm{ref}}\left(\left\{\alpha_{i}\right\}, y\right)}{\left|\operatorname{Aut}\left(\left\{\alpha_{i}\right\}\right)\right|} \prod_{i=1}^{n} \bar{\Omega}_{\mathrm{ref}}^{+}\left(\alpha_{i}, y\right),
$$

where $g_{\text {ref }}\left(\left\{\alpha_{i}\right\}, y\right)$ computes the refined 'index' of the same $n$-centered black hole configuration, and reduces to $g\left(\left\{\alpha_{i}\right\}\right)$ at $y=1$. In order to complete the wall-crossing formula we need to specify the factor $g_{\text {ref }}\left(\left\{\alpha_{i}\right\}, y\right)$ (or its $y=1$ limit $g\left(\left\{\alpha_{i}\right\}\right)$ ). While these factors can be extracted from the KS and JS formulae, we shall present two novel ways for computing them, which we call the "Higgs branch" and the "Coulomb branch" formulae. We have checked in many cases the equivalence of these prescriptions with the KS and JS formulae, although we have not yet been able to prove the equivalence rigorously.

\footnotetext{
${ }^{2}$ Thus if the set $\left\{\alpha_{i}\right\}$ consists of $m_{1}$ copies of $\beta_{1}, m_{2}$ copies of $\beta_{2}$ etc. then $\left|\operatorname{Aut}\left(\left\{\alpha_{i}\right\}\right)\right|=\prod_{k} m_{k} !$.
} 
The "Higgs branch" formula is based on Denef's observation [8] that the spectrum of supersymmetric bound states of multi-centered black holes can be computed in the framework of quiver quantum mechanics. This description is appropriate at weak coupling, the arrows of the quiver describing the open strings stretched between two D-branes. Due to the fact that the charges carried by the various centers lie on a two-dimensional sublattice of the full charge lattice, the relevant quiver turns out to have no oriented loops. A formula for the motivic invariants of such quivers was given by Reineke in [40]. Furthermore, since the constituents of the bound states are to be treated as distinguishable particles without internal multiplicity, the relevant quiver carries dimension one vector spaces at each node (equivalently, corresponds to a $\mathrm{U}(1)^{n}$ gauge theory). Reineke's formula simplifies in this case, leading to

$$
g_{\mathrm{ref}}\left(\alpha_{1}, \ldots, \alpha_{n}, y\right)=(-y)^{-1+n-\sum_{i<j} \alpha_{i j}}\left(y^{2}-1\right)^{1-n} \sum_{\text {partitions }}(-1)^{s-1} y^{2 \sum_{a \leq b} \sum_{j<i} \alpha_{j i} m_{i}^{(a)}} m_{j}^{(b)} .
$$

Here we have denoted by $\alpha_{i j}=\left\langle\alpha_{i}, \alpha_{j}\right\rangle$ the symplectic inner product between the vectors $\alpha_{i}$ and $\alpha_{j}$, and have ordered the $\alpha_{i}$ 's such that $\left\langle\alpha_{i}, \alpha_{j}\right\rangle>0$ for $i<j$ (assuming that none of the vectors $\alpha_{i}$ coincide). The sum runs over all ordered partitions of $\left(\alpha_{1}+\cdots+\alpha_{n}\right)$ into $s$ vectors $\beta^{(a)}(1 \leq a \leq s, 1 \leq s \leq n)$ such that

1. $\sum_{a} \beta^{(a)}=\alpha_{1}+\cdots+\alpha_{n}$

2. $\beta^{(a)}=\sum_{i} m_{i}^{(a)} \alpha_{i}$ with $m_{i}^{(a)}=0$ or 1 for each $a, i$.

3. $\left\langle\sum_{a=1}^{b} \beta^{(a)}, \alpha_{1}+\cdots+\alpha_{n}\right\rangle>0 \quad \forall \quad b$ with $1 \leq b \leq s-1$

When some of the $\alpha_{i}$ 's coincide, the value of $g_{\text {ref }}\left(\alpha_{1}, \ldots, \alpha_{n}, y\right)$ can still be obtained from (1.6) by taking the limit $\alpha_{i} \rightarrow \alpha_{j}$ : even though the $\alpha_{i}$ 's are supposed to be valued in the two dimensional lattice spanned by $\gamma_{1}$ and $\gamma_{2}$, eq. (3.1) defines a continuous function of the $\alpha_{i}$ 's and this limit is well-defined. We have checked agreement with the KS and JS formulae for distinct $\alpha_{i}$ with $n \leq 5$, and in many cases where some of the $\alpha_{i}$ coincide. While it is not surprising that the Reineke formula is consistent with the JS formula (since the latter applies to moduli space of quiver representations), it is remarkable that Abelian quivers (i.e. quivers carrying a dimension-one vector space at each node) encode the complete information about wall-crossing. In appendix $\S \mathrm{D}$ we show that the index of certain non-Abelian quivers without oriented loops can be reduced to the Abelian case using the same black hole halo picture.

Our second way of computing $g_{\text {ref }}\left(\left\{\alpha_{i}\right\}, y\right)$ instead follows from quantizing the moduli space of multi-centered BPS solutions, as first proposed in [41]. This description is appropriate at strong coupling, when the classical supergravity description is valid, and is justified by the fact that the distance between the constituents diverges near the wall, leading to a decoupling between their internal degrees of freedom and the configurational degrees of freedom described by the classical moduli space $\mathcal{M}_{n}$ of $n$-centered BPS solutions. Using the fact that $\mathcal{M}_{n}$ carries a natural symplectic structure and a Hamiltonian action of $\mathrm{SU}(2)$, we evaluate the integral of $y^{2 J_{3}}$ over this classical phase space by localization. The 
fixed points of the action of $J_{3}$ on $\mathcal{M}_{n}$ are collinear configurations where all centers lie on the $z$-axis, with relative distances determined by

$$
\sum_{\substack{j=1 \\ j \neq i}}^{n} \frac{\alpha_{\sigma(i) \sigma(j)}}{z_{\sigma(j)}-z_{\sigma(i)}} \operatorname{sign}(j-i)=\Lambda \sum_{\substack{j=1 \\ j \neq i}}^{n} \alpha_{\sigma(i) \sigma(j)},
$$

where $\Lambda$ is a positive real constant which vanishes at the wall, and $\sigma$ is the permutation which determines the order of the centers along the axis, $z_{\sigma(i)}<z_{\sigma(j)}$ if $i<j$. In this way we arrive at the "Coulomb branch formula"

$$
g_{\mathrm{ref}}\left(\left\{\alpha_{i}\right\}, y\right)=(-1)^{\sum_{i<j} \alpha_{i j}+n-1}\left(y-y^{-1}\right)^{1-n} \sum_{\text {permutations } \sigma} s(\sigma) y^{\sum_{i<j} \alpha_{\sigma(i) \sigma(j)}},
$$

where the sum runs over the subset of the permutation group in $n$ elements for which the equations (1.7) admit a solution. The factor $s(\sigma)= \pm 1$ is a sign defined in (3.42), which originates from the determinant of the action of $J_{3}$ on the tangent space to the fixed point. While eq. (1.8) requires solving at least approximately the equations (1.7) (rather, finding the permutations $\sigma$ such that (1.7) admits a solution), it provides us with an economic way of determining $g_{\text {ref }}\left(\left\{\alpha_{i}\right\}, y\right)$, since each permutation has a unique $y$ dependence and hence there are no cancellations between different permutations. In contrast both the JS formula and "Higgs branch" formula (1.6) involves extensive cancellations between different terms. We shall in fact see in $\S 3.3$ that motivated by the Coulomb branch formula, one can find an algorithm to identify the uncancelled contributions in the Higgs branch formula without having to evaluate all the terms given in (1.6) and re-express the sign $s(\sigma)$ in a more convenient form (3.46).

The use of the rational invariants $\bar{\Omega}$ also allows us to use the KS formula to derive explicit formulas for the change in the index in some special cases. This includes sectors carrying charges of the form $2 \gamma_{1}+N \gamma_{2}$ and $3 \gamma_{1}+N \gamma_{2}$ for primitive vectors $\gamma_{1}, \gamma_{2}$ and arbitrary integer $N$. This generalizes the semi-primitive wall-crossing formula of [10] which describes the change in the index in the sector with charge $\gamma_{1}+N \gamma_{2}$, and some earlier results on higher-rank ADHM and DT invariants [42-44].

The rest of the paper is organised as follows. In $\S 2$ we describe how the problem of computing the index of multi-centered black holes can be mapped to an equivalent problem with the individual centers obeying Maxwell-Boltzmann statistics. We use this to derive some general properties of the wall-crossing formula e.g. charge conservation, and also reproduce the primitive and semi-primitive wall crossing formula. We also generalize the results to the case of refined index. In $\S 3$ we compute the index associated to $n$-centered black hole configurations in two different ways, first by mapping the problem to a quiver quantum mechanics and second by quantizing the classical phase space of multi-centered solutions. This leads to the Coulomb and Higgs branch formulae described above. In $\S 4$ we review the KS wall-crossing formula, and recast it in terms of the rational invariants $\bar{\Omega}$, making the charge conservation property manifest. We verify that the motivic KS formula agrees with the results of $\S 2$ and $\S 3$ in many cases, and obtain higher order generalizations of the semi-primitive wall-crossing formula. In $\S 5$ we review the wall-crossing formula due 
to Joyce and Song and compare it to the KS, Higgs branch and Coulomb formulae. We find agreement in all cases that we consider. In appendix A we illustrate the general wall crossing formulæ in some special cases. In appendix B we apply the results of $\S 4$ to analyze the spectrum of D0-D6 bound states on a Calabi-Yau 3-fold. In appendix C we check our results against the spectrum of BPS states in $\mathcal{N}=2 \mathrm{SU}(2)$ gauge theories. In appendix $\mathrm{D}$ we show how our Boltzmann gas picture allows one to express the Poincaré polynomial associated with quiver quantum mechanics with $\mathrm{U}(N)$ factors in terms of the Poincaré polynomial of Abelian quivers. This lends further support to the validity of the Boltzmann gas picture of multi-centered black holes.

\section{Boltzmannian view of the wall-crossing}

\subsection{BPS states in $\mathcal{N}=2$ supergravity}

We consider $\mathcal{N}=2$ supergravity in 4 dimensions, coupled to $n_{v}$ vector multiplets. Let $\mathcal{M}$ be the vector multiplet moduli space, parametrized by complex scalar fields $t^{a}, a=1, \ldots, n_{v}$, and $\Gamma$ be the lattice of electromagnetic charges. $\Gamma$ is a lattice of rank dimension $2 n_{v}+2$, equipped with an integer symplectic pairing. We choose a Lagrangian decomposition $\Gamma=$ $\Gamma_{m} \oplus \Gamma_{e}$, such that the symplectic pairing is given by

$$
\left\langle\gamma, \gamma^{\prime}\right\rangle=q_{\Lambda} p^{\Lambda}-q_{\Lambda}^{\prime} p_{\Lambda} \in \mathbb{Z}
$$

where $\gamma=\left(p^{\Lambda}, q_{\Lambda}\right), \gamma^{\prime}=\left(p^{\Lambda}, q_{\Lambda}^{\prime}\right)$. The mass of a BPS state with charge $\gamma$ is equal to the absolute value of the central charge $Z_{\gamma}$, defined by

$$
Z_{\gamma}=e^{\mathcal{K} / 2}\left(q_{\Lambda} X^{\Lambda}-p^{\Lambda} F_{\Lambda}\right)
$$

where $\mathcal{K}, X^{\Lambda}$ and $F_{\Lambda}$ are appropriate functions of the moduli fields $\left\{t^{a}\right\}$. Let $\mathcal{H}_{\gamma}\left(t^{a}\right)$ be the Hilbert space of states with charge $\gamma$ in the vacuum where the scalars asymptote to $t^{a}$ at spatial infinity. The index

$$
\Omega\left(\gamma ; t^{a}\right)=-\frac{1}{2} \operatorname{Tr}_{\mathcal{H}_{\gamma}\left(t^{a}\right)}(-1)^{2 J_{3}}\left(2 J_{3}\right)^{2}
$$

defines an integer $\Omega\left(\gamma ; t^{a}\right)$, which counts the number of BPS states with sign (the overall coefficient ensures that a half-hypermultiplet contributes one unit to $\left.\Omega\left(\gamma ; t^{a}\right)\right)$. Alternatively we could define the index as

$$
\Omega\left(\gamma ; t^{a}\right)=\operatorname{Tr}_{\mathcal{H}_{\gamma}\left(t^{a}\right)}^{\prime}(-1)^{2 J_{3}}
$$

where $\operatorname{Tr}^{\prime}$ denotes trace over BPS states, computed after removing the contribution from the fermion zero modes associated with the supersymmetries broken by the state. Mathematically, the BPS invariants $\left\{\Omega\left(\gamma ; t^{a}\right), \gamma \in \Gamma\right\}$ are the generalized Donaldson-Thomas (DT) invariants for the derived category of coherent sheaves (in type IIA on a Calabi-Yau threefold $\mathcal{X}$ ) or the Fukaya category (in type IIB on a Calabi-Yau threefold $\mathcal{X}$ ). 


\subsection{Wall crossing: preliminaries}

The BPS invariants $\Omega\left(\gamma ; t^{a}\right)$ are locally constant functions of $t^{a}$, but may jump on codimension one subspaces of $\mathcal{M}$ (line of marginal stability), where $\gamma$ can be written as the sum $\gamma=M \gamma_{1}+N \gamma_{2}$, where $M, N$ are two non-zero integers and $\gamma_{1}$ and $\gamma_{2}$ are two primitive (non-zero) vectors such that the phases of $Z_{\gamma_{1}}$ and $Z_{\gamma_{2}}$ are aligned. We denote the hyperplane where the phases of $\gamma_{1}, \gamma_{2}$ are aligned by $\mathcal{P}\left(\gamma_{1}, \gamma_{2}\right)$. Since the dependence of $\Omega\left(\gamma ; t^{a}\right)$ on $\left\{t^{a}\right\}$ is mild, we shall drop $t^{a}$ from the argument of $\Omega$ and use superscripts \pm to indicate which side of $\mathcal{P}\left(\gamma_{1}, \gamma_{2}\right)$ we are computing the index.

Clearly, $\mathcal{P}\left(\gamma_{1}, \gamma_{2}\right)$ depends only on the two-plane spanned by $\gamma_{1}$ and $\gamma_{2}$ inside $\Gamma$. For a given choice of $\gamma$ and of this two plane, $(M, N)$ and $\left(\gamma_{1}, \gamma_{2}\right)$ are uniquely defined up to a common action of $\mathrm{SL}(2, \mathbb{Z})$. We shall now make a special choice of $\left(\gamma_{1}, \gamma_{2}\right)$ such that there are no BPS states carrying charges of the form $M \gamma_{1}-N \gamma_{2}$ or $-M \gamma_{1}+N \gamma_{2}$ with $M, N>0$ [23]. For this reason it will be convenient to introduce the notation

$$
\tilde{\Gamma}: \quad\left\{M \gamma_{1}+N \gamma_{2}, \quad M, N \geq 0, \quad(M, N) \neq(0,0)\right\},
$$

and focus on BPS states carrying charge in $\tilde{\Gamma}$. For definiteness we shall choose $\left\langle\gamma_{1}, \gamma_{2}\right\rangle<0$.

We shall be considering the jump in the index $\Omega\left(M \gamma_{1}+N \gamma_{2}\right)$ across the wall $\mathcal{P}\left(\gamma_{1}, \gamma_{2}\right)$ for non-negative integers $M, N$. We shall denote by $Z_{\gamma}$ the central charge associated with the charge $\gamma$, and choose $\Omega^{+}$to describe the index in the chamber in which $\arg \left(Z_{\gamma_{1}}\right)>$ $\arg \left(Z_{\gamma_{2}}\right)$. In this case $\arg \left(Z_{M \gamma_{1}+N \gamma_{2}}\right)$ is greater (less) than $\arg \left(Z_{M^{\prime} \gamma_{1}+N^{\prime} \gamma_{2}}\right)$ if $M / N$ is greater (less) than $M^{\prime} / N^{\prime}$. We shall denote this chamber by $c_{+}$. For $\Omega^{-}$the ordering of $\arg \left(Z_{M \gamma_{1}+N \gamma_{2}}\right)$ is reversed, and the corresponding chamber will be called $c_{-}$. Using the fact that $\left\langle\gamma_{1}, \gamma_{2}\right\rangle<0$ we now get in the chamber $c_{+}$

$$
\left\langle M \gamma_{1}+N \gamma_{2}, M^{\prime} \gamma_{1}+N^{\prime} \gamma_{2}\right\rangle \operatorname{Im}\left(Z_{M \gamma_{1}+N \gamma_{2}} \bar{Z}_{M^{\prime} \gamma_{1}+N^{\prime} \gamma_{2}}\right)<0 .
$$

This is the condition under which two-centered bound states of black holes carrying charges $M \gamma_{1}+N \gamma_{2}$ and $M^{\prime} \gamma_{1}+N^{\prime} \gamma_{2}$ do not exist [7]. Thus $\Omega^{+}$'s label the index in the chamber in which there are no two centered black holes with each center carrying charge in $\tilde{\Gamma}$. Generalizing this argument (see $\S 3.2$ ) one can show that in the chamber $c^{+}$there are also no multi-centered black holes carrying charges of the form $\left(M_{i} \gamma_{1}+N_{i} \gamma_{2}\right)$ for different $\left(\left\{M_{i}\right\},\left\{N_{i}\right\}\right)$. In contrast $\Omega^{-}$'s label the index in the chamber where such bound states are present.

Note that $\Omega^{+}$can receive contribution both from single and multi-centered black holes, but these multi-centered configurations consist of centers whose charges lie outside the sublattice spanned by $\gamma_{1}$ and $\gamma_{2}$. Due to this the distances between the centers remain finite as the wall $\mathcal{P}\left(\gamma_{1}, \gamma_{2}\right)$ is approached. In contrast the distance between the centers carrying charges in $\tilde{\Gamma}$ - appearing in a configuration contributing to $\Omega^{-}$— goes to infinity in this limit. Thus the configurations which contribute to $\Omega^{+}$, even though not necessarily single centered black holes, can be treated as a single unit near this wall of marginal stability. For this reason we shall refer to $\Omega^{+}\left(M \gamma_{1}+N \gamma_{2}\right)$ as the index associated with a black hole molecule of charge $M \gamma_{1}+N \gamma_{2}$. Using this analogy, the full system, containing multiple molecules loosely bound to each other near the wall $\mathcal{P}\left(\gamma_{1}, \gamma_{2}\right)$, may be thought 
of as a molecular cluster. While the black hole molecule may itself be made of atoms (i.e. single centered black holes), the nature of these constituents is irrelevant for the problem at hand.

Our aim is to provide a wall-crossing formula which expresses $\Omega^{-}$in terms of $\Omega^{+}$. In supergravity the difference $\Omega^{-}-\Omega^{+}$is accounted for by the bound states of multiple black hole molecules carrying charges of the form $M_{i} \gamma_{1}+N_{i} \gamma_{2}$, since they contribute to $\Omega^{-}$but not to $\Omega^{+}$. Our goal in the rest of this section and the next section will be to use this viewpoint to derive the wall-crossing formula.

\subsection{Bose-Fermi statistics to Maxwell-Boltzmann statistics}

Let $\gamma_{0}$ be a primitive point on the charge lattice and let $d\left(s \gamma_{0}\right)$ be the number of bosonic states minus the number of fermionic states of a black hole molecule ${ }^{3}$ carrying charge $s \gamma_{0}$ moving in some appropriate background. We shall consider a system carrying total charge $k \gamma_{0}$ consisting of a gas of mutually non-interacting black hole molecules carrying charges $s \gamma_{0}$ for different integers $s$. A typical configuration will contain $m_{s}$ black hole molecules with charge $s \gamma_{0}$, subject to the constraint

$$
\sum_{s} s m_{s}=k
$$

The net contribution to the index from such configurations is given by

$$
N_{k}=\sum_{\substack{\left\{m_{s}\right\} \\ \sum_{s} s m_{s}=k}} \prod_{s}\left[\frac{1}{m_{s} !} \frac{\left(d\left(s \gamma_{0}\right)+m_{s}-1\right) !}{\left.\left(d\left(s \gamma_{0}\right)-1\right)\right) !}\right] .
$$

For bosons $d\left(s \gamma_{0}\right)>0$, and the above result follows from the fact that $m$ identical bosons occupying $d$ states produce a degeneracy of $d_{B}=d(d+1) \cdots(d+m-1) / m$ !. For fermions $d\left(s \gamma_{0}\right)<0$, and the result follows from the fact that $m$ fermions occupying $|d|$ states have total degeneracy $d_{F}=(|d|)(|d|-1) \cdots(|d|-m+1) / m$ ! and index $(-1)^{m} d_{F}=d(d+$ 1) $\cdots(d+m-1) / m$ !. It will be convenient to encode $(2.8)$ into a generating function

$$
\begin{aligned}
f(x)=\sum_{k} N_{k} x^{k} & =\sum_{\left\{m_{s}\right\}} \prod_{s} \frac{1}{m_{s} !} \frac{\left(d\left(s \gamma_{0}\right)+m_{s}-1\right) !}{\left.\left(d\left(s \gamma_{0}\right)-1\right)\right) !} x^{s m_{s}} \\
& =\prod_{s}\left(1-x^{s}\right)^{-d\left(s \gamma_{0}\right)} .
\end{aligned}
$$

We shall now prove that exactly the same contribution to the index is obtained if for each $\gamma$ we replace $d(\gamma)$ by

$$
\bar{d}(\gamma) \equiv \sum_{m \mid \gamma} m^{-1} d(\gamma / m)
$$

and treat the particles as obeying Maxwell-Boltzmann statistics rather than Bose or Fermi statistics. For this we calculate the generating function of the index of a gas of noninteracting Boltzmann black hole molecules carrying charges $s \gamma_{0}$ for different integers $s$.

\footnotetext{
${ }^{3}$ We exclude from this counting the fermionic zero modes associated with broken supersymmetry. A formal way of doing this is to use helicity supertraces $[45,46]$ instead of the Witten index.
} 
This is given by

$$
\begin{aligned}
g(x) & =\sum_{\left\{m_{s}\right\}} \prod_{s} \frac{1}{m_{s} !}\left(\bar{d}\left(s \gamma_{0}\right)\right)^{m_{s}} x^{s m_{s}}=\exp \left(\sum_{s} \bar{d}\left(s \gamma_{0}\right) x^{s}\right) \\
& =\exp \left(\sum_{s} \sum_{m \mid s} d\left(s \gamma_{0} / m\right) m^{-1} x^{s}\right)=\exp \left(\sum_{k} \sum_{m} d\left(k \gamma_{0}\right) m^{-1} x^{m k}\right) \\
& =\exp \left(-\sum_{k} d\left(k \gamma_{0}\right) \ln \left(1-x^{k}\right)\right)=\prod_{k}\left(1-x^{k}\right)^{-d\left(k \gamma_{0}\right)} .
\end{aligned}
$$

The $m_{s}$ ! in the denominator in the first line is the symmetry factor required for resolving the Gibbs paradox. Comparing (2.11) with (2.9) we see that the generating functions $f(x)$ and $g(x)$ are the same. Thus we are allowed to treat the black hole molecules as Boltzmann particles as long as we use the effective index $\bar{d}(\gamma)$.

In general $d\left(s \gamma_{0}\right)$ receives contribution from the intrinsic index $\Omega^{+}\left(s \gamma_{0}\right)$ of the black hole molecules and from the orbital degeneracy describing its motion in the background of other charges. The contribution to the orbital part of a black hole molecule of charge $s \gamma_{0}$ is expected not to be affected by the presence of the other black hole molecules carrying charges $l \gamma_{0}$ for any integer $l$ since the symplectic product $\left\langle k \gamma_{0}, l \gamma_{0}\right\rangle$ vanishes and as a consequence the particles are mutually noninteracting. In particular the repulsive electromagnetic interactions cancel against the attractive gravitational and scalar exchange interactions. ${ }^{4}$ On the other hand the orbital degeneracy is expected to depend on the background produced by other black hole molecules carrying charge not proportional to $\gamma_{0}$. We shall not need the explicit form of this contribution which is hard to compute in general when there are multiple other black hole molecules distributed in space, but use the fact that for a charged particle in a magnetic field the number of states per unit area in the lowest Landau level is proportional to the charge carried by the particle. To be more specific, we compare a configuration of a molecule of charge $s \gamma_{0}$ moving in the background of other molecules carrying arbitrary charges with a configuration of $s$ closeby molecules each of charge $\gamma_{0}$ moving in the same background. In this case the phase space volume element for the molecule of charge $s \gamma_{0}$ will be $s$ times the phase space volume element for each molecule of charge $\gamma_{0}$. Thus for a fixed background the orbital density of states for a black hole molecule carrying charge $s \gamma_{0}$, being proportional to the phase space volume element, will be $s$ times the orbital density of states of a molecule of charge $\gamma_{0}$. Thus we have $d\left(s \gamma_{0}\right) / d\left(\gamma_{0}\right)=s \Omega^{+}\left(s \gamma_{0}\right) / \Omega^{+}\left(\gamma_{0}\right)$, and hence ${ }^{5}$

$$
\bar{d}\left(s \gamma_{0}\right) / d\left(\gamma_{0}\right)=\sum_{m \mid s} m^{-1} d\left(s \gamma_{0} / m\right) / d\left(\gamma_{0}\right)=s \sum_{m \mid s} m^{-2} \Omega^{+}\left(s \gamma_{0} / m\right) / \Omega^{+}\left(\gamma_{0}\right)
$$

\footnotetext{
${ }^{4}$ At short distance they may interact via the exchange of massive string modes and also via dipole and higher order interactions due to massless particle exchange, but we do not expect these interactions to affect the analysis of supersymmetric index.

${ }^{5}$ Note that in this argument the sign of $d$, and hence the statistics of the particle, is determined by the sign of $\Omega^{+}$. Although the orbital angular momentum contributes to the index of the final configuration, they arise from the electromagnetic field, and hence do not affect the statistics of the individual particles in the halo.
} 
Comparing this with

$$
d\left(s \gamma_{0}\right) / d\left(\gamma_{0}\right)=s \Omega^{+}\left(s \gamma_{0}\right) / \Omega^{+}\left(\gamma_{0}\right)
$$

we see that replacing $d(\gamma)$ by $\bar{d}(\gamma)$ is equivalent to replacing $\Omega^{+}(\gamma)$ by

$$
\bar{\Omega}^{+}(\gamma)=\sum_{m \mid \gamma} m^{-2} \Omega^{+}(\gamma / m) .
$$

We shall see in $\S 4$ that the fractional DT invariants $\bar{\Omega}(\gamma)$ arise naturally in the KS wallcrossing formula.

We end this section with a word of caution. For a generic interacting system of bosons or fermions the effect of statistics and interaction cannot always be disentangled to map the problem to an equivalent problem with Boltzmann particles. Consider for example the case of an interacting system of two identical particles for which at a certain energy eigenvalue $E$ we have $n_{s}$ symmetric and $n_{a}$ anti-symmetric wave-functions. In this case we shall not get any simple map from the bosonic or fermionic system to a system of Boltzmann particles. Only if the identical particles are non-interacting so that multi-particle wave-functions can be constructed from (anti-)symmetric products of single particle wave functions, can we have a simple map from a Bose/Fermi gas to a Boltzmann gas.

\subsection{General wall-crossing formula and charge conservation}

The analysis of $\S 2.3$ leads to the following prescription for computing wall-crossing from supergravity black holes. Suppose in the chamber $c_{-}$we have a configuration of multicentered black hole molecules, consisting of $m_{r, s}$ centers of charge $\left(r \gamma_{1}+s \gamma_{2}\right)$ for different values of $(r, s)$. These molecules interact via long range electromagnetic, gravitational and other massless particle exchange interactions. We now consider a quantum mechanics of this system regarding the different centers as distinguishable particles, each with unit degeneracy, and denote by $g\left(\left\{m_{r, s}\right\}\right)$ trace of $(-1)^{2 J_{3}}$ in this quantum mechanics. Then the wall-crossing formula will be given by

$$
\begin{aligned}
\Delta \bar{\Omega}\left(M \gamma_{1}+N \gamma_{2}\right) & \equiv \bar{\Omega}^{-}\left(M \gamma_{1}+N \gamma_{2}\right)-\bar{\Omega}^{+}\left(M \gamma_{1}+N \gamma_{2}\right) \\
& =\sum_{\substack{\left\{m_{r, s}\right\} \\
\sum_{r, s} r m_{r, s}=M, \sum_{r, s} s m_{r, s}=N}} g\left(\left\{m_{r, s}\right\}\right) \prod_{r, s}\left[\frac{1}{\left(m_{r, s}\right) !}\left(\bar{\Omega}^{+}\left(r \gamma_{1}+s \gamma_{2}\right)\right)^{m_{r, s}}\right]
\end{aligned}
$$

For $\operatorname{gcd}(M, N)=1$ we have $\bar{\Omega}^{ \pm}\left(M \gamma_{1}+N \gamma_{2}\right)=\Omega^{ \pm}\left(M \gamma_{1}+N \gamma_{2}\right)$. Eq. (2.15) then follows from the fact that the left hand side represents the change in the index and the right hand side represents the total contribution from the bound states of black hole molecules which exist in the chamber $c_{-}$but not in the chamber $c_{+}$. For $\operatorname{gcd}(M, N)>1$ the indices $\Omega^{ \pm}\left(M \gamma_{1}+N \gamma_{2}\right)$ are somewhat ill defined since the total index in the sector of charge $\left(M \gamma_{1}+N \gamma_{2}\right)$ can receive contribution also from unbound multiparticle states carrying charges $\left(M \gamma_{1}+N \gamma_{2}\right) / s$ for integers $s \mid(M, N)$. Thus the unambiguous quantity is the effective index which enters the formula for the index of a bigger system of which the 
system with charge $\left(M \gamma_{1}+N \gamma_{2}\right)$ may form a subsystem [23]. This is what we call $\bar{\Omega}^{ \pm}$and this is the quantity whose jump is computed by the right hand side of (2.15).

A slightly different way of expressing (2.15) is:

$$
\bar{\Omega}^{-}(\gamma)-\bar{\Omega}^{+}(\gamma)=\sum_{n \geq 2} \sum_{\substack{\left\{\alpha_{1}, \ldots, \alpha_{n} \in \tilde{\Gamma}\right\} \\ \gamma=\alpha_{1}+\cdots+\alpha_{n}}} \frac{g\left(\left\{\alpha_{i}\right\}\right)}{\left|\operatorname{Aut}\left(\left\{\alpha_{i}\right\}\right)\right|} \prod_{i=1}^{n} \bar{\Omega}^{+}\left(\alpha_{i}\right),
$$

where the sum runs over all possible unordered decompositions of $\alpha$ into vectors $\alpha_{1}, \ldots, \alpha_{n} \in \tilde{\Gamma}$. The integer $\left|\operatorname{Aut}\left(\left\{\alpha_{i}\right\}\right)\right|$ is defined as follows. If all the $\alpha_{i}$ 's are distinct then $\left|\operatorname{Aut}\left(\left\{\alpha_{i}\right\}\right)\right|=1$. If on the other hand the set $\left(\alpha_{1}, \ldots, \alpha_{n}\right)$ consists of $m_{1}$ copies of a vector $\beta_{1}, m_{2}$ copies of a vector $\beta_{2}$ etc. then $\left|\operatorname{Aut}\left(\left\{\alpha_{i}\right\}\right)\right|=\prod_{a} m_{a}$ !. Finally $g\left(\alpha_{1}, \ldots, \alpha_{n}\right)$ represents the index associated with an $n$-centered black hole configuration in supergravity, with the individual centers carrying charges $\alpha_{1}, \alpha_{2}, \ldots, \alpha_{n}$ and treated as distinguishable particles carrying no internal degeneracy. Note that by an abuse of notation we have used for the argument of $g$ two different representations of the index of multi-centered black holes - one where the arguments are charges carried by individual centers and the other where the arguments are integers specifying how many of the centers carry a given charge vector $r \gamma_{1}+s \gamma_{2}$.

An immediate consequence of (2.16) is 'charge conservation' - the sum of the charges appearing in the arguments of $\bar{\Omega}^{+}$on the right hand side of the equation is equal to the argument of $\Delta \bar{\Omega}$ on the left hand side of this equation. In contrast if we had written the wall-crossing formula using the indices $\Omega^{+}$on the right hand side then there is no manifest charge conservation. This is a consequence of the fact that the use of $\bar{\Omega}$ allows us to use Maxwell-Boltzmann statistics for computing the contribution to the index due to multiple black hole molecules. In contrast if we had used Bose or Fermi statistics then manifest charge conservation is spoiled by the symmetrization effect since the degeneracy of $k$ identical particles carrying index $\Omega^{+}$not only contains a term proportional to $\left(\Omega^{+}\right)^{k}$ but also other terms containing lower powers of $\Omega^{+}$.

\subsection{Semi-primitive wall-crossing from Boltzmann gas of black hole molecules}

In this section we shall derive the semi-primitive wall-crossing formula by counting the index of a gas of black hole molecules carrying charges $s \gamma_{2}$ for different integers $s$, forming a halo around another black hole molecule of primitive charge $\gamma_{1}$. We denote by $k \gamma_{2}$ the total charge carried by the black hole gas. As noted in section $\S 2.3$, for this calculation we can regard the gas as one obeying Maxwell-Boltzmann statistics as long as we replace the index $\Omega^{+}\left(s \gamma_{2}\right)$ of a black hole molecule carrying charge $s \gamma_{2}$ by $\bar{\Omega}^{+}\left(s \gamma_{2}\right)$. The orbital motion of a black hole molecule of charge $s \gamma_{2}$ around a molecule of charge $\gamma_{1}$ produces states carrying angular momentum $\left(\left|\left\langle\gamma_{1}, s \gamma_{2}\right\rangle\right|-1\right) / 2$, and gives a contribution $(-1)^{\left\langle\gamma_{1}, s \gamma_{2}\right\rangle+1}\left|\left\langle\gamma_{1}, s \gamma_{2}\right\rangle\right|$ to the index [10]. Taking into account this additional factor we get the total contribution to the index from a single black hole molecule of charge $s \gamma_{2}$ to be

$$
(-1)^{\left\langle\gamma_{1}, s \gamma_{2}\right\rangle+1}\left|\left\langle\gamma_{1}, s \gamma_{2}\right\rangle\right| \bar{\Omega}^{+}\left(s \gamma_{2}\right) \text {. }
$$


Since we have already chosen $\left\langle\gamma_{1}, \gamma_{2}\right\rangle$ to be negative we can drop the absolute value sign and the +1 from the exponent. Thus if the halo consists of $m_{s}$ black hole molecules of charge $s \gamma_{2}$ then the net contribution to the index is

$$
\prod_{s}\left[\frac{1}{m_{s} !}\left((-1)^{\left\langle\gamma_{1}, s \gamma_{2}\right\rangle}\left\langle\gamma_{1}, s \gamma_{2}\right\rangle \bar{\Omega}^{+}\left(s \gamma_{2}\right)\right)^{m_{s}}\right] .
$$

Summing over all possible values of $m_{s}$ subject to the condition $\sum_{s} s m_{s}=N$, and multiplying this by the index $\Omega^{+}\left(\gamma_{1}\right)$ of the black hole molecule of charge $\gamma_{1}$ we get a total contribution $\Omega^{+}\left(\gamma_{1}\right) \Omega_{\text {halo }}\left(\gamma_{1}, N\right)$, where

$$
\Omega_{\text {halo }}\left(\gamma_{1}, N\right) \equiv \sum_{\substack{\left\{m_{s}\right\} \\ \sum_{s} s m_{s}=N}} \prod_{s}\left[\frac{1}{m_{s} !}\left((-1)^{\left\langle\gamma_{1}, s \gamma_{2}\right\rangle}\left\langle\gamma_{1}, s \gamma_{2}\right\rangle \bar{\Omega}^{+}\left(s \gamma_{2}\right)\right)^{m_{s}}\right] .
$$

This is the jump in the index due to a bound state of a black hole molecule of charge $\gamma_{1}$ and a halo of black hole molecules carrying charges $s \gamma_{2}$ for different integers $s$. In order to calculate the total change in the index in the sector of charge $\gamma_{1}+N \gamma_{2}$ across the wall of marginal stability $\mathcal{P}\left(\gamma_{1}, \gamma_{2}\right)$, we need to sum over all possible bound states containing a core of charge $\gamma_{1}+l \gamma_{2}$ and a halo of total charge $(N-l) \gamma_{2}$. Thus we have to sum over several terms of the form (2.19) with $\gamma_{1}$ replaced by $\gamma_{1}+l \gamma_{2}$ and $N$ replaced by $(N-l)$ for different integers $l$. This gives

$$
\Delta \bar{\Omega}\left(\gamma_{1}+N \gamma_{2}\right)=\sum_{l=0}^{N-1} \bar{\Omega}^{+}\left(\gamma_{1}+l \gamma_{2}\right) \Omega_{\text {halo }}\left(\gamma_{1}, N-l\right)
$$

where we have used the primitivity of $\gamma_{1}+\ell \gamma_{2}$ to replace $\Omega^{ \pm}\left(\gamma_{1}+\ell \gamma_{2}\right)$ by $\bar{\Omega}^{ \pm}\left(\gamma_{1}+\ell \gamma_{2}\right)$ for $0 \leq \ell \leq N$. This can be formalized in terms of the partition function

$$
\bar{Z}^{ \pm}(1, q)=\sum_{N=0}^{\infty} \bar{\Omega}^{ \pm}\left(\gamma_{1}+N \gamma_{2}\right) q^{N}
$$

Then $(2.20)$ can be written as

$$
\bar{Z}^{-}(1, q)=\bar{Z}^{+}(1, q) Z_{\text {halo }}\left(\gamma_{1}, q\right)
$$

where

$$
Z_{\text {halo }}\left(\gamma_{1}, q\right)=\sum_{N \geq 0} \Omega_{\text {halo }}\left(\gamma_{1}, N\right) q^{N}=\exp \left(\sum_{s=1}^{\infty} q^{s}(-1)^{\left\langle\gamma_{1}, s \gamma_{2}\right\rangle}\left\langle\gamma_{1}, s \gamma_{2}\right\rangle \bar{\Omega}^{+}\left(s \gamma_{2}\right)\right) .
$$

We shall see later that this agrees with the KS and JS wall-crossing formula restricted to the semi-primitive case (eqs.(4.29), (4.30) and (5.26)). For $N=1$ using $\gamma_{12}<0$, we recover the primitive wall-crossing formula (1.1).

To recover the semi-primitive wall-crossing formula of [10] from (2.22), (2.23), we proceed as follows. First of all we note that the relation (2.14) can be inverted as

$$
\Omega(\gamma)=\sum_{d \mid \gamma} \frac{1}{d^{2}} \mu(d) \bar{\Omega}(\gamma / d)
$$


where $\mu(d)$ is the Möbius function (i.e. 1 if $d$ is a product of an even number of distinct primes, -1 if $d$ is a product of an odd number of primes, or 0 otherwise). Using the identity $\prod_{d=1}^{\infty}\left(1-q^{d}\right)^{\mu(d) / d}=e^{-q}$ we can now express $(2.23)$ as

$$
Z_{\text {halo }}\left(\gamma_{1}, q\right)=\prod_{k>0}\left(1-(-1)^{k \gamma_{12}} q^{k}\right)^{k\left|\gamma_{12}\right| \Omega^{+}\left(k \gamma_{2}\right)}
$$

eqs.(2.22), (2.25) give precisely the semi-primitive wall-crossing formula of [10].

\subsection{Refined 'index' in supergravity}

Kontsevich and Soibelman also analyzed the wall crossing formula for the motivic DT invariants, which are quantized versions of the numerical DT invariants $\Omega(\gamma ; y)$. They enumerate the Betti numbers of the moduli space of BPS-states for given charge $\gamma$ in the weak string coupling regime, whereas $\Omega(\gamma)$ equals the Euler characteristics of this moduli space, up to a sign. Physically, the motivic DT invariants keep track of the angular momentum quantum numbers carried by the black hole at weak string coupling where the system may be represented as a collection of D6-D4-D2-D0 branes wrapped on a CalabiYau 3-fold [34, 35]. A simple way to do this is to introduce an extra factor of $y^{2 J_{3}}$ inside the trace in $(2.4)$ besides the $(-1)^{2 J_{3}}$ factor that is already present in this definition of the index. Thus at a given point in moduli space, the refined 'index' ${ }^{6} \Omega_{\text {ref }}(\gamma, y)$ is defined by $[11,34,35]$

$$
\Omega_{\mathrm{ref}}(\gamma, y)=\operatorname{Tr}_{\mathcal{H}(\gamma)}^{\prime}(-y)^{2 J_{3}} \equiv \sum_{n \in \mathbb{Z}}(-y)^{n} \Omega_{\mathrm{ref}, n}(\gamma)
$$

where $\operatorname{Tr}^{\prime}$ denotes the trace over BPS states computed after removing the contribution from the fermion zero modes associated with the supersymmetries broken by the black hole. Alternatively we could compute the ordinary trace over all the BPS states and then divide the result by $\left(2-y-y^{-1}\right)$ which represents the contribution from the fermion zero modes. The usual generalized DT invariants are obtained by setting $y=1$,

$$
\Omega(\gamma) \equiv \sum_{n \in \mathbb{Z}}(-1)^{n} \Omega_{\mathrm{ref}, n}(\gamma)=\Omega_{\mathrm{ref}}(\gamma, y=1)
$$

In string theory (2.26) is not an index since it is not protected against quantum corrections as we switch on the string coupling. In supersymmetric gauge theories one can define an alternative version of this index as [22],

$$
\Omega_{\mathrm{ref}}^{\prime}(\gamma, y)=\operatorname{Tr}_{H(\gamma)}\left(2 J_{3}\right)(-1)^{2 J_{3}}(-y)^{2 I_{3}+2 J_{3}} \equiv \sum_{n \in \mathbb{Z}}(-y)^{n} \Omega_{\mathrm{ref}, n}^{\prime}(\gamma)
$$

where $I_{3}$ is the third component of the $\mathrm{SU}(2)_{R}$ symmetry. This is protected against quantum corrections. We shall however proceed with the definition (2.26) since our main interest is in string theory. Even though there is no general argument that protects this 'index' from changing as we vary the string coupling, and hence the DT invariants and the black hole degeneracies may not be equal, we may nevertheless expect that the structure of the

\footnotetext{
${ }^{6}$ We shall use the words motivic and refined interchangeably.
} 
wall-crossing formula at fixed coupling will remain the same. Thus we can regard the motivic KS formula as giving the change in $\Omega_{\text {ref }}(\gamma, y)$ across a wall of marginal stability at fixed value of the string coupling. With this in mind we shall analyze the jump in the motivic 'index' in supergravity and then compare this with the KS formula. Our supergravity analysis, compared to that in $\S 2.3$, will be somewhat heuristic; however the final result of this analysis will turn out to be consistent with the KS motivic wall-crossing formula.

We begin by introducing some notations. We have already denoted by $\Omega_{\mathrm{ref}}(\gamma, y)$ the refined 'index' computed by introducing a weight factor of $y^{2 J_{3}}$ into the trace in (2.4). More generally we shall use the subscript ref to denote various quantities in which the trace over different angular momentum states has been performed with a weight factor of $y^{2 J_{3}}$. With this the analog of (2.10) takes the form

$$
\bar{d}\left(\gamma, J_{3}\right)=\sum_{m \mid \gamma, 2 J_{3}} m^{-1} d\left(\gamma / m, J_{3} / m\right) .
$$

A word of caution is warranted here. Since the full system is rotationally invariant, the states of this system can be characterized by their angular momentum. However when we examine the motion of one subsystem in the background of the other, the background generically breaks rotational invariance and hence states can no longer be classified by their angular momentum unless the background is generated by a point source (or a spherically symmetric source). We shall nevertheless proceed as if each subsystem consisting of a set of identical particles moved in the background produced by a point source so that an assignment of angular momentum quantum numbers to such individual subsystems were possible. Based on this assumption we shall arrive at an expression for the motivic index of the whole system in terms of the index carried by the individual molecules. This procedure can be justified a posteriori by the fact that it allows for a physical understanding of the motivic generalization of the KS wall-crossing formula.

After multiplying $(2.29)$ by $y^{2 J_{3}}$ and summing over $J_{3}$ we get

$$
\begin{aligned}
\bar{d}_{\mathrm{ref}}(\gamma, y) & =\sum_{J_{3}} \sum_{m \mid \gamma, 2 J_{3}} m^{-1} d\left(\gamma / m, J_{3} / m\right) y^{2 J_{3}}=\sum_{m \mid \gamma} \sum_{J_{3}^{\prime}} m^{-1} d\left(\gamma / m, J_{3}^{\prime}\right) y^{2 m J_{3}^{\prime}} \\
& =\sum_{m \mid \gamma} m^{-1} d_{\mathrm{ref}}\left(\gamma / m, y^{m}\right) .
\end{aligned}
$$

Our next task is to find the generalization of (2.12). Let us denote by $d_{\text {orb }}\left(\gamma, J_{3}\right)$ the degeneracy due to orbital motion of a black hole molecule of charge $\gamma$ in some fixed background. Again we pretend that the background is spherically symmetric so that it makes sense to assign definite angular momentum quantum numbers to the orbital states of individual subsystems. Then we have

$$
d_{\text {ref }}(\gamma, y)=\Omega_{\text {ref }}^{+}(\gamma, y) d_{\text {orb;ref }}(\gamma, y)
$$

where

$$
d_{\mathrm{orb} ; \mathrm{ref}}(\gamma, y)=\sum_{J_{3}} d_{\mathrm{orb}}\left(\gamma, J_{3}\right) y^{2 J_{3}}
$$


eq. (2.30) and (2.31) now give

$$
\bar{d}_{\mathrm{ref}}(\gamma, y)=\sum_{m \mid \gamma} m^{-1} \Omega_{\mathrm{ref}}^{+}\left(\gamma / m, y^{m}\right) d_{\mathrm{orb} ; \mathrm{ref}}\left(\gamma / m, y^{m}\right) .
$$

We shall now try to express $d_{\text {orb;ref }}\left(\gamma / m, y^{m}\right)$ in terms of $d_{\text {orb;ref }}(\gamma, y)$. For this (still pretending that we have a rotationally invariant subsystem) we shall decompose the orbital spectrum into $\mathrm{SU}(2)$ representations and denote by $b(\gamma, J)$ the coefficient of the character of the representation of $\operatorname{spin} J$. Then we have

$$
d_{\text {orb;ref }}(\gamma, y)=\sum_{J} b(\gamma, J)\left(y^{2 J}+y^{2 J-2}+\cdots+y^{-2 J}\right)=\sum_{J} b(\gamma, J) \frac{y^{2 J+1}-y^{-2 J-1}}{y-y^{-1}} .
$$

We now use the fact that for any $m \in \mathbb{Z}^{+}$, and for spherically symmetric background, we have

$$
b(\gamma, J)=b\left(m \gamma, J^{\prime}\right), \quad 2 J^{\prime}+1=m(2 J+1) .
$$

Effectively (2.35) follows from the fact that increasing the charge of the molecule by a factor of $m$ changes the angular momentum carried by the lowest Landau level such that the degeneracy of the Landau level gets scaled by a factor of $m$. Using this we get

$$
d_{\text {orb;ref }}\left(\gamma / m, y^{m}\right)=\sum_{J^{\prime}} b\left(\gamma, J^{\prime}\right) \frac{y^{\left(2 J^{\prime}+1\right)}-y^{-\left(2 J^{\prime}+1\right)}}{y^{m}-y^{-m}}=\frac{y-y^{-1}}{y^{m}-y^{-m}} d_{\text {orb;ref }}(\gamma, y) \text {. }
$$

Substituting this into (2.33) we arrive at

$$
\bar{d}_{\mathrm{ref}}(\gamma, y)=\bar{\Omega}_{\mathrm{ref}}^{+}(\gamma, y) d_{\mathrm{orb} ; \mathrm{ref}}(\gamma, y),
$$

where the "rational motivic invariants" $\bar{\Omega}_{\text {ref }}$ are defined by

$$
\bar{\Omega}_{\mathrm{ref}}(\gamma, y) \equiv \sum_{m \mid \gamma} \frac{y-y^{-1}}{m\left(y^{m}-y^{-m}\right)} \Omega_{\mathrm{ref}}\left(\gamma / m, y^{m}\right) .
$$

This shows that in computing the refined index of the full system we can treat the particles as obeying Maxwell-Boltzmann statistics provided we replace $\Omega_{\text {ref }}$ by $\bar{\Omega}_{\text {ref }}$. As in the case of the classical DT invariants, the use of these invariants ensures that only charge preserving terms appear in any wall-crossing formula.

The rational motivic invariants have appeared earlier in other contexts, for example in the construction of modular invariant partition functions in [30]. We would also like to point out that their structure is very similar to the free energy which arises in the computations by Gopakumar and Vafa [28]. The only difference is that in the Euclidean setup of [28], the factor $\left(y^{d}-y^{-d}\right)$ on the right-hand side of $(2.38)$ is replaced by $\left(y^{d}-y^{-d}\right)^{2}$. As in this case, the generating function of the rational invariants $\bar{\Omega}_{\text {ref }}$ leads to a product formula

$$
\sum_{\gamma} \frac{\bar{\Omega}_{\mathrm{ref}}(\gamma, y)}{y-y^{-1}} e^{-\gamma \cdot \phi}=\log \left[\prod_{\substack{n \in \mathbb{Z}, \ell \geq 0 \\ \gamma}}\left(1-y^{1+n+2 \ell} e^{-\gamma \cdot \phi}\right)^{(-1)^{n} \Omega_{\mathrm{ref}, n}(\gamma)}\right],
$$


where $\phi$ is a vector of chemical potentials conjugate to the charge vector $\gamma$. Note that this product structure is lost in the limit $y \rightarrow 1$.

The analog of (2.15) now takes the form ${ }^{7}$

$$
\begin{aligned}
& \Delta \bar{\Omega}_{\mathrm{ref}}\left(M \gamma_{1}+N \gamma_{2}, y\right) \equiv \bar{\Omega}_{\mathrm{ref}}^{-}\left(M \gamma_{1}+N \gamma_{2}, y\right)-\bar{\Omega}_{\mathrm{ref}}^{+}\left(M \gamma_{1}+N \gamma_{2}, y\right) \\
& =\sum_{\substack{\left\{m_{r, s}\right\} \\
\sum_{r, s} r m_{r, s}=M, \sum_{r, s} s m_{r, s}=N}} g_{\mathrm{ref}}\left(\left\{m_{r, s}\right\}, y\right) \prod_{r, s}\left[\frac{1}{\left(m_{r, s}\right) !}\left(\bar{\Omega}_{\mathrm{ref}}^{+}\left(r \gamma_{1}+s \gamma_{2}, y\right)\right)^{m_{r, s}}\right],
\end{aligned}
$$

where $g_{\mathrm{ref}}\left(\left\{m_{r, s}\right\}, y\right)$ measures $\operatorname{Tr}(-y)^{2 J_{3}}$ from orbital motion of a set of distinguishable particles, containing $m_{r, s}$ number of particles carrying charges $r \gamma_{1}+s \gamma_{2}$. Similarly the analog of (2.16) is

$$
\bar{\Omega}^{-}(\gamma, y)-\bar{\Omega}^{+}(\gamma, y)=\sum_{n \geq 2} \sum_{\substack{\left\{\alpha_{1}, \ldots, \alpha_{n} \in \tilde{\Gamma}\right\} \\ \gamma=\alpha_{1}+\cdots+\alpha_{n}}} \frac{g_{\mathrm{ref}}\left(\left\{\alpha_{i}\right\}, y\right)}{\left|\operatorname{Aut}\left(\left\{\alpha_{i}\right\}\right)\right|} \prod_{i=1}^{n} \bar{\Omega}_{\mathrm{ref}}^{+}\left(\alpha_{i}, y\right),
$$

consistently with the charge conservation property of the motivic wall crossing formula when expressed in terms of the combinations $\bar{\Omega}_{\text {ref }}^{ \pm}$. Note that even though our derivation of (2.41) has been marred by unreasonable assumption of spherical symmetry in the dynamics of various subsystems, each term in (2.41) is defined unambiguously so that it can be put to test against known results.

We can also easily derive the semi-primitive version of the motivic wall-crossing formula by following the logic of $\S 2.5$. It takes the form ${ }^{8}$

$$
\begin{aligned}
& \Delta \Omega_{\mathrm{ref}}\left(\gamma_{1}+N \gamma_{2}, y\right)= \\
& \sum_{l=0}^{N-1} \bar{\Omega}_{\mathrm{ref}}^{+}\left(\gamma_{1}+l \gamma_{2}, y\right) \sum_{\substack{\left\{m_{s}\right\} \\
\sum_{s} s m_{s}=N-l}} \prod_{s}\left[\frac{1}{m_{s} !}\left(\left(\frac{(-y)^{\left\langle\gamma_{1}, s \gamma_{2}\right\rangle}-(-y)^{-\left\langle\gamma_{1}, s \gamma_{2}\right\rangle}}{y-y^{-1}}\right) \bar{\Omega}_{\mathrm{ref}}^{+}\left(s \gamma_{2}, y\right)\right)^{m_{s}}\right] .
\end{aligned}
$$

We shall see later that this is in perfect agreement with the prediction of KS motivic wall-crossing formula (4.72).

\section{Multi-black hole bound states and quiver quantum mechanics}

In order to have a complete wall-crossing formula we need to find explicit expressions for the functions $g\left(\left\{\alpha_{i}\right\}\right), g_{\mathrm{ref}}\left(\left\{\alpha_{i}\right\}, y\right)$ appearing in eqs.(2.16) and (2.41) respectively. This

\footnotetext{
${ }^{7}$ Since a single centered BPS black hole is expected to carry zero angular momentum [47-49], one might naively expect $\bar{\Omega}_{\text {ref }}^{+}(\gamma, y)$ to be independent of $y$. However as discussed in $\S 2.2$, we allow for centers which consist of multi-centered black holes whose relative separation remains finite as we approach the wall of marginal stability. As a result $\Omega_{\mathrm{ref}}(\gamma, y)$ can be a non-trivial function of $y$.

${ }^{8}$ Note that in the semi-primitive case our heuristic derivation becomes rigorous since identical particles carrying charge $s \gamma_{2}$ move in the spherically symmetric background produced by the charge $\gamma_{1}+l \gamma_{2}$. Since [23] argues that general wall-crossing formula can be derived from the semi-primitive formula, we can use this to justify our general claim (2.41).
} 
requires finding the spectrum of supersymmetric bound states of multi-black hole configurations in supergravity. As argued by Denef [7], the supersymmetric quantum mechanics of multi-centered BPS configurations can be viewed as the "Coulomb phase" of a quiver matrix model, valid at strong coupling. At weak coupling, the wave function is instead supported on the Higgs branch. It should be possible to compute the function $g\left(\left\{\alpha_{i}\right\}\right)$, $g_{\text {ref }}\left(\left\{\alpha_{i}\right\}, y\right)$ from either description. In this section we shall describe both these approaches. As we shall see, the description on the Higgs branch, described in $\S 3.1$, allows us to solve the problem completely. On the other hand the description on the Coulomb branch, described in $\S 3.2$, also gives a complete algorithm for finding $g\left(\left\{\alpha_{i}\right\}\right)$, but it is more difficult to solve it explicitly. Furthermore with some guesswork we can also arrive at a specific proposal for $g_{\text {ref }}\left(\left\{\alpha_{i}\right\}, y\right)$ from the analysis on the Coulomb branch.

Without any loss of generality we can arrange the $\alpha_{i}$ 's so that

$$
\alpha_{i j} \equiv\left\langle\alpha_{i}, \alpha_{j}\right\rangle \geq 0 \quad \text { for } \quad i<j
$$

Now if we represent a vector $M \gamma_{1}+N \gamma_{2}$ in $\tilde{\Gamma}$ by the point $(M, N)$ in the Cartesian coordinate system, then in this representation a pair of vectors $(\alpha, \beta)$ will follow clockwise (anti-clockwise) order if $\langle\alpha, \beta\rangle$ is positive (negative). The condition (3.1) then implies that the vectors $\alpha_{1}, \cdots \alpha_{n}$ are arranged in a clockwise fashion. Throughout this section we shall work with this particular ordering of the $\alpha_{i}$ 's.

\subsection{Higgs branch analysis}

As has been argued by Denef [8], the bound state spectrum of multi-centered black holes can also be computed using quiver quantum mechanics. For computing $g_{\text {ref }}\left(\left\{\alpha_{i}\right\}, y\right)$ we need to study the bound states of $n$ distinguishable particles. In this case the quiver takes a simple form with $n$-nodes each carrying a U(1) factor, and $\alpha_{i j}$ arrows from the node $i$ to the node $j$ for $i<j$. In particular since the arrows always go from lower to higher node, there are no oriented loops.

Now for quivers without oriented loops, Reineke [40] has computed the generating function of the Betti numbers of the space of semi-stable solutions to the D-flatness conditions. Physically they determine the number of supersymmetric quantum states carrying given angular momentum $J_{3}$. The formula takes a simple form when all nodes carry $\mathrm{U}(1)$ factors and we shall state the formula for this special case. According to this formula $\operatorname{Tr}\left((-y)^{2 J_{3}}\right)$, which can be identified with the function $g_{\mathrm{ref}}\left(\left\{\alpha_{i}\right\}, y\right)$, is given by

$$
g_{\mathrm{ref}}\left(\alpha_{1}, \ldots, \alpha_{n}, y\right)=(-y)^{-L}\left(y^{2}-1\right)^{1-n} \sum_{\text {partitions }}(-1)^{s-1} y^{2 \sum_{a \leq b} \sum_{j<i} \alpha_{j i} m_{i}^{(a)} m_{j}^{(b)}}
$$

where the sum runs over all ordered partitions of $\left(\alpha_{1}+\cdots+\alpha_{n}\right)$ into $s$ vectors $\beta^{(a)}$ $(1 \leq a \leq s, 1 \leq s \leq n)$ such that

1. $\sum_{a} \beta^{(a)}=\alpha_{1}+\cdots+\alpha_{n}$.

2. $\beta^{(a)}=\sum_{i} m_{i}^{(a)} \alpha_{i}$ with $m_{i}^{(a)}=0$ or 1 for each $a, i$. 
3. $\arg \left(\sum_{a=1}^{b} Z_{\beta^{(a)}}\right)>\arg \left(Z_{\alpha_{1}+\cdots+\alpha_{n}}\right)$ for all $b$ between 1 and $s-1$ in the chamber $c^{-}$.

Using the fact that in the chamber $c^{-} \arg \left(Z_{\alpha}\right)>\arg \left(Z_{\beta}\right)$ implies $\langle\alpha, \beta\rangle>0$, we can express condition 3. as

$$
\left\langle\sum_{a=1}^{b} \beta^{(a)}, \alpha_{1}+\cdots+\alpha_{n}\right\rangle>0 \quad \forall \quad b \quad \text { with } \quad 1 \leq b \leq s-1 .
$$

In (3.2) $L$ is a constant given by

$$
L=(1-n)+\sum_{i<j} \alpha_{i j}
$$

in such a way that (3.2) is invariant under $y \rightarrow y^{-1}$. Physically $L$ represents the maximum $2 J_{3}$ eigenvalue that the system can carry.

We shall now illustrate how this formula works by computing $g_{\mathrm{ref}}\left(\left\{\alpha_{i}\right\}, y\right)$ for $n=2,3$ and 4. First consider the $n=2$ case with $\alpha_{12}>0$. In this case we have two possible ordered partitions satisfying the conditions stated above:

$$
\left\{\alpha_{1}+\alpha_{2}\right\}, \quad\left\{\alpha_{1}, \alpha_{2}\right\}
$$

The first term contributes $y^{2 \alpha_{12}}$ and the second term contributes -1 to the sum. In total,

$$
g_{\mathrm{ref}}\left(\alpha_{1}, \alpha_{2}, y\right)=(-y)^{1-\alpha_{12}}\left(y^{2}-1\right)^{-1}\left(y^{2 \alpha_{12}}-1\right)=(-1)^{\alpha_{12}+1} \frac{y^{\alpha_{12}}-y^{-\alpha_{12}}}{y-y^{-1}} .
$$

This correctly produces the spectrum of two centered bound states. Taking the $y \rightarrow 1$ limit gives

$$
g\left(\alpha_{1}, \alpha_{2}\right)=(-1)^{\alpha_{12}+1} \alpha_{12}
$$

Let us now turn to the $n=3$ case. We take $\alpha_{1}, \alpha_{2}, \alpha_{3}$ to be three distinct elements (not necessarily primitive) of $\tilde{\Gamma}$ such that $\alpha_{12}, \alpha_{13}$ and $\alpha_{23}$ are all positive. For definiteness we shall choose the $\alpha_{i}$ 's such that $\alpha_{12}>\alpha_{23}$. In the convention described above (3.1), the charges listed below follow a clockwise order as we move from left to right:

$$
\alpha_{1}, \quad\left(\alpha_{1}+\alpha_{2}, \quad \alpha_{1}+\alpha_{3}\right), \quad \alpha_{1}+\alpha_{2}+\alpha_{3}, \quad \alpha_{2}, \quad \alpha_{2}+\alpha_{3}, \quad \alpha_{3} .
$$

The relative ordering of the vectors inside ( ) is not determined unambiguously but is unimportant. The condition (3.3) is now easy to implement: for every $b, \sum_{a=1}^{b} \beta^{(b)}$ must be one of the vectors to the left of $\alpha_{1}+\alpha_{2}+\alpha_{3}$ in the list (3.8). In this case the possible ordered partitions of $\alpha_{1}+\alpha_{2}+\alpha_{3}$ satisfying (3.3) are:

$\left\{\alpha_{1}+\alpha_{2}+\alpha_{3}\right\},\left\{\alpha_{1}, \alpha_{2}+\alpha_{3}\right\},\left\{\alpha_{1}+\alpha_{2}, \alpha_{3}\right\},\left\{\alpha_{1}+\alpha_{3}, \alpha_{2}\right\},\left\{\alpha_{1}, \alpha_{2}, \alpha_{3}\right\},\left\{\alpha_{1}, \alpha_{3}, \alpha_{2}\right\}$. 
This gives, after a cancelation between the second and the last contribution,

$$
\begin{aligned}
g_{\mathrm{ref}}\left(\alpha_{1}, \alpha_{2}, \alpha_{3}, y\right)= & (-1)^{\alpha_{12}+\alpha_{13}+\alpha_{23}}\left(y-y^{-1}\right)^{-2} \\
& \left(y^{\alpha_{12}+\alpha_{13}+\alpha_{23}}-y^{\alpha_{12}-\alpha_{23}-\alpha_{13}}-y^{\alpha_{13}+\alpha_{23}-\alpha_{12}}+y^{-\alpha_{12}-\alpha_{13}-\alpha_{23}}\right) \\
= & (-1)^{\alpha_{12}+\alpha_{13}+\alpha_{23}} \frac{1}{\sinh ^{2} \nu} \sinh \left(\nu \alpha_{12}\right) \sinh \left(\nu\left(\alpha_{13}+\alpha_{23}\right)\right),
\end{aligned}
$$

where $\nu \equiv \ln y$. Taking the $y \rightarrow 1$ limit we get

$$
g\left(\alpha_{1}, \alpha_{2}, \alpha_{3}\right)=(-1)^{\alpha_{12}+\alpha_{13}+\alpha_{23}} \alpha_{12}\left(\alpha_{13}+\alpha_{23}\right) .
$$

If instead $\alpha_{12}<\alpha_{23}$, a similar reasoning leads to

$$
\begin{aligned}
g_{\mathrm{ref}}\left(\alpha_{1}, \alpha_{2}, \alpha_{3}, y\right) & =(-1)^{\alpha_{12}+\alpha_{13}+\alpha_{23}} \frac{1}{\sinh ^{2} \nu} \sinh \left(\nu \alpha_{23}\right) \sinh \left(\nu\left(\alpha_{12}+\alpha_{13}\right)\right), \\
& \stackrel{y \rightarrow 1}{\rightarrow}(-1)^{\alpha_{12}+\alpha_{13}+\alpha_{23}} \alpha_{23}\left(\alpha_{12}+\alpha_{13}\right) .
\end{aligned}
$$

Next we consider the case $n=4$. We choose 4 vectors $\alpha_{1}, \alpha_{2}, \alpha_{3}, \alpha_{4}$ such that in the convention described below (3.1), different linear combinations of the $\alpha_{i}$ 's follow the following clockwise order as we move from left to right in the list:

$$
\begin{array}{r}
\alpha_{1},\left(\alpha_{1}+\alpha_{2}, \alpha_{1}+\alpha_{3}, \alpha_{1}+\alpha_{2}+\alpha_{3}\right), \alpha_{2},\left(\alpha_{2}+\alpha_{3}, \alpha_{1}+\alpha_{2}+\alpha_{4}\right), \\
\alpha_{1}+\alpha_{2}+\alpha_{3}+\alpha_{4}, \alpha_{3}, \alpha_{1}+\alpha_{3}+\alpha_{4},\left(\alpha_{1}+\alpha_{4}, \alpha_{2}+\alpha_{3}+\alpha_{4}, \alpha_{2}+\alpha_{4}, \alpha_{3}+\alpha_{4}\right), \alpha_{4},
\end{array}
$$

where again, the order of terms between brackets is irrelevant. We list below the allowed partitions consistent with the three conditions described above and the corresponding contribution to the summand:

$$
\begin{array}{|l|r|}
\hline\left\{\alpha_{1}+\alpha_{2}+\alpha_{3}+\alpha_{4}\right\} & y^{2\left(\alpha_{12}+\alpha_{13}+\alpha_{14}+\alpha_{23}+\alpha_{24}+\alpha_{34}\right)} \\
\left\{\alpha_{1}, \alpha_{2}+\alpha_{3}+\alpha_{4}\right\} & -y^{2\left(\alpha_{23}+\alpha_{24}+\alpha_{34}\right)} \\
\left\{\alpha_{2}, \alpha_{1}+\alpha_{3}+\alpha_{4}\right\} & -y^{2\left(\alpha_{12}+\alpha_{13}+\alpha_{14}+\alpha_{34}\right)} \\
\left\{\alpha_{1}+\alpha_{2}, \alpha_{3}+\alpha_{4}\right\} & -y^{2\left(\alpha_{12}+\alpha_{34}\right)} \\
\left\{\alpha_{1}+\alpha_{3}, \alpha_{2}+\alpha_{4}\right\} & -y^{2\left(\alpha_{13}+\alpha_{23}+\alpha_{24}\right)} \\
\left\{\alpha_{1}+\alpha_{2}+\alpha_{3}, \alpha_{4}\right\} & -y^{2\left(\alpha_{12}+\alpha_{13}+\alpha_{23}\right)} \\
\left\{\alpha_{2}+\alpha_{3}, \alpha_{1}+\alpha_{4}\right\} & -y^{2\left(\alpha_{12}+\alpha_{13}+\alpha_{14}+\alpha_{23}\right)} \\
\left\{\alpha_{1}+\alpha_{2}+\alpha_{4}, \alpha_{3}\right\} & -y^{2\left(\alpha_{12}+\alpha_{14}+\alpha_{24}+\alpha_{34}\right)} \\
\left\{\alpha_{1}, \alpha_{2}, \alpha_{3}+\alpha_{4}\right\} & y^{2 \alpha_{34}} \\
\left\{\alpha_{1}, \alpha_{2}+\alpha_{3}, \alpha_{4}\right\} & y^{2 \alpha_{23}} \\
\left\{\alpha_{1}+\alpha_{3}, \alpha_{2}, \alpha_{4}\right\} & y^{2\left(\alpha_{13}+\alpha_{23}\right)} \\
\left\{\alpha_{1}, \alpha_{3}, \alpha_{2}+\alpha_{4}\right\} & y^{2\left(\alpha_{23}+\alpha_{24}\right)} \\
\left\{\alpha_{2}, \alpha_{3}, \alpha_{1}+\alpha_{4}\right\} & y^{2\left(\alpha_{12}+\alpha_{13}+\alpha_{14}\right)} \\
\hline
\end{array}
$$

\begin{tabular}{|l|r|}
$\left\{\alpha_{1}, \alpha_{2}+\alpha_{4}, \alpha_{3}\right\}$ & $y^{2\left(\alpha_{24}+\alpha_{34}\right)}$ \\
$\left\{\alpha_{2}, \alpha_{1}, \alpha_{3}+\alpha_{4}\right\}$ & $y^{2\left(\alpha_{12}+\alpha_{34}\right)}$ \\
$\left\{\alpha_{2}, \alpha_{1}+\alpha_{3}, \alpha_{4}\right\}$ & $y^{2\left(\alpha_{12}+\alpha_{13}\right)}$ \\
$\left\{\alpha_{2}, \alpha_{1}+\alpha_{4}, \alpha_{3}\right\}$ & $y^{2\left(\alpha_{12}+\alpha_{14}+\alpha_{34}\right)}$ \\
$\left\{\alpha_{1}+\alpha_{2}, \alpha_{3}, \alpha_{4}\right\}$ & $y^{2 \alpha_{12}}$ \\
$\left\{\alpha_{1}+\alpha_{2}, \alpha_{4}, \alpha_{3}\right\}$ & $y^{2\left(\alpha_{12}+\alpha_{34}\right)}$ \\
$\left\{\alpha_{2}+\alpha_{3}, \alpha_{1}, \alpha_{4}\right\}$ & $y^{2\left(\alpha_{12}+\alpha_{13}+\alpha_{23}\right)}$ \\
$\left\{\alpha_{1}, \alpha_{2}, \alpha_{3}, \alpha_{4}\right\}$ & -1 \\
$\left\{\alpha_{1}, \alpha_{2}, \alpha_{4}, \alpha_{3}\right\}$ & $-y^{2 \alpha_{34}}$ \\
$\left\{\alpha_{1}, \alpha_{3}, \alpha_{2}, \alpha_{4}\right\}$ & $-y^{2 \alpha_{23}}$ \\
$\left\{\alpha_{2}, \alpha_{1}, \alpha_{3}, \alpha_{4}\right\}$ & $-y^{2 \alpha_{12}}$ \\
$\left\{\alpha_{2}, \alpha_{3}, \alpha_{1}, \alpha_{4}\right\}$ & $-y^{2\left(\alpha_{12}+\alpha_{13}\right)}$ \\
$\left\{\alpha_{2}, \alpha_{1}, \alpha_{4}, \alpha_{3}\right\}$ & $-y^{2\left(\alpha_{12}+\alpha_{34}\right)}$ \\
\hline
\end{tabular}


Adding these terms and substituting into eq.(3.2) we arrive at

$$
\begin{aligned}
& g_{\mathrm{ref}}\left(\alpha_{1}, \alpha_{2}, \alpha_{3}, \alpha_{4}, y\right) \\
= & (-1)^{\alpha_{12}+\alpha_{13}+\alpha_{14}+\alpha_{23}+\alpha_{24}+\alpha_{34}+1} \frac{1}{\left(y-y^{-1}\right)^{3}} \\
& \times\left(y^{\alpha_{12}+\alpha_{13}+\alpha_{14}-\alpha_{23}-\alpha_{24}-\alpha_{34}}-y^{\alpha_{12}+\alpha_{13}+\alpha_{14}+\alpha_{23}-\alpha_{24}-\alpha_{34}}+y^{\alpha_{12}-\alpha_{13}+\alpha_{14}-\alpha_{23}-\alpha_{24}+\alpha_{34}}\right. \\
& -y^{\alpha_{12}+\alpha_{13}+\alpha_{14}-\alpha_{23}-\alpha_{24}+\alpha_{34}}-y^{\alpha_{12}-\alpha_{13}+\alpha_{14}-\alpha_{23}+\alpha_{24}+\alpha_{34}}+y^{\alpha_{12}+\alpha_{13}+\alpha_{14}+\alpha_{23}+\alpha_{24}+\alpha_{34}} \\
& -y^{-\alpha_{12}-\alpha_{13}-\alpha_{14}-\alpha_{23}-\alpha_{24}-\alpha_{34}}+y^{-\alpha_{12}+\alpha_{13}-\alpha_{14}+\alpha_{23}-\alpha_{24}-\alpha_{34}}+y^{-\alpha_{12}-\alpha_{13}-\alpha_{14}+\alpha_{23}+\alpha_{24}-\alpha_{34}} \\
& \left.-y^{-\alpha_{12}+\alpha_{13}-\alpha_{14}+\alpha_{23}+\alpha_{24}-\alpha_{34}}+y^{-\alpha_{12}-\alpha_{13}-\alpha_{14}-\alpha_{23}+\alpha_{24}+\alpha_{34}}-y^{-\alpha_{12}-\alpha_{13}-\alpha_{14}+\alpha_{23}+\alpha_{24}+\alpha_{34}}\right) \\
= & (-1)^{\alpha_{12}+\alpha_{13}+\alpha_{14}+\alpha_{23}+\alpha_{24}+\alpha_{34}+1} \frac{1}{\sinh ^{3} \nu} \\
& {\left[\sinh \left(\nu \alpha_{13}\right) \sinh \left(\nu\left(-\alpha_{12}+\alpha_{23}+\alpha_{24}\right)\right) \sinh \left(\nu\left(\alpha_{14}+\alpha_{34}\right)\right)\right.} \\
& +\sinh \left(\nu \alpha_{14}\right) \sinh \left(\nu \alpha_{23}\right) \sinh \left(\nu\left(-\alpha_{12}-\alpha_{13}+\alpha_{24}+\alpha_{34}\right)\right) \\
& \left.+\sinh \left(\nu \alpha_{12}\right) \sinh \left(\nu\left(\alpha_{13}+\alpha_{23}\right)\right) \sinh \left(\nu\left(\alpha_{14}+\alpha_{24}+\alpha_{34}\right)\right)\right] .
\end{aligned}
$$

Taking the $y \rightarrow 1$ limit we get

$$
\begin{aligned}
g\left(\alpha_{1}, \alpha_{2}, \alpha_{3}, \alpha_{4}\right)= & (-1)^{1+\sum_{i<j} \alpha_{i j} \times} \\
& {\left[\alpha_{12} \alpha_{13} \alpha_{24}+\alpha_{13} \alpha_{14} \alpha_{24}+\alpha_{12} \alpha_{23} \alpha_{24}+\alpha_{14} \alpha_{23} \alpha_{24}\right.} \\
& \left.+\alpha_{12} \alpha_{23} \alpha_{34}+\alpha_{13} \alpha_{23} \alpha_{34}+\alpha_{14} \alpha_{23} \alpha_{34}+\alpha_{13} \alpha_{24} \alpha_{34}\right] .
\end{aligned}
$$

We have also derived the analog of (3.14) for $n=5$ but we suppress the result for the sake of brevity. Similar results can be obtained for different choices of orderings, while cases where some of the final states coincide can be obtained by taking suitable limits. For example consider the case $g_{\text {ref }}\left(\alpha_{1}, \alpha_{2}, \alpha_{2}, y\right)$ with $\alpha_{12}>0$. This can be considered as a special case of (3.10) in the limit $\alpha_{3} \rightarrow \alpha_{2}$ and gives

$$
g_{\mathrm{ref}}\left(\alpha_{1}, \alpha_{2}, \alpha_{2}\right)=\frac{1}{\sinh ^{2} \nu} \sinh \left(\nu \alpha_{12}\right) \sinh \left(2 \nu \alpha_{12}\right) .
$$

We shall later verify that the various explicit results given in this section are in perfect agreement with both KS and JS wall-crossing formulæ.

Thus, eq. (3.2) provides a complete algorithm for computing the coefficient $g_{\mathrm{ref}}\left(\left\{\alpha_{i}\right\}, y\right)$ in (2.41) for any number of $\alpha_{i}$ 's. This result is based on the study of multi-centered bound states in supergravity, even though we had to rely on mathematical results about moduli spaces of quiver representations. The key point is that we only needed invariants of Abelian quivers to compute the dynamics of internal (or hair) degrees of freedom, while each center could still be regarded as a macroscopic solution (provided its charge is large enough). In the next subsection, we relinquish the gauge theoretical crutch provided by Reineke's formula and directly quantize the internal degrees of freedom of the bound state. 


\subsection{Coulomb branch analysis}

In this subsection we shall try to reproduce the results of $\S 3.1$ by directly quantizing a configuration of multi-centered black holes. We begin by reviewing some relevant properties of these solutions. Consider a supersymmetric solution describing $n$ black holes, with different centers carrying charges $\alpha_{1}, \ldots, \alpha_{n} \in \tilde{\Gamma}$ located at $\vec{r}_{1}, \ldots, \vec{r}_{n}$. We shall define

$$
r_{i j}=\left|\vec{r}_{i}-\vec{r}_{j}\right|
$$

The equations governing the locations $\vec{r}_{i}$ are [7]

$$
\sum_{\substack{j=1 \\ j \neq i}}^{n} \frac{\alpha_{i j}}{r_{i j}}=c_{i},
$$

where

$$
c_{i} \equiv 2 \operatorname{Im}\left(e^{-\mathrm{i} \phi} Z_{\alpha_{i}}\right), \quad \phi=\arg \left(Z_{\alpha_{1}+\cdots+\alpha_{n}}\right) .
$$

Here $Z_{\alpha}$ denotes the central charge for the charge $\alpha$, computed with the asymptotic values of the moduli fields. The constants $c_{i}$ depend on the moduli through $Z_{\alpha}$, and satisfy $\sum_{i=1, \ldots, n} c_{i}=0 .^{9}$

First we shall show that in the chamber $c_{+}$none of these solutions exist. For this note that at the wall of marginal stability $\mathcal{P}\left(\gamma_{1}, \gamma_{2}\right)$ the central charges of $\gamma_{1}$ and $\gamma_{2}$, and hence of all the vectors $\alpha_{i}$, become aligned. As a result, near this wall the real parts of $e^{-\mathrm{i} \phi} Z_{\alpha_{i}}$ are all positive; we shall denote these by $A_{i}$. On the other hand it follows from (3.1) that for $i=1$ the left hand side of (3.18) is positive and for $i=n$ it is negative. Thus we must have

$$
e^{-i \phi} Z_{\alpha_{1}}=A_{1}+i B_{1}, \quad e^{-i \phi} Z_{\alpha_{n}}=A_{n}-i B_{n}, \quad A_{1}, B_{1}, A_{n}, B_{n}>0 .
$$

This gives a necessary condition for the multi-centered solution to exist,

$$
\left\langle\alpha_{1}, \alpha_{n}\right\rangle \operatorname{Im}\left(Z_{\alpha_{1}} \bar{Z}_{\alpha_{n}}\right)=\left\langle\alpha_{1}, \alpha_{n}\right\rangle\left(A_{1} B_{n}+A_{n} B_{1}\right)>0 .
$$

On the other hand the chamber $c_{+}$has been defined such that the right hand side of (3.21) is negative (see (2.6)). This shows that a multi-centered solution of the type analyzed above cannot exist in the chamber $c_{+}$. Note that this also proves that scaling solutions $[8,10,50]$, whose existence does not depend on the moduli, cannot exist for charges $\alpha_{1}, \ldots, \alpha_{n} \in \tilde{\Gamma}^{10}$

From now on we work in the chamber $c_{-}$. For an $n$-centered configuration, the location of the centers is specified by $3 n$ coordinates $\vec{r}_{i}(1 \leq i \leq n)$. Removing the trivial center

\footnotetext{
${ }^{9}$ In the original analysis of [7] each center was regarded as a single centered black hole. We shall use a slightly more general interpretation in which each center is allowed to be a black hole molecule - composite of two or more single centered black holes with charges of each center lying outside $\tilde{\Gamma}$. By working at a point close to the wall $\mathcal{P}\left(\gamma_{1}, \gamma_{2}\right)$ we can ensure that the distance between these molecules, denoted by $r_{i j}$ in eq.(3.18), is much larger than the internal size of each molecule, and hence (3.18) is a valid description of the inter-molecular distance for this configuration.

${ }^{10}$ We can also directly see this as follows. For the scaling solutions the right hand side of (3.18) vanishes [10]. Now for $i=1$ all the terms in the left hand side of (3.18) are manifestly positive due to the choice (3.1), making it impossible to satisfy this equation.
} 
of mass degrees of freedom we are left with $3 n-3$ coordinates. For multi-centered BPS solutions, the relative distances $r_{i j} \equiv\left|\vec{r}_{i}-\vec{r}_{j}\right|$ must satisfy (3.18) for $i=1, \ldots, n$. These equations are linearly dependent, since the sum over $i$ is trivially satisfied. This gives $(n-1)$ independent constraints. The moduli space of multi-centered solutions is then a $(2 n-2)$ dimensional space $\mathcal{M}_{n}\left(c_{1}, \ldots, c_{n}\right)$. In the case of interest here, where all $\alpha_{i}$ lie in $\tilde{\Gamma}, \mathcal{M}_{n}$ is compact.

As shown in [41], for fixed values of $c_{i}, \mathcal{M}_{n}$ carries a symplectic form $\omega$ given by the restriction of the two-form ${ }^{11}$

$$
\omega=\frac{1}{4} \sum_{i<j} \alpha_{i j} \epsilon^{a b c} \frac{\mathrm{d} r_{i j}^{a} \wedge \mathrm{d} r_{i j}^{b} r_{i j}^{c}}{\left|r_{i j}\right|^{3}},
$$

from $\mathbb{R}^{3 n-3}$ to the moduli space $\mathcal{M}_{n}\left(c_{1}, \ldots, c_{n}\right)$. This symplectic form is invariant under $\mathrm{SU}(2)$ rotations. The moment map associated to an infinitesimal rotation $\delta \vec{r}=\vec{\epsilon} \wedge \vec{r}$ is just $\vec{\epsilon} \cdot \vec{J}$, where

$$
\vec{J}=\frac{1}{2} \sum_{i<j} \alpha_{i j} \frac{\vec{r}_{i j}}{\left|r_{i j}\right|}
$$

is the angular momentum. Thus the spectrum of supersymmetric bound states can in principle be obtained from geometric quantization of this phase space and the information on angular momentum, required for computing $g_{\text {ref }}$, can be found by studying the $J_{3}$ eigenvalues of these bound states.

We now review the results of [41] for the bound state spectrum of 3-centered black holes in the chamber $c_{-}{ }^{12}$ In this case we have $\left\langle\alpha_{i}, \alpha_{j}\right\rangle \operatorname{Im}\left(Z_{\alpha_{i}} \bar{Z}_{\alpha_{j}}\right)>0$ and hence the clockwise ordering of the $\alpha_{i}$ 's will imply clockwise ordering of the $Z_{\alpha_{i}}$ 's. Furthermore we shall restrict the $\alpha_{i}$ 's to satisfy

$$
\alpha_{12}>\alpha_{23}
$$

so that the clockwise ordering of the $\alpha_{i}$ 's and their various linear combinations are given by eq.(3.8). Thus the same is true for the corresponding $Z$ 's. We now explicitly write out the equations (3.18) as follows:

$$
\frac{\alpha_{12}}{r_{12}}+\frac{\alpha_{13}}{r_{13}}=c_{1}, \quad-\frac{\alpha_{12}}{r_{12}}+\frac{\alpha_{23}}{r_{23}}=c_{2}, \quad \frac{\alpha_{13}}{r_{13}}+\frac{\alpha_{23}}{r_{23}}=-c_{3}, \quad c_{i} \equiv 2 \operatorname{Im}\left(e^{-\mathrm{i} \phi} Z_{\alpha_{i}}\right) .
$$

Since $e^{\mathrm{i} \phi}=Z_{\alpha_{1}+\alpha_{2}+\alpha_{3}} /\left|Z_{\alpha_{1}+\alpha_{2}+\alpha_{3}}\right|$, we have $c_{1}+c_{2}+c_{3}=0$. Furthermore since according to (3.8) $Z_{\alpha_{1}}$ and $Z_{\alpha_{1}+\alpha_{3}}$ precedes $Z_{\alpha_{1}+\alpha_{2}+\alpha_{3}}$ in the clockwise ordering while $Z_{\alpha_{2}}$ and $Z_{\alpha_{3}}$

\footnotetext{
${ }^{11}$ Our normalisation differs from a factor of two from the one used in [41]. This ensures that $\omega /(2 \pi)$ has integer periods.

${ }^{12}$ Note that if the three charge vectors $\alpha_{i}$ 's do not lie in a plane, then we can compute the bound state degeneracy using attractor flow trees [7], e.g. we could first approach a wall where the system splits into a pair of molecules, one with charge $\alpha_{1}+\alpha_{2}$ and another with charge $\alpha_{3}$ and so the index will be given by $(-1)^{\alpha_{13}+\alpha_{23}+1}\left(\alpha_{13}+\alpha_{23}\right) \Omega\left(\alpha_{1}+\alpha_{2}\right) \Omega\left(\alpha_{3}\right)$. Then we can approach another wall where the system with charge $\alpha_{1}+\alpha_{2}$ breaks up into a pair of molecules of charges $\alpha_{1}$ and $\alpha_{2}$ with index $(-1)^{\alpha_{12}+1} \alpha_{12} \Omega\left(\alpha_{1}\right) \Omega\left(\alpha_{2}\right)$. But when the three $\alpha_{i}$ 's are in the same plane spanned by $\gamma_{1}$ and $\gamma_{2}$, they all move away from each other at a similar rate when we approach the wall of marginal stability $\mathcal{P}\left(\gamma_{1}, \gamma_{2}\right)$ and we need to solve the 3-body bound state problem explicitly. Similarly if we have $n$ centers with their charges lying in a plane then we need to explicitly solve the $n$-body bound state problem.
} 
follow it, we have $c_{1} \geq 0, c_{1}+c_{3} \geq 0, c_{2} \leq 0, c_{3} \leq 0$. Thus we can parametrize the $c_{i}$ 's as

$$
c_{1}=a, \quad c_{2}=-a+b, \quad c_{3}=-b, \quad a, b \geq 0, \quad b \leq a .
$$

We can express the general solution to (3.25) as [41]

$$
r_{12}=\frac{\alpha_{12}}{\lambda-b}, \quad r_{23}=\frac{\alpha_{23}}{\lambda-a}, \quad r_{13}=\frac{\alpha_{13}}{a+b-\lambda},
$$

for some constant real parameter $\lambda$. The range of $\lambda$ is restricted by the positivity of each $r_{i j}$ and also the triangle inequality satisfied by the $r_{i j}$ 's. The positivity of the $r_{i j}$ 's together with (3.26) give $b \leq a \leq \lambda \leq a+b$. To study the consequences of the triangle inequality we express them as

$$
\begin{aligned}
& \frac{\alpha_{12}}{\lambda-b}+\frac{\alpha_{23}}{\lambda-a}-\frac{\alpha_{13}}{a+b-\lambda} \geq 0, \\
& \frac{\alpha_{23}}{\lambda-a}+\frac{\alpha_{13}}{a+b-\lambda}-\frac{\alpha_{12}}{\lambda-b} \geq 0, \\
& \frac{\alpha_{13}}{a+b-\lambda}+\frac{\alpha_{12}}{\lambda-b}-\frac{\alpha_{23}}{\lambda-a} \geq 0 .
\end{aligned}
$$

We need to find solutions to these inequalities in the range $a \leq \lambda \leq a+b$. We begin near $\lambda=a+\epsilon$ for some small $\epsilon$. At this point $r_{23}$ is large and the last of eqs.(3.28) is violated. As we increase $\lambda$, at some value the last equality is saturated when

$$
r_{23}=r_{13}+r_{12} .
$$

It is easy to see that at this point the other two inequalities hold and hence above this bound the allowed range of $\lambda$ begins. This continues till one of the other inequalities fail to hold. It is easy to see that second inequality continues to hold for $\lambda \leq a+b$ but the first inequality is violated beyond some value of $\lambda$ close to $a+b$ when

$$
r_{13}=r_{12}+r_{23} .
$$

The allowed range of angular momentum is given by the classical angular momentum carried by the system in the two extremes. At (3.29) the points $\left(\vec{r}_{2}, \vec{r}_{1}, \vec{r}_{3}\right)$ lie along a line and hence the angular momentum is given by $\left(\alpha_{13}+\alpha_{23}-\alpha_{12}\right) / 2$. On the other hand at $(3.30)$ the points $\left(\vec{r}_{1}, \vec{r}_{2}, \vec{r}_{3}\right)$ lie along a line and we have total angular momentum $\left(\alpha_{13}+\alpha_{23}+\alpha_{12}\right) / 2$. Thus we have

$$
J_{-}=\left(\alpha_{13}+\alpha_{23}-\alpha_{12}\right) / 2, \quad J_{+}=\left(\alpha_{13}+\alpha_{23}+\alpha_{12}\right) / 2 .
$$

As was shown in [41] in quantum theory the upper limit $J_{+}$is shifted to $J_{+}-1$ and states of all angular momentum between $J_{-}$and $J_{+}-1$ occur exactly once. This gives [41]

$$
\begin{aligned}
g_{\mathrm{ref}}\left(\alpha_{1}, \alpha_{2}, \alpha_{3} ; y\right) & =(-1)^{\alpha_{13}+\alpha_{23}+\alpha_{12}} \sum_{J_{+} J_{-}}^{J_{+}-1} \frac{y^{2 J+1}-y^{-2 J-1}}{y-y^{-1}} \\
& =(-1)^{\alpha_{13}+\alpha_{23}+\alpha_{12}} \frac{1}{\left(y-y^{-1}\right)^{2}}\left(y^{2 J_{+}}-y^{2 J_{-}}-y^{-2 J_{-}}+y^{-2 J_{+}}\right) \\
& =(-1)^{\alpha_{13}+\alpha_{23}+\alpha_{12}} \frac{1}{\sinh ^{2} \nu} \sinh \left(\nu\left(\alpha_{13}+\alpha_{23}\right)\right) \sinh \left(\nu \alpha_{12}\right),
\end{aligned}
$$

in agreement with (3.10). 
We shall now generalize eqs. (3.32) to an arbitrary number $n$ of centers. For this we shall first simplify (3.18). Since we are interested in the situation where the $\alpha_{i}$ 's lie in a two dimensional plane we have

$$
2 \operatorname{Im}\left(e^{-\mathrm{i} \phi} Z_{\alpha_{i}}\right)=\Lambda\left\langle\alpha_{i}, \sum_{j} \alpha_{j}\right\rangle=\Lambda \sum_{j \neq i} \alpha_{i j}
$$

for some positive constant $\Lambda$. This constant can be removed by a rescaling of the variables $\vec{r}_{i}$, but we shall keep it in our subsequent equations. This allows us to express (3.18) as:

$$
\sum_{\substack{j=1 \\ j \neq i}}^{n} \frac{\alpha_{i j}}{r_{i j}}=\Lambda \sum_{j \neq i} \alpha_{i j}
$$

Our strategy will be to relate $\operatorname{Tr}(-1)^{2 J_{3}} y^{2 J_{3}}$ to an integral over the classical phase space $\mathcal{M}_{n}$ of solutions to eq. (3.34). Now, $(-1)^{2 J_{3}}$ is a rapidly varying function on $\mathcal{M}_{n}$ and does not have a smooth classical limit. Our experience with the quantum theory for $n=2$ and $n=3$ nevertheless suggests that it takes the same value over all the quantum states and is given by $(-1)^{2 J_{\max }-n+1}$, where $J_{\max }=\sum_{i<j} \alpha_{i j} / 2$ is the maximum allowed classical angular momentum. On the other hand, for $y$ close to $1, y^{2 J_{3}}$ is a slowly varying function over the classical phase space and one expects that for large $\left|\alpha_{i j}\right|$, its quantum expectation value is well approximated by integrating $y^{2 J_{3}}$ over the classical phase space. Thus, we are led to consider

$$
g_{\text {classical }}\left(\left\{\alpha_{i}\right\}, y\right) \equiv \frac{(-1)^{\sum_{i<j} \alpha_{i j}-n+1}}{(2 \pi)^{n-1}(n-1) !} \int_{\mathcal{M}_{n}} e^{2 \nu J_{3}} \omega^{n-1}, \quad \nu \equiv \ln y .
$$

This formula should well approximate the refined index $g_{\text {ref }}$ at large $\left|\alpha_{i j}\right|$ and $y$ close to 1 , but could in principle be corrected in the full quantum theory. Our experience with the two and three centered cases, as well as an explicit evaluation of the 4-centered case presented below, suggests that at $y=1$ the classical phase space integral (3.35) in fact agrees with the exact quantum index $g\left(\left\{\alpha_{i}\right\}\right)$. In addition, the same integral (3.35), after a minor amendment to be described shortly, appears to agree with the exact refined index $g_{\text {ref }}\left(\left\{\alpha_{i}\right\}\right)$ for all values of $y$.

Now using the localization theorem of [51], we can express (3.35) as a sum over contributions from fixed points of the Hamiltonian vector field associated to the moment map $J_{3}$, i.e. rotations along the $z$-axis. Fixed points are therefore multi-centered black hole configurations in which all centers are aligned along the $z$-axis, in an appropriate order consistent with (3.34). Furthermore, since all the relative distances between the centers are fixed by (3.34), the fixed points are isolated. Thus, fixed points of $J_{3}$ are labelled by permutations $\sigma$ of $1,2, \ldots, n$ such that the centers are arranged in a given order along the $z$-axis, satisfying $z_{\sigma(i)}<z_{\sigma(j)}$ if $i<j$. In this case the constraint (3.34) takes the form

$$
\sum_{\substack{j=1 \\ j \neq i}}^{n} \frac{\alpha_{\sigma(i) \sigma(j)}}{z_{\sigma(j)}-z_{\sigma(i)}} \operatorname{sign}(j-i)=\Lambda \sum_{\substack{j=1 \\ j \neq i}}^{n} \alpha_{\sigma(i) \sigma(j)}
$$


which is equivalent to the extremization of the "superpotential"

$$
W=-\frac{1}{2} \sum_{i \neq j} \operatorname{sign}(j-i) \alpha_{\sigma(i) \sigma(j)} \log \left|z_{\sigma(j)}-z_{\sigma(i)}\right|-\Lambda \sum_{i \neq j} \alpha_{i j} z_{i}
$$

At such a fixed point the third component of the classical angular momentum is given by

$$
J_{3}=\frac{1}{2} \sum_{i<j} \alpha_{\sigma(i) \sigma(j)} .
$$

The localization formula of [51] now gives

$$
g_{\text {classical }}\left(\left\{\alpha_{i}\right\}, y\right)=(-1)^{\sum_{i<j} \alpha_{i j}+n-1}(2 \nu)^{1-n} \sum_{\sigma}^{\prime} s(\sigma) y^{\sum_{i<j} \alpha_{\sigma(i) \sigma(j)}},
$$

where $\sum_{\sigma}^{\prime}$ denotes sum over only those permutations for which a solution to (3.36) exists, and $s(\sigma)$ is the sign of the Hessian of the matrix representing the action of $2 \nu J_{3}$ on the tangent space of $\mathcal{M}_{n}$ at the fixed point. To compute $s(\sigma)$ we make a convenient choice of coordinates on $\mathcal{M}_{n}$. Without any loss of generality we can choose $\vec{r}_{1}$ to be at the origin. At a fixed point of the action of $J_{3}$, all the other points are then along the $z$-axis. We now note that to first order the relative distances between the centers remain unchanged if we displace each of the $\vec{r}_{i}$ for $i \geq 2$ in the $(x-y)$ plane. Thus these $(2 n-2)$ coordinates provide us with a convenient parametrization of the moduli space of the solution near this fixed point. Let us denote them by $\left(x_{i}, y_{i}\right)(2 \leq i \leq n)$. The action of $J_{3}$ on these coordinates is simply an independent rotation in the $\left(x_{i}, y_{i}\right)$ plane for each $i$. The Hessian of $2 \nu J_{3}$ is given by $(2 \nu)^{n-1}$ up to a sign $s(\sigma)$. To determine the sign we note that in the coordinate system $\left\{x_{i}, y_{i}\right\}$ introduced above, $J_{3}$ and $\omega$ take the form:

$$
J_{3}=\frac{1}{2} \sum_{i<j} \alpha_{\sigma(i) \sigma(j)}-\frac{1}{4} M_{i j}\left(x_{i} x_{j}+y_{i} y_{j}\right)+\cdots, \quad \omega=\frac{1}{2} M_{i j} d x_{i} \wedge d y_{j}+\cdots,
$$

where $\cdots$ denote higher order terms and

$$
M_{i i}=\alpha_{1 i} \frac{z_{i}}{\left|z_{i}\right|^{3}}+\sum_{k \neq i, k \geq 2} \alpha_{i k} \frac{z_{k}-z_{i}}{\left|z_{k}-z_{i}\right|^{3}}, \quad M_{i j}=-\alpha_{i j} \frac{z_{j}-z_{i}}{\left|z_{j}-z_{i}\right|^{3}} \quad \text { for } i \neq j, \quad i, j \geq 2 .
$$

It is worth noting that the matrix $M_{i j}$ is also equal to the Hessian of the superpotential (3.37) with respect to the $n-1$ variables $z_{2}, \ldots z_{n}$, with $z_{1}$ being set to zero. The sign $s(\sigma)$ of the Hessian associated with the fixed point is thus given by

$$
s(\sigma)=\operatorname{sign} \operatorname{det} M .
$$

Although the prescription (3.42) appears to treat $z_{1}$ on a different footing than the other $z_{i}$ due to the gauge condition $z_{1}=0$, one could just as well have computed $s(\sigma)$ using a symmetric gauge condition $\sum_{i=1 \ldots n} z_{i}=0$. Indeed, the same sign $s(\sigma)$ can be obtained as (the opposite of) the sign of the determinant of the Hessian of the superpotential

$$
\hat{W}=W+\frac{\lambda}{n} \sum_{i=1}^{n} z_{i}
$$


with respect to all $z_{i}, i=1 \ldots n$ and to the Lagrange multiplier $\lambda$. To see this, note that the Hessian of $\hat{W}$ with respect to $\left(\lambda, z_{1}, z_{i=2 \ldots n}\right)$ is given by

$$
\hat{M}=\left(\begin{array}{ccc}
0 & 1 / n & A \\
1 / n & \partial^{2} W / \partial z_{1}^{2} & W_{1} \\
A^{T} & W_{1}^{T} & M
\end{array}\right)
$$

where both $A$ and $W_{1}$ are $(n-1)$ dimensional row matrices, with $A=(1 / n, \cdots 1 / n)$ and $W_{1}=\left(\partial^{2} W / \partial z_{1} \partial z_{2}, \cdots \partial^{2} W / \partial z_{1} \partial z_{n}\right)$. By adding the third to $(n+1)$ 'th rows to the second row and third to $(n+1)$-th columns to the second column and using the fact that $\sum_{i=1}^{n} \partial^{2} W / \partial z_{i} \partial z_{j}=0$ due to translation invariance, we can bring (3.44) to the form

$$
\left(\begin{array}{ccc}
0 & 1 & A \\
1 & 0 & \mathbf{0} \\
A^{T} & \mathbf{0}^{T} & M
\end{array}\right)
$$

where $\mathbf{0}$ denotes an $(n-1)$-dimensional row matrix with all entries 0 . From this we see that $\operatorname{det} \hat{M}=-\operatorname{det} M$.

If there are more than one solution of (3.36) for a given permutation $\sigma$ then the right hand side of (3.42) should be replaced by a sum of $\operatorname{sign} \operatorname{det} M$ over all solutions. Numerical evidence indicates however that there is at most one fixed point for a given permutation. Moreover, it suggests that $s(\sigma)$ can be expressed in terms of the permutation $\sigma$ through

$$
s(\sigma)=(-1)^{\#\{i ; \sigma(i+1)<\sigma(i)\}} .
$$

This is easily proven for the special critical points of $W$ with $\operatorname{sign}\left(z_{\sigma(i)}-z_{\sigma(j)}\right)=\operatorname{sign}(i-j)$ and $\operatorname{sign}\left(z_{\sigma(i)}-z_{\sigma(j)}\right)=\operatorname{sign}(j-i)$. These represent solutions of (3.36) corresponding to permutations $(12 \ldots n)$ and $(n \ldots 21)$. Since these correspond to the global maximum and global minimum of $J_{3}$, respectively, the matrix $M_{i j}$ is positive definite or negative definite, respectively, leading to $s(12 \ldots n)=1, s(n \ldots 21)=(-1)^{n-1}$. As we shall see in $\S 3.3$, the result (3.46) is required for consistency with the Higgs branch derivation presented in $\S 3.1$. This also suggests that if there are more than one fixed points for a given permutation their contributions should cancel pairwise leaving behind the contribution from 0 or 1 fixed point.

We do not expect the classical formula (3.35) and hence (3.39) to reproduce the full $y$ dependent quantum answer for $\operatorname{Tr}(-y)^{2 J_{3}}$ — after all the quantization of angular momentum is not visible classically. This is apparent from (3.39): while the terms inside the sum involve integral powers of $y=e^{\nu}$ and hence are compatible with charge quantization, the overall factor $(2 \nu)^{1-n}$ does not respect charge quantization. Comparison with the exact results $(3.2),(3.32)$ suggests a remedy: ${ }^{13}$ replace the factor of $(2 \nu)^{1-n}$ by $(2 \sinh \nu)^{1-n}=\left(y-y^{-1}\right)^{1-n}$. In the $y \rightarrow 1$ limit this will approach the classical result in accordance with the earlier observation that in this limit the classical and quantum results agree. Thus our proposal for the full quantum version of (3.39) is

$$
g_{\mathrm{ref}}\left(\left\{\alpha_{i}\right\}, y\right)=(-1)^{\sum_{i<j} \alpha_{i j}+n-1}\left(y-y^{-1}\right)^{1-n} \sum_{\sigma}^{\prime} s(\sigma) y^{\sum_{i<j} \alpha_{\sigma(i) \sigma(j)}}
$$

\footnotetext{
${ }^{13}$ See note added in proof.
} 
This reduces the problem of computing the function $g_{\text {ref }}$ to identifying which of the permutations $\sigma$ are consistent with (3.36). This is a tedious but straightforward procedure. Below we give the results for $n=3$ and $n=4$ for the same order of various linear combinations of the $\alpha_{i}$ 's as given in (3.8) and (3.13).

For $n=3$ the detailed analysis of the configuration space was carried out in [41] some relevant details of which were reviewed earlier in this section. Two of the four collinear configurations are given in (3.29) and two others are given by (3.30). ${ }^{14}$ This gives the following order of the centers along the $z$-axis and the value of $s(\sigma)$,

$$
\{1,2,3 ;+\},\{2,1,3 ;-\},\{3,1,2 ;-\},\{3,2,1 ;+\}
$$

leading to

$$
\begin{aligned}
g_{\mathrm{ref}}\left(\alpha_{1}, \alpha_{2}, \alpha_{3}, y\right)= & (-1)^{\alpha_{12}+\alpha_{23}+\alpha_{13}}\left(y-y^{-1}\right)^{-2} \\
& \times\left(y^{\alpha_{12}+\alpha_{13}+\alpha_{23}}-y^{\alpha_{13}+\alpha_{23}-\alpha_{12}}-y^{\alpha_{12}-\alpha_{23}-\alpha_{13}}+y^{-\alpha_{12}-\alpha_{13}-\alpha_{23}}\right),
\end{aligned}
$$

in agreement with the result (3.32) following from exact quantization of the 3-centered system.

Next we test (3.47) by working out the result for $n=4$. There are 12 fixed points, whose orders and the associated $s(\sigma)$ are given by

$$
\begin{aligned}
& \{1,2,3,4 ;+\},\{3,1,2,4 ;-\},\{1,3,4,2 ;-\},\{1,4,2,3 ;-\},\{3,1,4,2 ;+\},\{2,3,4,1 ;-\}, \\
& \{1,4,3,2 ;+\},\{2,4,1,3 ;-\},\{3,2,4,1 ;+\},\{2,4,3,1 ;+\},\{4,2,1,3 ;+\},\{4,3,2,1 ;-\} .
\end{aligned}
$$

eq.(3.47) now gives

$$
\begin{aligned}
& g_{\mathrm{ref}}\left(\alpha_{1}, \alpha_{2}, \alpha_{3}, \alpha_{4}, y\right)=(-1)^{\alpha_{12}+\alpha_{13}+\alpha_{14}+\alpha_{23}+\alpha_{24}+\alpha_{34}+1}\left(y-y^{-1}\right)^{-3} \\
& \times\left(y^{\alpha_{12}+\alpha_{13}+\alpha_{14}+\alpha_{23}+\alpha_{24}+\alpha_{34}}-y^{\alpha_{12}-\alpha_{13}+\alpha_{14}-\alpha_{23}+\alpha_{24}+\alpha_{34}}-y^{\alpha_{12}+\alpha_{13}+\alpha_{14}-\alpha_{23}-\alpha_{24}+\alpha_{34}}\right. \\
& \quad-y^{\alpha_{12}+\alpha_{13}+\alpha_{14}+\alpha_{23}-\alpha_{24}-\alpha_{34}}+y^{\alpha_{12}-\alpha_{13}+\alpha_{14}-\alpha_{23}-\alpha_{24}+\alpha_{34}}-y^{-\alpha_{12}-\alpha_{13}-\alpha_{14}+\alpha_{23}+\alpha_{24}+\alpha_{34}} \\
& \quad+y^{\alpha_{12}+\alpha_{13}+\alpha_{14}-\alpha_{23}-\alpha_{24}-\alpha_{34}}-y^{-\alpha_{12}+\alpha_{13}-\alpha_{14}+\alpha_{23}+\alpha_{24}-\alpha_{34}}+y^{-\alpha_{12}-\alpha_{13}-\alpha_{14}-\alpha_{23}+\alpha_{24}+\alpha_{34}} \\
& \left.\quad+y^{-\alpha_{12}-\alpha_{13}-\alpha_{14}+\alpha_{23}+\alpha_{24}-\alpha_{34}}+y^{-\alpha_{12}+\alpha_{13}-\alpha_{14}+\alpha_{23}-\alpha_{24}-\alpha_{34}}-y^{-\alpha_{12}-\alpha_{13}-\alpha_{14}-\alpha_{23}-\alpha_{24}-\alpha_{34}}\right)
\end{aligned}
$$

in agreement with (3.14).

\subsection{Comparison of the results of Higgs branch and Coulomb branch analysis}

To compare the results of the Coulomb branch analysis described in $\S 3.2$ with the Higgs branch computation described in $\S 3.1$, note that the power $y^{\sum_{i<j} \alpha_{\sigma(i) \sigma(j)}}$ in (3.47) matches the power of $y$ in (3.2), provided the ordered decomposition $\beta$ is chosen as follows: break

\footnotetext{
${ }^{14}$ For each of (3.29) and (3.30) we have two configurations related by $z \rightarrow-z$ symmetry.
} 
the sequence $\{\sigma(i), i=1, \ldots, n\}$ into increasing subsequences $\left\{\sigma\left(i_{a-1}+1\right), \ldots, \sigma\left(i_{a}\right)\right\}$, $0=i_{0}<i_{1}<\cdots<i_{s}=n$, where $s$ is the number of such increasing subsequences. The vectors $\beta^{(a)}$ are then

$$
\beta^{(s+1-a)}=\sum_{i=i_{a-1}+1}^{i=i_{a}} \alpha_{\sigma(i)} .
$$

For example for the permutation $\sigma(123)=(132)$ there are two possible choices for the increasing subsequences: $\{\{13\},\{2\}\}$ and $\{\{1\},\{3\},\{2\}\}$, and the corresponding partitions are $\left\{\alpha_{2}, \alpha_{1}+\alpha_{3}\right\}$ and $\left\{\alpha_{2}, \alpha_{3}, \alpha_{1}\right\}$. To see that the partition (3.52) generates the correct power of $y$ associated with the permutation $\sigma$, we note that (3.52) implies that $m_{i}^{s+1-a}=$ $\sum_{j=i_{a-1}+1}^{i_{a}} \delta_{i, \sigma(j)}$ in (3.2). Leaving aside the factor of $\left(y-y^{-1}\right)^{-n+1}$, the power of $y$ in (3.2) is now given by

$$
-\sum_{i<j} \alpha_{i j}+2 \sum_{a \geq b} \sum_{k=i_{a-1}+1}^{i_{a}} \sum_{\substack{l=i_{b-1}+1 \\ \sigma(l)<\sigma(k)}}^{i_{b}} \alpha_{\sigma(l) \sigma(k)}=-\sum_{i<j} \alpha_{i j}+2 \sum_{\substack{l<k \\ \sigma(l)<\sigma(k)}} \alpha_{\sigma(l) \sigma(k)},
$$

where in the second step we have used the fact that if $k, l$ belong to different subsequences labeled by $a$ and $b$ then $a>b$ will imply $k>l$. On the other hand if they belong to the same subsequence then, since the subsequence is increasing, the condition $\sigma(l)<\sigma(k)$ will imply $l<k$. The right hand side of (3.53) gives precisely the power of $y$ associated with the permutation $\sigma$ as given in (3.47).

The sign associated with the partition $(3.52)$ in $(3.2)$ is $(-1)^{s-1}$. If the increasing subsequences are maximal, i.e. chosen so that it is not possible to build bigger increasing subsequences, then this sign is in agreement with the rule given in (3.46). For example, for the permutation $\sigma(1234)=3142$ the maximal increasing subsequences are $\{\{3\},\{14\},\{2\}\}$. Thus it corresponds to the ordered decomposition $\left\{\alpha_{2}, \alpha_{1}+\alpha_{4}, \alpha_{3}\right\}$, contributing with a positive sign.

Now, there are typically many increasing subsequences associated with a given permutation, obtained by breaking up the maximal increasing subsequences into smaller increasing subsequences, and contributing with different signs. Thus, in order to determine if a given permutation contributes and with what sign, we have to combine the contributions from these different terms. In the previous example the other possible increasing subsequence is $\{\{3\},\{1\},\{4\},\{2\}\}$, but the corresponding partition $\left\{\alpha_{2}, \alpha_{4}, \alpha_{1}, \alpha_{3}\right\}$ does not satisfy the condition (3.3) and hence does not contribute. In general however the situation is more complicated. Consider for example the permutation $\sigma(1234)=(3412)$. The corresponding increasing subsequences are $\{\{34\},\{12\}\},\{\{3\},\{4\},\{12\}\},\{\{34\},\{1\},\{2\}\}$, and $\{\{3\},\{4\},\{1\},\{2\}\}$, associated to the partitions $\left\{\alpha_{1}+\alpha_{2}, \alpha_{3}+\alpha_{4}\right\},\left\{\alpha_{1}+\alpha_{2}, \alpha_{4}, \alpha_{3}\right\}$, $\left\{\alpha_{2}, \alpha_{1}, \alpha_{3}+\alpha_{4}\right\}$ and $\left\{\alpha_{2}, \alpha_{1}, \alpha_{4}, \alpha_{3}\right\}$, respectively. For the order given in (3.13) only the first and the third partitions are allowed by the rules (3.3). They contribute with opposite sign making the contribution vanish. This explains why the permutation (3412) is absent from the list (3.50).

It is easy to convince oneself that all possible partitions of the vectors $\left(\alpha_{1}, \ldots, \alpha_{n}\right)$, whether or not they satisfy the condition (3.3), are in one to one correspondence with the 
set of all increasing subsequences of all the permutations of $(12 \ldots n)$ via the rule $(3.52)$. So, the complete Higgs branch contribution can be generated by beginning with the maximal increasing subsequences associated with a given permutation and combining them with the contribution from other increasing subsequences associated with the same permutation. The following observations are now in order:

1. If a given partition is not allowed by the rule (3.3) then all its subpartitions are also disallowed. For example in the example of the previous paragraph, once we know that $\left\{\alpha_{1}+\alpha_{2}, \alpha_{4}, \alpha_{3}\right\}$ is not allowed, we can immediately conclude that $\left\{\alpha_{2}, \alpha_{1}, \alpha_{4}, \alpha_{3}\right\}$ is also not allowed.

2. If there are two or more maximal increasing subsequences of length two or more, then each of the maximal increasing subsequences can be independently broken up into smaller increasing subsequences. The compatibility of a partition of a particular maximal increasing sequence with the condition (3.3) or not is independent on the partitioning of the other maximal increasing subsequences. For example for the partition $\left\{\alpha_{1}+\alpha_{2}, \alpha_{3}+\alpha_{4}\right\}$, the compatibility of the splitting of $\alpha_{3}+\alpha_{4}$ into $\left\{\alpha_{4}, \alpha_{3}\right\}$ can be determined independently of whether $\alpha_{1}+\alpha_{2}$ is kept as a single element, or has been split into $\left\{\alpha_{2}, \alpha_{1}\right\}$. For this reason we can associate, to each maximal increasing subsequence, a weight given by a sum of \pm 1 for each of the allowed splittings of that subsequence $(+1$ for splitting into odd number of subsequences, including the original maximal increasing subsequence, and -1 for splitting into even number of subsequences). The final weight is given by the product of the weights computed from each maximal increasing subsequence.

In the example above the weight factor associated with $\alpha_{3}+\alpha_{4}$ is 1 since it cannot be split, while the weight factor associated with $\alpha_{1}+\alpha_{2}$ vanishes since it allows a split $\left\{\alpha_{2}, \alpha_{1}\right\}$ with opposite sign. As a result the net weight is $1 \times 0=0$.

3. The problem of determining the contribution from a given permutation now reduces to computing the weight factor associated with each maximal increasing subsequence of that permutation. This can be done as follows. We begin with a particular maximal increasing subsequence and first consider all possible partitions of this subsequence into two smaller increasing subsequences. For this we need to simply insert a comma at one place that indicates how we divide the original subsequence. Not all such subsequences may generate partitions allowed by (3.3); let us assume that there are $k$ possible places where we are allowed to insert the comma. This gives $k$ terms, each with weight -1 . Now consider the possible partitions of the same maximal increasing subsequence into three increasing subsequences. It follows from the rule (3.3) that the allowed partitions are obtained by inserting a pair of commas into two of the same $k$ possible positions. Thus there are $k(k-1) / 2$ possible terms, each with weight 1. This generalizes to partitioning into arbitrary number of increasing subsequences. Thus the net weight factor is $1-k+\left(\begin{array}{l}k \\ 2\end{array}\right)-\left(\begin{array}{l}k \\ 3\end{array}\right)+\cdots=(1-1)^{k}$. This shows that the weight factor vanishes for $k \geq 1$, and is 1 for $k=0$ i.e. when it is not possible to subpartition an increasing subsequence satisfying (3.3). 
This leads to the following simple rule for deciding when a given permutation contributes and the sign of the contribution: A given permutation contributes if its maximal increasing subsequences generate a partition satisfying (3.3) via (3.52), and none of the other (non-maximal) increasing subsequences generate an allowed partition. The sign of the contribution is given by (3.46).

We should of course keep in mind that the algorithm described above is not an independent result derived from the Coulomb branch, it is required to ensure that the Coulomb and the Higgs branch results agree. It will be interesting to find an independent derivation of this from the Coulomb branch analysis by directly examining the condition for the existence of solutions to (3.34).

Before concluding this section we shall demonstrate how the algorithm given above can be used to give a derivation of the semi-primitive wall-crossing formula. We choose $\alpha_{n}$ to be $\gamma_{1}$ and $\alpha_{1}, \cdots \alpha_{n-1}$ to consist of $m_{1}$ copies of $\gamma_{2}, m_{2}$ copies of $2 \gamma_{2}$ etc. in some fixed order (which can be decided by adding some arbitrary small vector to each of these charges which will be taken to zero at the end). Thus we have $n=1+\sum_{s} m_{s}$ and $\sum_{i<j} \alpha_{i j}=-\gamma_{12} \sum_{s} s m_{s}$. Since $\left\langle\gamma_{1}+k \gamma_{2}, \gamma_{1}+\sum_{s} s m_{s} \gamma_{2}\right\rangle<0$ for $k<\sum_{s} s m_{s}$, it follows from the (3.3) that $\gamma_{1}$ must be part of the last partition. Consider now a permutation of $1, \cdots n$. In order that $\gamma_{1}$ is part of the last partition, the first maximal increasing subsequence in this permutation must contain the element $n$ as its last element. This subsequence cannot be partitioned into smaller increasing subsequences since then $\gamma_{1}$ will not be part of the last partition. Furthermore in order that the permutation gives a nonvanishing contribution the rest of the maximal increasing subsequences must each have length 1 , since any maximal increasing subsequence of length 2 or more can be partitioned into smaller increasing subsequences without violating (3.3) and the result will vanish. This implies that the rest of the elements must be arranged in decreasing order in the permutation $\sigma$. Thus the only freedom in choosing the permutation is in deciding which elements are part of the first increasing subsequence. Let there be $k_{s}$ copies of $s \gamma_{2}$ in this set; this can be chosen in a total of $\prod_{s}\left(\begin{array}{c}m_{s} \\ k_{s}\end{array}\right)$ ways. The total number of partitions associated with this permutation is $1+\sum_{s}\left(m_{s}-k_{s}\right)$. Thus the net contribution to $g_{\text {ref }}$ is given by

$$
\begin{aligned}
(-y)^{\sum_{s} m_{s}+\gamma_{12} \sum_{s} m_{s} s}\left(y^{2}-1\right)^{-\sum_{s} m_{s}} \prod_{s} \sum_{k_{s}=0}^{m_{s}}\left(\begin{array}{c}
m_{s} \\
k_{s}
\end{array}\right)(-1)^{m_{s}-k_{s}} y^{-2 \gamma_{12} k_{s} s} & \\
& =\prod_{s}\left[\frac{(-y)^{\left\langle\gamma_{1}, s \gamma_{2}\right\rangle}-(-y)^{-\left\langle\gamma_{1}, s \gamma_{2}\right\rangle}}{y-y^{-1}}\right]^{m_{s}}
\end{aligned}
$$

in agreement with (2.42).

\section{Wall crossing from the Kontsevich-Soibelman formula}

The first solution to the problem of determining $g\left(\left\{\alpha_{i}\right\}\right)$ was given by Kontsevich and Soibelman [11] and also independently by Joyce and Song [14, 15]. In this section we shall review the results of [11] and compare them with our results. Subsection 4.1 states the 
KS wall-crossing formula, subsection 4.2 explains charge conservation and the following subsections apply the KS formula to determine the jump of $\Omega(\gamma)$ in various cases. In subsections 4.7 and 4.8 we describe generalization of the KS formula to the motivic index.

\subsection{The KS formula}

To state the KS formula, we introduce the Lie algebra $\mathcal{A}$ spanned by abstract generators $\left\{e_{\gamma}, \gamma \in \Gamma\right\}$, satisfying the commutation rule

$$
\left[e_{\gamma}, e_{\gamma^{\prime}}\right]=\kappa\left(\left\langle\gamma, \gamma^{\prime}\right\rangle\right) e_{\gamma+\gamma^{\prime}}
$$

where we defined

$$
\kappa(x)=(-1)^{x} x .
$$

At a given point in moduli space labeled by the parameters $\left\{t^{a}\right\}$, we introduce the operator

$$
U_{\gamma}\left(t^{a}\right)=\exp \left(\Omega\left(\gamma ; t^{a}\right) \sum_{d=1}^{\infty} \frac{e_{d \gamma}}{d^{2}}\right)
$$

in the Lie group generated by $\mathcal{A}$. The KS wall-crossing formula $[11,17]$ states that the product

$$
A_{\gamma_{1}, \gamma_{2}}=\prod_{\substack{\gamma=M \gamma_{1}+N \gamma_{2} \\ M \geq 0, N \geq 0}} U_{\gamma}
$$

ordered so that as we move from the left to the right the corresponding $Z_{\gamma}$ 's are ordered clockwise, i.e. according to decreasing values of $\arg \left(Z_{\gamma}\right)$, stays constant across the hyperplane of marginal stability $\mathcal{P}\left(\gamma_{1}, \gamma_{2}\right)$. As $t^{a}$ crosses this locus, $\Omega\left(\gamma ; t^{a}\right)$ jumps and the order of the factors is reversed, but the operator $A_{\gamma_{1}, \gamma_{2}}$ stays constant. Thus, the KS formula may be stated as the equality

$$
\prod_{\substack{M \geq 0, N \geq 0, M / N \downarrow}} U_{M \gamma_{1}+N \gamma_{2}}^{+}=\prod_{\substack{M \geq 0, N \geq 0, M / N \uparrow}} U_{M \gamma_{1}+N \gamma_{2}}^{-}
$$

where $M / N \downarrow$ means that the terms in the product are arranged from left to right in the order of decreasing values of $M / N$ while $M / N \uparrow$ implies opposite ordering of the factors, and $U_{\gamma}^{ \pm}$are defined as in (4.3) with $\Omega\left(\gamma ; t^{a}\right)$ replaced by $\Omega^{ \pm}$.

Noting that the operators $U_{k \gamma}$ for different $k \geq 1$ commute, one may combine them into

$$
V_{\gamma} \equiv \prod_{k=1}^{\infty} U_{k \gamma}=\exp \left(\sum_{\ell=1}^{\infty} \bar{\Omega}(\ell \gamma) e_{\ell \gamma}\right), \quad \bar{\Omega}(\gamma)=\sum_{m \mid \gamma} m^{-2} \Omega(\gamma / m)
$$

and rewrite (4.4) into a product over primitive vectors only,

$$
A_{\gamma_{1}, \gamma_{2}}=\prod_{\substack{\gamma=M \gamma_{1}+N \gamma_{2}, M \geq 0, N \geq 0, \operatorname{gcd}(M, N)=1}} V_{\gamma}
$$


Using the definition of $\Omega^{ \pm}$given in $\S 2.2$ the wall-crossing formula takes the form

$$
\prod_{\substack{M \geq 0, N \geq 0, \operatorname{gcd}(M, N)=1, M / N \downarrow}} V_{M \gamma_{1}+N \gamma_{2}}^{+}=\prod_{\substack{M \geq 0, N \geq 0, \operatorname{gcd}(M, N)=1, M / N \uparrow}} V_{M \gamma_{1}+N \gamma_{2}}^{-},
$$

where $V_{\gamma}^{ \pm}$are defined as in (4.6) with $\bar{\Omega}$ replaced by $\bar{\Omega}^{ \pm}$.

The invariants $\bar{\Omega}^{-}\left(M \gamma_{1}+N \gamma_{2}\right)$ on one side of the wall can be determined in terms of the invariants $\bar{\Omega}^{+}\left(M \gamma_{1}+N \gamma_{2}\right)$ on the other side by expressing both sides of (4.8) into single exponentials using the Baker-Cambell-Hausdorff (BCH) formula, and then comparing the coefficients of each $e_{\gamma}$ on either side. These equations can be solved iteratively to determine $\bar{\Omega}^{-}\left(M \gamma_{1}+N \gamma_{2}\right)$ in terms of the $\bar{\Omega}^{+}$'s, starting with the lowest values of $(M, N)$. This is most conveniently done by projecting the relation (4.8) to the finite-dimensional algebra

$$
\mathcal{A}_{M, N}=\mathcal{A} /\left\{\sum_{m>M \text { and } / \text { or } n>N} \mathbb{R} \cdot e_{m \gamma_{1}+n \gamma_{2}}\right\},
$$

and using the Baker-Campbell-Hausdorff (BCH) formula to commute the factors (see later). Since $\mathcal{A}_{M, N}$ is a finite dimensional algebra generated by $m \gamma_{1}+n \gamma_{2}$ for $0 \leq m \leq M$ and $0 \leq n \leq N$, we have a finite number of equations relating $\Omega^{-}\left(m \gamma_{1}+n \gamma_{2}\right)$ to $\Omega^{+}\left(m \gamma_{1}+n \gamma_{2}\right)$. For example a trivial consequence of (4.8) is the relation

$$
\Omega^{-}\left(M \gamma_{1}+N \gamma_{2}\right)=\Omega^{+}\left(M \gamma_{1}+N \gamma_{2}\right)
$$

whenever $M=0$ or $N=0$. This follows from the fact the algebras $\mathcal{A}_{M, 0}$ and $\mathcal{A}_{0, N}$ are commutative.

In order to derive the semi-primitive wall-crossing formula and generalizations thereof, it is also practical to work with the infinite dimensional algebra $\mathcal{A}_{M, \infty}$, and consider the generating functions

$$
Z^{ \pm}(M, q)=\sum_{N=0}^{\infty} \Omega^{ \pm}\left(M \gamma_{1}+N \gamma_{2}\right) q^{N}, \quad \bar{Z}^{ \pm}(M, q)=\sum_{N=0}^{\infty} \bar{\Omega}^{ \pm}\left(M \gamma_{1}+N \gamma_{2}\right) q^{N}
$$

for fixed value of $M$. These two objects are related by

$$
\bar{Z}^{ \pm}(M, q)=\sum_{d \mid M} \frac{1}{d^{2}} Z^{ \pm}\left(M / d, q^{d}\right), \quad Z^{ \pm}(M, q)=\sum_{d \mid M} \frac{\mu(d)}{d^{2}} \bar{Z}^{ \pm}\left(M / d, q^{d}\right)
$$

\subsection{Charge conservation from KS formula}

In this section we shall draw attention to one specific feature of the wall-crossing formula given in (4.8), namely 'charge conservation'. It follows from the algebra (4.1), and the definition of $V_{\gamma}$ given in (4.6) that after combining each side into a single exponential, the coefficient of $e_{\gamma}$ consists of a sum of products of the form $\prod_{i} \bar{\Omega}\left(\gamma_{i}\right)$ with $\sum_{i} \gamma_{i}=\gamma$, up to an overall numerical constant. Thus, any relation that follows from (4.8) has the property that the sum of the charges in the argument of $\bar{\Omega}$ 's has the same value for all the terms on either side of (4.8). Thus when we solve this to find $\bar{\Omega}^{-}(\gamma)$ in terms of 
$\bar{\Omega}^{+}(\gamma)$ and products of $\bar{\Omega}^{+}\left(\gamma_{i}\right)$ 's, each term in the expression will have the property that the charges in the argument of $\bar{\Omega}^{+}$in the product will add up to $\gamma$. This is precisely the 'charge conservation' rule that followed from the use of Maxwell-Boltzmann statistics in $\S 2.4$. Note that the wall-crossing formula written in terms of $\Omega^{ \pm}$does not have any such manifest charge conservation. We shall see examples of this 'charge conservation' rule in the explicit examples to be described below.

When several walls are crossed consecutively, the black hole molecules and bound molecular clusters can be decomposed into smaller molecules and eventually just single atoms. This is the attractor flow conjecure [7]. Knowing the indices of the atoms, one can in principle determine the contribution to the index of the total molecule, or equivalently flow tree. These flow trees are naturally parametrized by nested lists, e.g. $\left(\left(\gamma_{1}, \gamma_{2}\right), \gamma_{3}\right)$, which need to satisfy the 'charge conservation' rule. The structure of the nested lists is identical to the commutation relations of the KS formula in terms of $\bar{\Omega}(\gamma)$, and allows to determine easily the contribution of a molecule to the index [29].

\subsection{Primitive wall-crossing}

In $\mathcal{A}_{1,1}$ the $\mathrm{BCH}$ formula reduces to

$$
e^{X} e^{Y}=e^{X+Y+\frac{1}{2}[X, Y]}
$$

since all multiple commutators involving three or more generators vanish. The wall-crossing equation takes the form

$$
\begin{aligned}
& \exp \left(\bar{\Omega}^{+}\left(\gamma_{1}\right) e_{\gamma_{1}}\right) \exp \left(\bar{\Omega}^{+}\left(\gamma_{1}+\gamma_{2}\right) e_{\gamma_{1}+\gamma_{2}}\right) \exp \left(\bar{\Omega}^{+}\left(\gamma_{2}\right) e_{\gamma_{2}}\right) \\
&=\exp \left(\bar{\Omega}^{-}\left(\gamma_{2}\right) e_{\gamma_{2}}\right) \exp \left(\bar{\Omega}^{-}\left(\gamma_{1}+\gamma_{2}\right) e_{\gamma_{1}+\gamma_{2}}\right) \exp \left(\bar{\Omega}^{-}\left(\gamma_{1}\right) e_{\gamma_{1}}\right) .
\end{aligned}
$$

From this we find the primitive wall-crossing relation

$$
\bar{\Omega}^{-}(1,1)=\bar{\Omega}^{+}(1,1)+(-1)^{\gamma_{12}} \gamma_{12} \bar{\Omega}^{+}(0,1) \bar{\Omega}^{+}(1,0),
$$

where for simplicity we have denoted $\bar{\Omega}^{ \pm}\left(M \gamma_{1}+N \gamma_{2}\right)$ by $\bar{\Omega}^{ \pm}(M, N)$ and $\left\langle\gamma_{1}, \gamma_{2}\right\rangle$ by $\gamma_{12}$.

\subsection{Generic 3-body and 4-body contributions}

We shall now extract the generic $n$-body contribution from the KS wall-crossing formula. To explain what is meant by 'generic', let $n=3$, and $\alpha_{1}, \alpha_{2}, \alpha_{3}$ be three distinct (not necessarily primitive) elements of $\tilde{\Gamma}$ such that their central charges $Z_{\alpha_{i}}, i=1,2,3$ are arranged in clockwise order in $c_{-}$. Then $\alpha_{i j} \equiv\left\langle\alpha_{i}, \alpha_{j}\right\rangle>0$ for $i<j$. For definiteness we shall choose the $\alpha_{i}$ 's such that $\alpha_{12}>\alpha_{23}$. In this case, in the convention described below (3.1), the different linear combinations of the $\alpha_{i}$ 's will follow clockwise order as we move from left to right in the list (3.8). Furthermore, as we move from the left to the right in this list, the central charges will follow clockwise order in the chamber $c^{-}$and anticlockwise order in the chamber $c^{+}$. We can now ask the following question: what is the coefficient of $\bar{\Omega}^{+}\left(\alpha_{1}\right) \bar{\Omega}^{+}\left(\alpha_{2}\right) \bar{\Omega}^{+}\left(\alpha_{3}\right)$ in the expression of $\bar{\Omega}^{-}\left(\alpha_{1}+\alpha_{2}+\alpha_{3}\right)-\bar{\Omega}^{+}\left(\alpha_{1}+\alpha_{2}+\alpha_{3}\right)$ in terms of sum of products of $\bar{\Omega}^{+}$'s ? We refer to this coefficient as the generic 3-body 
contribution to wall-crossing. In order that the KS formula be consistent with the explicit computation of bound state degeneracies of black hole molecules, this coefficient must agree with the quantity $g\left(\alpha_{1}, \alpha_{2}, \alpha_{3}\right)$ computed in $\S 3$.

In order to carry out this computation, we can pretend that all $\bar{\Omega}^{+}(\alpha)$ other than those for $\alpha=\alpha_{1}, \alpha_{2}, \alpha_{3}$ vanish. In this case the left hand side of (4.8) takes a simple form $V_{\alpha_{3}}^{+} V_{\alpha_{2}}^{+} V_{\alpha_{1}}^{+}$. Our task is to manipulate this product so that in the final expression $V_{\alpha}$ 's follow the ordering of the $\alpha$ 's given in (3.8). For this it is most expedient to expand each $V_{\alpha}^{ \pm}$in (4.8) in a Taylor series expansion in $\bar{\Omega}^{ \pm}(\alpha)$, manipulate the left hand side so that in each term the $e_{\alpha}$ 's follow the order (3.8), and then identify the coefficients of each term on two sides. Since our goal is to find the term involving $\bar{\Omega}^{+}\left(\alpha_{1}\right) \bar{\Omega}^{+}\left(\alpha_{2}\right) \bar{\Omega}^{+}\left(\alpha_{3}\right)$, we can focus on the term $\bar{\Omega}^{+}\left(\alpha_{1}\right) \bar{\Omega}^{+}\left(\alpha_{2}\right) \bar{\Omega}^{+}\left(\alpha_{3}\right) e_{\alpha_{3}} e_{\alpha_{2}} e_{\alpha_{1}}$ in the Taylor series expansion on the left hand side. Repeated use of (4.1) gives

$$
\begin{aligned}
e_{\alpha_{3}} e_{\alpha_{2}} e_{\alpha_{1}}= & e_{\alpha_{1}} e_{\alpha_{2}} e_{\alpha_{3}}+\kappa\left(\left\langle\alpha_{2}, \alpha_{1}\right\rangle\right) e_{\alpha_{1}+\alpha_{2}} e_{\alpha_{3}}+\kappa\left(\left\langle\alpha_{3}, \alpha_{1}\right\rangle\right) e_{\alpha_{1}+\alpha_{3}} e_{\alpha_{2}} \\
& +\kappa\left(\left\langle\alpha_{3}, \alpha_{2}\right\rangle\right) e_{\alpha_{1}} e_{\alpha_{2}+\alpha_{3}}+\kappa\left(\left\langle\alpha_{2}, \alpha_{1}\right\rangle\right) \kappa\left(\left\langle\alpha_{3}, \alpha_{1}+\alpha_{2}\right\rangle\right) e_{\alpha_{1}+\alpha_{2}+\alpha_{3}} .
\end{aligned}
$$

Note that the $e_{\beta}$ 's on the right hand side follow the order given in (3.8). Since in the Taylor series expansion on the right hand side of (4.8) $e_{\alpha_{1}+\alpha_{2}+\alpha_{3}}$ is multiplied by $\bar{\Omega}^{-}\left(\alpha_{1}+\alpha_{2}+\alpha_{3}\right)$, by comparing the coefficient of $e_{\alpha_{1}+\alpha_{2}+\alpha_{3}}$ in the left and right hand side of (4.8) we get, from (4.16),

$$
\Delta \bar{\Omega}\left(\alpha_{1}+\alpha_{2}+\alpha_{3}\right)=(-1)^{\alpha_{12}+\alpha_{23}+\alpha_{13}} \alpha_{12}\left(\alpha_{13}+\alpha_{23}\right) \bar{\Omega}^{+}\left(\alpha_{1}\right) \bar{\Omega}^{+}\left(\alpha_{2}\right) \bar{\Omega}^{+}\left(\alpha_{3}\right)+\cdots
$$

where $\cdots$ represent terms other than the one containing the product $\bar{\Omega}^{+}\left(\alpha_{1}\right) \bar{\Omega}^{+}\left(\alpha_{2}\right) \bar{\Omega}^{+}\left(\alpha_{3}\right)$. Happily, this agrees with the result (3.11) from the black hole bound state analysis.

In a similar fashion, let us consider the generic 4-body contribution. We assume the same ordering of the different linear combinations of the $\alpha_{i}$ 's as given in (3.13). Then one finds for the jump across the wall

$$
\begin{aligned}
& \Delta \bar{\Omega}\left(\alpha_{1}+\alpha_{2}+\alpha_{3}+\alpha_{4}\right)=-\bar{\Omega}^{+}\left(\alpha_{1}\right) \bar{\Omega}^{+}\left(\alpha_{2}\right) \bar{\Omega}^{+}\left(\alpha_{3}\right) \bar{\Omega}^{+}\left(\alpha_{4}\right) \times \\
& {\left[\kappa\left(\alpha_{12}\right) \kappa\left(\alpha_{13}+\alpha_{23}\right) \kappa\left(\alpha_{14}+\alpha_{24}+\alpha_{34}\right)\right.} \\
& +\kappa\left(\alpha_{13}\right) \kappa\left(\alpha_{14}+\alpha_{34}\right) \kappa\left(\alpha_{21}+\alpha_{23}+\alpha_{24}\right) \\
& \left.+\kappa\left(\alpha_{23}\right) \kappa\left(\alpha_{14}\right) \kappa\left(\left\langle\alpha_{2}+\alpha_{3}, \alpha_{1}+\alpha_{4}\right\rangle\right)\right]+\ldots \\
& =(-1)^{1+\sum_{i<j} \alpha_{i j}} \bar{\Omega}^{+}\left(\alpha_{1}\right) \bar{\Omega}^{+}\left(\alpha_{2}\right) \bar{\Omega}^{+}\left(\alpha_{3}\right) \bar{\Omega}^{+}\left(\alpha_{4}\right) \times \\
& {\left[\alpha_{12} \alpha_{13} \alpha_{24}+\alpha_{13} \alpha_{14} \alpha_{24}+\alpha_{12} \alpha_{23} \alpha_{24}+\alpha_{14} \alpha_{23} \alpha_{24}\right.} \\
& \left.+\alpha_{12} \alpha_{23} \alpha_{34}+\alpha_{13} \alpha_{23} \alpha_{34}+\alpha_{14} \alpha_{23} \alpha_{34}+\alpha_{13} \alpha_{24} \alpha_{34}\right]+\ldots,
\end{aligned}
$$

where the dots represent other contributions. This again agrees with the result (3.15) of the black hole bound state analysis. For completeness also give the result for the two body contribution to $\Delta \bar{\Omega}$ :

$$
\Delta \bar{\Omega}\left(\alpha_{1}+\alpha_{2}\right)=(-1)^{\alpha_{12}+1} \alpha_{12} \bar{\Omega}^{+}\left(\alpha_{1}\right) \bar{\Omega}^{+}\left(\alpha_{2}\right)+\cdots .
$$

Finally note that this method can be easily generalized to the case when some of the $\alpha_{i}$ 's are equal. For example if we are looking for a term $\prod_{i}\left(\bar{\Omega}^{+}\left(\alpha_{i}\right)\right)^{m_{i}}$ in the expression 
for $\bar{\Omega}^{-}\left(\sum_{i} m_{i} \alpha_{i}\right)$, then we must expand the left hand side of (4.8) so that in $V_{\alpha_{i}}$ we keep the $\left(\bar{\Omega}^{+}\left(\alpha_{i}\right) e_{\alpha_{i}}\right)^{m_{i}} / m_{i}$ ! term, then carry out the rearrangement described above, and finally identify the coefficient of $e_{\sum_{i} m_{i} \alpha_{i}}$ in the resulting expression. Alternatively we could simply use the generic $n$-body formula for non-identical particles, take the limit when several of the $\alpha_{i}$ 's approach each other and then include the symmetry factor $1 / \prod_{i} m_{i}$ ! in accordance with (2.16). It is easy to see how this rule arises from the KS formula, - given a factor of $\left(e_{\beta}\right)^{m}$ for any vector $\beta$ we can replace it by $e_{\beta^{(1)}} e_{\beta^{(2)}} \cdots e_{\beta^{(m)}}$ for $m$ distinct vectors $\beta^{(1)}, \ldots, \beta^{(m)}$ with slightly different phases, ${ }^{15}$ carry out the abovementioned manipulations for rearranging the vectors and then take the limit when all the $\beta^{(i)}$ 's approach each other to recover the desired result. There is a priori an ambiguity in this procedure since in the final configuration the relative ordering between two vectors which only differ by the replacement of $\beta^{(i)}$ by $\beta^{(j)}$ for some pair $(i, j)$ is arbitrary, but this does not affect the final result since changing this relative order picks up a commutator factor that vanishes as $\beta^{(i)} \rightarrow \beta^{(j)}$. Thus the only effect of having identical particles will be the Boltzmann symmetry factor $1 / m$ !.

\subsection{Semi-primitive wall-crossing formulæ and generalizations}

A general wall-crossing formula involves computing $\Omega^{-}\left(m \gamma_{1}+n \gamma_{2}\right)$ in terms of $\Omega^{+}\left(k \gamma_{1}+\ell \gamma_{2}\right)$ for $k \leq m, \ell \leq n$. We define the order of the wall-crossing formula as the smaller of $m$ and $n$. In this and the following subsections we give wall-crossing formulæ for increasing order, starting with order one in this section and ending at order three. For simplicity we shall give the result for the case when $m$ is fixed to be 1,2 or 3 , but the result can be easily generalized to the case when $n$ is 1,2 or 3 (see $\S 4.6$ ). In the D6-D0 example described in appendix $\mathrm{B}$, order corresponds to the number of D6-branes or the rank of the sheaf.

\subsubsection{Order one}

To extract the semi-primitive wall-crossing formula from the KS formula, we project (4.7), (4.8) to the algebra $\mathcal{A}_{1, \infty}$ : Thus we have

$$
A_{\gamma_{1}, \gamma_{2}}=V_{\gamma_{1}}^{+} V_{\gamma_{1}+\gamma_{2}}^{+} V_{\gamma_{1}+2 \gamma_{2}}^{+} \cdots V_{\gamma_{2}}^{+}=V_{\gamma_{2}}^{-} \cdots V_{\gamma_{1}+2 \gamma_{2}}^{-} V_{\gamma_{1}+\gamma_{2}}^{-} V_{\gamma_{1}}^{-} .
$$

Noting that $e_{\gamma_{1}+N \gamma_{2}}$ all commute in $\mathcal{A}_{1, \infty}$, this can be rewritten as

$$
A_{\gamma_{1}, \gamma_{2}}=e^{X_{1}^{+}} e^{Y}=e^{Y} e^{X_{1}^{-}}
$$

where

$$
X_{1}^{ \pm}=\sum_{N=0}^{\infty} \bar{\Omega}^{ \pm}\left(\gamma_{1}+N \gamma_{2}\right) e_{\gamma_{1}+N \gamma_{2}}, \quad Y=\sum_{\ell=1}^{\infty} \bar{\Omega}^{+}\left(\ell \gamma_{2}\right) e_{\ell \gamma_{2}}=\sum_{\ell=1}^{\infty} \bar{\Omega}^{-}\left(\ell \gamma_{2}\right) e_{\ell \gamma_{2}} .
$$

It follows from (4.21) that

$$
X_{1}^{-}=e^{-Y} X_{1}^{+} e^{Y} .
$$

\footnotetext{
${ }^{15}$ For this manipulation we can ignore the fact that the charges are allowed to take values on a lattice.
} 
To evaluate the right hand side of (4.23) we first observe that, for a single term in $Y$,

$$
e^{-\bar{\Omega}^{+}\left(\ell \gamma_{2}\right) e_{\ell \gamma_{2}}} e_{\gamma_{1}+N \gamma_{2}} e^{\bar{\Omega}^{+}\left(\ell \gamma_{2}\right) e_{\ell \gamma_{2}}}=\sum_{n_{\ell}=0}^{\infty} \frac{1}{n_{\ell} !}\left[\kappa\left(\ell \gamma_{12}\right) \bar{\Omega}^{+}\left(\ell \gamma_{2}\right)\right]^{n_{\ell}} e_{\gamma_{1}+\left(N+\ell n_{\ell}\right) \gamma_{2}} .
$$

Thus,

$$
e^{-Y} e_{\gamma_{1}+N \gamma_{2}} e^{Y}=\sum_{\left\{n_{k}\right\}}\left(\prod_{\ell} \frac{\left[\kappa\left(\ell \gamma_{12}\right) \bar{\Omega}^{+}\left(\ell \gamma_{2}\right)\right]^{n_{\ell}}}{n_{\ell} !}\right) e_{\gamma_{1}+\left(N+\sum_{\ell} \ell n_{\ell}\right) \gamma_{2}},
$$

and from eq, (4.23),

$$
X_{1}^{-}=\sum_{N=0}^{\infty} \bar{\Omega}^{+}\left(\gamma_{1}+N \gamma_{2}\right) \sum_{\left\{n_{k}\right\}}\left(\prod_{\ell} \frac{\left[\kappa\left(\ell \gamma_{12}\right) \bar{\Omega}^{+}\left(\ell \gamma_{2}\right)\right]^{n_{\ell}}}{n_{\ell} !}\right) e_{\gamma_{1}+\left(N+\sum_{\ell} \ell n_{\ell}\right) \gamma_{2}} .
$$

Using the relation between $X_{1}^{-}$and $\bar{\Omega}^{-}\left(\gamma_{1}+N \gamma_{2}\right)$ given in (4.22) we now get

$$
\bar{\Omega}^{-}\left(\gamma_{1}+N \gamma_{2}\right)=\sum_{N^{\prime}=0}^{N} \bar{\Omega}^{+}\left(\gamma_{1}+N^{\prime} \gamma_{2}\right) \Omega_{\mathrm{halo}}\left(\gamma_{1}, N-N^{\prime}\right)
$$

where we defined

$$
\Omega_{\text {halo }}\left(\gamma_{1}, N\right)=\sum_{\substack{\left\{n_{k}\right\} \\ \sum k n_{k}=N}} \prod_{\ell} \frac{\left[\kappa\left(\ell \gamma_{12}\right) \bar{\Omega}^{+}\left(\ell \gamma_{2}\right)\right]^{n_{\ell}}}{n_{\ell} !}
$$

In terms of the partition function $Z^{ \pm}(1, q)=\bar{Z}^{ \pm}(1, q)$ defined in (4.11), we obtain

$$
Z^{-}(1, q)=Z^{+}(1, q) Z_{\text {halo }}\left(\gamma_{1}, q\right)
$$

where

$$
Z_{\text {halo }}\left(\gamma_{1}, q\right)=\sum_{N=0}^{\infty} \Omega_{\text {halo }}\left(\gamma_{1}, N\right) q^{N}=\exp \left(\sum_{\ell=1}^{\infty}(-1)^{\left\langle\gamma_{1}, \ell \gamma_{2}\right\rangle}\left\langle\gamma_{1}, \ell \gamma_{2}\right\rangle \bar{\Omega}^{+}\left(\ell \gamma_{2}\right) q^{\ell}\right)
$$

reproducing $(2.22),(2.23)$.

\subsubsection{Order two}

We now extend the semi-primitive wall-crossing formula to order 2, i.e. compute $Z^{-}(2, q)$. To this aim we project $(4.7),(4.8)$ to the algebra $\mathcal{A}_{2, \infty}$,

$$
A_{\gamma_{1}, \gamma_{2}}=\left[\prod_{k=0}^{\infty} V_{\gamma_{1}+k \gamma_{2}}^{+} V_{2 \gamma_{1}+(2 k+1) \gamma_{2}}^{+}\right] \cdot V_{\gamma_{2}}^{+}=V_{\gamma_{2}}^{-} \cdot\left[\prod_{k=\infty}^{0} V_{2 \gamma_{1}+(2 k+1) \gamma_{2}}^{-} V_{\gamma_{1}+k \gamma_{2}}^{-}\right] \text {. }
$$

We can combine all factors of $V_{\gamma_{1}+N \gamma_{2}}^{ \pm}$and $V_{2 \gamma_{1}+N \gamma_{2}}^{ \pm}$on either side into a single exponential, by using the level two truncation (4.13) of the $\mathrm{BCH}$ formula. Thus, we now have

$$
A_{\gamma_{1}, \gamma_{2}}=e^{X_{1}^{+}+X_{2}^{+}} e^{Y}=e^{Y} e^{X_{1}^{-}+X_{2}^{-}}
$$


where $X_{1}^{ \pm}, Y$ are the same as in (4.22), while

$$
\begin{aligned}
X_{2}^{ \pm} & =\sum_{N=0}^{\infty} \widetilde{\Omega}_{2}^{ \pm}(N) e_{2 \gamma_{1}+N \gamma_{2}} \\
\widetilde{\Omega}_{2}^{ \pm}(N) & \equiv \bar{\Omega}^{ \pm}\left(2 \gamma_{1}+N \gamma_{2}\right) \pm \frac{1}{4} \sum_{i+j=N} \kappa\left(|j-i| \gamma_{12}\right) \bar{\Omega}^{ \pm}\left(\gamma_{1}+i \gamma_{2}\right) \bar{\Omega}^{ \pm}\left(\gamma_{1}+j \gamma_{2}\right) .
\end{aligned}
$$

eq. (4.32) now implies that

$$
X_{1}^{-}=e^{-Y} X_{1}^{+} e^{Y} \quad X_{2}^{-}=e^{-Y} X_{2}^{+} e^{Y} .
$$

Using the analog of (4.27) with $\gamma_{1} \rightarrow 2 \gamma_{1}$ and eq, (4.33) we get

$$
\widetilde{\Omega}_{2}^{-}(N)=\sum_{N^{\prime}=0}^{N} \widetilde{\Omega}_{2}^{+}\left(N^{\prime}\right) \Omega_{\text {halo }}\left(2 \gamma_{1}, N-N^{\prime}\right),
$$

where $\Omega_{\text {halo }}$ is defined in (4.28). Combining this with (4.27), (4.34), we arrive at

$$
\begin{aligned}
& \bar{\Omega}^{-}\left(2 \gamma_{1}+N \gamma_{2}\right)=\sum_{0 \leq N^{\prime} \leq N} \bar{\Omega}^{+}\left(2 \gamma_{1}+N^{\prime} \gamma_{2}\right) \Omega_{\text {halo }}\left(2 \gamma_{1}, N-N^{\prime}\right) \\
& +\frac{1}{2} \sum_{0 \leq i<j ; i+j \leq N} \kappa\left((j-i) \gamma_{12}\right) \bar{\Omega}^{+}\left(\gamma_{1}+i \gamma_{2}\right) \bar{\Omega}^{+}\left(\gamma_{1}+j \gamma_{2}\right) \Omega_{\text {halo }}\left(2 \gamma_{1}, N-i-j\right) \\
& \quad-\frac{1}{2} \sum_{\substack{0 \leq j<i, i+j=N \\
0 \leq i^{\prime} \leq i, 0 \leq j^{\prime} \leq j}} \kappa\left((j-i) \gamma_{12}\right) \bar{\Omega}^{+}\left(\gamma_{1}+i^{\prime} \gamma_{2}\right) \bar{\Omega}^{+}\left(\gamma_{1}+j^{\prime} \gamma_{2}\right) \Omega_{\text {halo }}\left(\gamma_{1}, i-i^{\prime}\right) \Omega_{\text {halo }}\left(\gamma_{1}, j-j^{\prime}\right) .
\end{aligned}
$$

This result generalizes eq. (4.10) in [44].

The partition functions (4.11) for $M=2$ are most conveniently expressed in terms of "modified partition functions"

$$
\widetilde{Z}_{2}^{ \pm}(q)=\sum_{N=0}^{\infty} \widetilde{\Omega}_{2}^{ \pm}(N) q^{N}
$$

as

$$
\bar{Z}^{ \pm}(2, q)=\widetilde{Z}_{2}^{ \pm}(q) \pm \frac{1}{2} \bar{Z}^{ \pm}(1, q) \star \bar{Z}^{ \pm}(1, q),
$$

where the star product is defined by

$$
\sum_{i=0}^{\infty} a_{i} q^{i} \star \sum_{i=0}^{\infty} b_{j} q^{j}=\sum_{0 \leq i<j} \kappa\left((i-j) \gamma_{12}\right) a_{i} b_{j} q^{i+j} .
$$

The relation (4.36) then simplifies to a simple wall-crossing identity for the modified partition functions,

$$
\widetilde{Z}_{2}^{-}(q)=\widetilde{Z}_{2}^{+}(q) Z_{\text {halo }}\left(2 \gamma_{1}, q\right)
$$

where $Z_{\text {halo }}\left(2 \gamma_{1}, q\right)$ is given by the same formula as in (4.30) with $\gamma_{1} \rightarrow 2 \gamma_{1}$. Thus, the effective description is still in terms of a halo of non-interacting Boltzmannian particles around a core with effective degeneracy $\widetilde{\Omega}_{2}^{ \pm}(N)$. 


\subsubsection{Order three}

The projection of (4.7), (4.8) to the algebra $\mathcal{A}_{3, \infty}$ reads

$$
\begin{aligned}
A_{\gamma_{1}, \gamma_{2}} & =\left[\prod_{k=0}^{\infty} V_{\gamma_{1}+k \gamma_{2}}^{+} V_{3 \gamma_{1}+(3 k+1) \gamma_{2}}^{+} V_{2 \gamma_{1}+(2 k+1) \gamma_{2}}^{+} V_{3 \gamma_{1}+(3 k+2) \gamma_{2}}^{+}\right] \cdot V_{\gamma_{2}}^{+} \\
& =V_{\gamma_{2}}^{-} \cdot\left[\prod_{k=\infty}^{0} V_{3 \gamma_{1}+(3 k+2) \gamma_{2}}^{-} V_{2 \gamma_{1}+(2 k+1) \gamma_{2}}^{-} V_{3 \gamma_{1}+(3 k+1) \gamma_{2}}^{-} V_{\gamma_{1}+k \gamma_{2}}^{-}\right] .
\end{aligned}
$$

We can combine factors according to the $\gamma_{1}$-charge by using the level three truncation of the BCH formula:

$$
\begin{aligned}
\log \left(e^{X_{0}} e^{X_{1}} \ldots e^{X_{N}}\right)= & \sum_{i=0}^{N} X_{i}+\frac{1}{2} \sum_{0 \leq i<j \leq N}\left[X_{i}, X_{j}\right]+\frac{1}{12} \sum_{i, j=0}^{N}\left[X_{i},\left[X_{i}, X_{j}\right]\right] \\
& +\frac{1}{6}\left(\sum_{0 \leq i<j<k \leq N}+\sum_{0 \leq k<j<i \leq N}\right)\left[X_{i},\left[X_{j}, X_{k}\right]\right]+\ldots
\end{aligned}
$$

Thus, we now have

$$
A_{\gamma_{1}, \gamma_{2}}=e^{X_{1}^{+}+X_{2}^{+}+X_{3}^{+}} e^{Y}=e^{Y} e^{X_{1}^{-}+X_{2}^{-}+X_{3}^{-}} .
$$

$X_{2}^{ \pm}, X_{1}^{ \pm}, Y$ are the same as in (4.33), (4.22), (4.21) while

$$
X_{3}^{ \pm}=\sum_{N=0}^{\infty} \widetilde{\Omega}_{3}^{ \pm}\left(3 \gamma_{1}+N \gamma_{2}\right) e_{3 \gamma_{1}+N \gamma_{2}}
$$

where we defined

$$
\begin{aligned}
\widetilde{\Omega}_{3}^{ \pm}(N)= & \bar{\Omega}^{ \pm}\left(3 \gamma_{1}+N \gamma_{2}\right) \\
& \pm \frac{1}{2} \sum_{i+j=N} \kappa\left(|j-2 i| \gamma_{12}\right) \bar{\Omega}^{ \pm}\left(\gamma_{1}+i \gamma_{2}\right) \bar{\Omega}^{ \pm}\left(2 \gamma_{1}+j \gamma_{2}\right) \\
& +\frac{1}{12} \sum_{2 i+j=N}\left[\kappa\left((j-i) \gamma_{12}\right)\right]^{2}\left[\bar{\Omega}^{ \pm}\left(\gamma_{1}+i \gamma_{2}\right)\right]^{2} \bar{\Omega}^{ \pm}\left(\gamma_{1}+j \gamma_{2}\right) \\
& +\sum_{\substack{0 \leq i<j<k \\
i+j+k=N}} \kappa(i, j, k) \bar{\Omega}^{ \pm}\left(\gamma_{1}+i \gamma_{2}\right) \bar{\Omega}^{ \pm}\left(\gamma_{1}+j \gamma_{2}\right) \bar{\Omega}^{ \pm}\left(\gamma_{1}+k \gamma_{2}\right) \\
\kappa(i, j, k) \equiv & \frac{1}{6} \kappa\left((i-j) \gamma_{12}\right) \kappa\left((i+j-2 k) \gamma_{12}\right)+\frac{1}{6} \kappa\left((k-j) \gamma_{12}\right) \kappa\left((j+k-2 i) \gamma_{12}\right)
\end{aligned}
$$

Note that (4.47) may be written differently using the Jacobi identity

$$
\begin{aligned}
& -\kappa\left((j-i) \gamma_{12}\right) \kappa\left((i+j-2 k) \gamma_{12}\right) \\
& -\kappa\left((k-j) \gamma_{12}\right) \\
& \quad \kappa\left((j+k-2 i) \gamma_{12}\right) \\
& +\kappa\left((k-i) \gamma_{12}\right) \kappa\left((i+k-2 j) \gamma_{12}\right)=0
\end{aligned}
$$


and that $\kappa(i, j, k)$ is symmetric under exchange $i \leftrightarrow k$. As before, eq, (4.44), besides leading to eqs.(4.23) and (4.35), gives

$$
X_{3}^{-}=e^{-Y} X_{3}^{+} e^{Y} .
$$

Using the analog of (4.27) with $\gamma_{1} \rightarrow 3 \gamma_{1}$ and eqs.(4.45) we get

$$
\tilde{\Omega}_{3}^{-}(N)=\sum_{0 \leq N^{\prime} \leq N} \tilde{\Omega}_{3}^{+}\left(N^{\prime}\right) \Omega_{\text {halo }}\left(3 \gamma_{1}, N-N^{\prime}\right)
$$

where $\Omega_{\text {halo }}$ is defined in (4.28). Putting together (4.46) and (4.50), we arrive at

$$
\begin{aligned}
& \bar{\Omega}^{-}\left(3 \gamma_{1}+N \gamma_{2}\right)=\sum_{N^{\prime}} \bar{\Omega}^{+}\left(3 \gamma_{1}+N^{\prime} \gamma_{2}\right) \Omega_{\text {halo }}\left(3 \gamma_{1}, N-N^{\prime}\right) \\
& +\frac{1}{2} \sum_{\substack{i \geq 0, j \geq 0 \\
i+j \leq N}} \kappa\left(|j-2 i| \gamma_{12}\right) \mid \bar{\Omega}^{+}\left(\gamma_{1}+i \gamma_{2}\right) \bar{\Omega}^{+}\left(2 \gamma_{1}+j \gamma_{2}\right) \Omega_{\text {halo }}\left(3 \gamma_{1}, N-i-j\right) \\
& +\frac{1}{12} \sum_{\substack{i \geq 0, j \geq 0 \\
2 i+j \leq N}}\left[\kappa\left((j-i) \gamma_{12}\right)\right]^{2}\left[\bar{\Omega}^{+}\left(\gamma_{1}+i \gamma_{2}\right)\right]^{2} \bar{\Omega}^{+}\left(\gamma_{1}+j \gamma_{2}\right) \Omega_{\text {halo }}\left(3 \gamma_{1}, N-2 i-j\right) \\
& +\sum_{\substack{0 \leq i<j<k \\
i+j+k \leq N}} \kappa(i, j, k) \bar{\Omega}^{+}\left(\gamma_{1}+i \gamma_{2}\right) \bar{\Omega}^{+}\left(\gamma_{1}+j \gamma_{2}\right) \bar{\Omega}^{+}\left(\gamma_{1}+k \gamma_{2}\right) \Omega_{\text {halo }}\left(3 \gamma_{1}, N-i-j-k\right), \\
& +\frac{1}{2} \sum_{\substack{i+j=N \\
0 \leq i^{\prime} \leq i, 0 \leq j^{\prime} \leq j}} \kappa\left(|j-2 i| \gamma_{12}\right) \bar{\Omega}^{+}\left(\gamma_{1}+i^{\prime} \gamma_{2}\right) \bar{\Omega}^{+}\left(2 \gamma_{1}+j^{\prime} \gamma_{2}\right) \Omega_{\text {halo }}\left(\gamma_{1}, i-i^{\prime}\right) \Omega_{\text {halo }}\left(2 \gamma_{1}, j-j^{\prime}\right) \\
& +\frac{1}{8} \sum_{\substack{i+j=N \\
0 \leq i^{\prime} \leq i ; k+l \leq j}} \kappa\left(|j-2 i| \gamma_{12}\right) \kappa\left(|l-k| \gamma_{12}\right) \bar{\Omega}^{+}\left(\gamma_{1}+i^{\prime} \gamma_{2}\right) \bar{\Omega}^{+}\left(\gamma_{1}+k \gamma_{2}\right) \bar{\Omega}^{+}\left(\gamma_{1}+l \gamma_{2}\right) \\
& \Omega_{\text {halo }}\left(\gamma_{1}, i-i^{\prime}\right) \Omega_{\text {halo }}\left(2 \gamma_{1}, j-k-l\right) \\
& -\frac{1}{12} \sum_{\substack{2 i+j=N \\
0 \leq i^{\prime}, i^{\prime \prime} \leq i, 0 \leq j^{\prime} \leq j}}\left[\kappa\left((j-i) \gamma_{12}\right)\right]^{2} \bar{\Omega}^{+}\left(\gamma_{1}+i^{\prime} \gamma_{2}\right) \bar{\Omega}^{+}\left(\gamma_{1}+i^{\prime \prime} \gamma_{2}\right) \bar{\Omega}^{+}\left(\gamma_{1}+j^{\prime} \gamma_{2}\right) \\
& \Omega_{\text {halo }}\left(\gamma_{1}, i-i^{\prime}\right) \Omega_{\text {halo }}\left(\gamma_{1}, i-i^{\prime \prime}\right) \Omega_{\text {halo }}\left(\gamma_{1}, j-j^{\prime}\right) \\
& -\sum_{\substack{i+j+k=N ; i>j>k>0 \\
0 \leq i^{\prime} \leq i ; 0 \leq j^{\prime} \leq j ; 0 \leq k^{\prime} \leq k}} \kappa(i, j, k) \bar{\Omega}^{+}\left(\gamma_{1}+i^{\prime} \gamma_{2}\right) \bar{\Omega}^{+}\left(\gamma_{1}+j^{\prime} \gamma_{2}\right) \bar{\Omega}^{+}\left(\gamma_{1}+k^{\prime} \gamma_{2}\right) \\
& \Omega_{\text {halo }}\left(\gamma_{1}, i-i^{\prime}\right) \Omega_{\text {halo }}\left(\gamma_{1}, j-j^{\prime}\right) \Omega_{\text {halo }}\left(\gamma_{1}, k-k^{\prime}\right) \\
& +\frac{1}{8} \sum_{\substack{i+j+k=N \\
0 \leq i^{\prime} \leq i, 0 \leq j^{\prime} \leq j, 0 \leq k^{\prime} \leq k}} \kappa\left(|j+k-2 i| \gamma_{12}\right) \kappa\left(|j-k| \gamma_{12}\right) \bar{\Omega}^{+}\left(\gamma_{1}+i^{\prime} \gamma_{2}\right) \bar{\Omega}^{+}\left(\gamma_{1}+k^{\prime} \gamma_{2}\right) \bar{\Omega}^{+}\left(\gamma_{1}+j^{\prime} \gamma_{2}\right) \\
& \Omega_{\text {halo }}\left(\gamma_{1}, i-i^{\prime}\right) \Omega_{\text {halo }}\left(\gamma_{1}, j-j^{\prime}\right) \Omega_{\text {halo }}\left(\gamma_{1}, k-k^{\prime}\right) .
\end{aligned}
$$

As before, we may introduce the "modified partition functions"

$$
\tilde{Z}_{3}^{ \pm}(q)=\sum_{N=0}^{\infty} \tilde{\Omega}_{3}^{ \pm}(N) q^{N},
$$


in terms of which the wall-crossing relation takes the simple form

$$
\tilde{Z}_{3}^{-}(q)=\tilde{Z}_{3}^{+}(q) Z_{\text {halo }}\left(3 \gamma_{1}, q\right),
$$

where $Z_{\text {halo }}\left(3 \gamma_{1}, q\right)$ is defined in (4.30). The modified partition functions are related to the original partition functions $\bar{Z}^{ \pm}(3, q)$ by a relation of the form

$$
\tilde{Z}_{3}^{ \pm}(q)=\bar{Z}^{ \pm}(3, q)+\bar{Z}^{ \pm}(1, q) \star_{ \pm} \bar{Z}^{ \pm}(2, q)+\bar{Z}^{ \pm}(1, q) \star_{ \pm} \bar{Z}^{ \pm}(1, q) \star_{ \pm} \bar{Z}^{ \pm}(1, q)
$$

with the definitions of the 'star' products following from (4.46).

\section{6 $\quad \gamma_{12}>0$ case}

We shall now briefly discuss what happens when $\gamma_{12}>0$. We can of course use all the formulæ derived in this section with $\gamma_{1} \leftrightarrow \gamma_{2}$, but then e.g. eq. (4.51) will give the wallcrossing formula in the charge sector $N \gamma_{1}+3 \gamma_{2}$. So if we want to find the wall-crossing formula for $3 \gamma_{1}+N \gamma_{2}$ we cannot get the result by exchanging $\gamma_{1}$ and $\gamma_{2}$ in (4.51). Instead we keep $\gamma_{12}>0$ and carefully examine how the subsequent equations are affected. It is easy to see that the only place where the sign of $\gamma_{12}$ enters is in eq, (2.6); for $\gamma_{12}>0$ the $<\operatorname{sign}$ in $(2.6)$ is replaced by a $>$ sign. So if we continue to define $\Omega^{ \pm}$as in $\S 2.2$ then $\Omega^{+}$will denote the index in the chamber in which the multi-centered bound states of black hole molecules exist. We would however like to define $\Omega^{+}$as the index associated with single black hole molecules, and for this we exchange the definitions of $\Omega^{+}$and $\Omega^{-}$. Thus for example in eq, (4.51) we have to now exchange $\Omega^{+}$with $\Omega^{-}$so that we have an expression for $\Omega^{+}$in terms of $\Omega^{-}$. We can in principle solve these equations iteratively to find $\Omega^{-}$in terms of $\Omega^{+}$, but we shall now suggest a simpler method. For this note that exchanging $\Omega^{+}$and $\Omega^{-}$in the wall-crossing formula (4.8) is equivalent to changing the order of the products on both sides of (4.8). This in turn is equivalent to keeping the same order as in (4.8) but changing the sign of all the structure constants in the algebra (4.1). This can be achieved by changing $\gamma_{12}$ to $-\gamma_{12}$. Thus if we replace $\gamma_{12}$ by $-\left|\gamma_{12}\right|$ in the formulæ we have derived (e.g. (4.51)), we shall get the correct wall-crossing formula for both signs of $\gamma_{12}$.

\subsection{Refined wall-crossing and motivic invariants}

We have already introduced the refined invariants in $\S 2.6$. In this subsection we shall review the KS motivic wall-crossing formula that computes the jump in the refined index across walls of marginal stability and compare it with our wall-crossing formula based on the analysis of supergravity bound states.

In order to describe the motivic generalization of the wall-crossing formula [11], we consider a set of generators $\left\{\hat{e}_{\gamma}, \gamma \in \Gamma\right\}$ satisfying the quantum torus relations

$$
\hat{e}_{\gamma} \hat{e}_{\gamma^{\prime}}=(-y)^{\left\langle\gamma, \gamma^{\prime}\right\rangle} \hat{e}_{\gamma+\gamma^{\prime}} .
$$

The associated Lie algebra is

$$
\left[\hat{e}_{\gamma}, \hat{e}_{\gamma^{\prime}}\right]=\left((-y)^{\left\langle\gamma, \gamma^{\prime}\right\rangle}-(-y)^{-\left\langle\gamma, \gamma^{\prime}\right\rangle}\right) \hat{e}_{\gamma+\gamma^{\prime}} .
$$


Let us also introduce the quantum dilogarithm,

$$
\mathbf{E}(x)=\sum_{n=0}^{\infty} \frac{(x y)^{n}}{\left(1-y^{2}\right) \ldots\left(1-y^{2 n}\right)}=\prod_{n=0}^{\infty}\left(1+(-y)^{2 n+1} x\right)^{-1} .
$$

This satisfies the pentagon identity

$$
\mathbf{E}\left(x_{1}\right) \mathbf{E}\left(x_{2}\right)=\mathbf{E}\left(x_{2}\right) \mathbf{E}\left(x_{12}\right) \mathbf{E}\left(x_{1}\right)
$$

where $x_{1}, x_{2}$ are two non-commutative variables satisfying $x_{1} x_{2} / y=y x_{2} x_{1} \equiv-x_{12}$, and reduces to the ordinary dilogarithm for $\log y \equiv \nu \rightarrow 0$,

$$
\mathbf{E}(x)=\exp \left(-\frac{1}{2 \nu} \operatorname{Li}_{2}(x)+\frac{x}{12(1-x)} \nu+\frac{7 x(1+x)}{720(x-1)^{3}} \nu^{3}+\ldots\right) .
$$

We attach to the charge vector $\gamma$ the generator ${ }^{16}$

$$
\hat{U}_{\gamma}=\prod_{n \in \mathbb{Z}} \mathbf{E}\left(y^{n} \hat{e}_{\gamma}\right)^{-(-1)^{n} \Omega_{\mathrm{ref}, n}(\gamma)} .
$$

The motivic version of the KS wall-crossing formula [11, 34, 35] again states that the product

$$
\hat{A}_{\gamma_{1}, \gamma_{2}}=\prod_{\substack{\gamma=M \gamma_{1}+N \gamma_{2}, M \geq 0, N \geq 0}} \hat{U}_{\gamma}
$$

ordered so that as we move from left to right the corresponding $Z_{\gamma}$ 's are ordered clockwise, stays constant across the hyperplane of marginal stability $\mathcal{P}\left(\gamma_{1}, \gamma_{2}\right)$.

As in the classical case, it is advantageous to combine the generators $\hat{U}_{k \gamma}$ for $k \geq 1$ in a single factor $\hat{V}_{\gamma}$. For this purpose, rewrite the operator $\hat{U}_{\gamma}$, using the identity for the quantum dilogarithm

$$
\mathbf{E}(x)=\exp \left(\sum_{k=1}^{\infty} \frac{(x y)^{k}}{k\left(1-y^{2 k}\right)}\right) \text {, }
$$

as

$$
\hat{U}_{\gamma}=\exp \left(\sum_{k=1}^{\infty} \frac{\Omega_{\mathrm{ref}}\left(\gamma, y^{k}\right)}{k\left(y^{k}-y^{-k}\right)} \hat{e}_{k \gamma}\right) .
$$

Then the product of $\hat{U}_{\ell \gamma}$ over all $\ell \geq 1, \gamma$ being a primitive vector, can be written in terms of $\bar{\Omega}_{\text {ref }}(\gamma, y)$ defined in (2.38):

$$
\hat{V}_{\gamma}=\prod_{\ell \geq 1} \hat{U}_{\ell \gamma}=\exp \left(\sum_{N=1}^{\infty} \bar{\Omega}_{\mathrm{ref}}(N \gamma, y) \tilde{e}_{N \gamma}\right), \quad \tilde{e}_{\gamma} \equiv \frac{\hat{e}_{\gamma}}{y-y^{-1}} .
$$

This is the motivic generalization of (4.6). The wall-crossing formula now takes the form

$$
\prod_{\substack{M \geq 0, N \geq 0>0, \operatorname{gcd}(M, N)=1, M / N \downarrow}} \hat{V}_{M \gamma_{1}+N \gamma_{2}}^{+}=\prod_{\substack{M \geq 0, N \geq 0>0, \operatorname{gcd}(M, N)=1, M / N \uparrow}} \hat{V}_{M \gamma_{1}+N \gamma_{2}}^{-},
$$

\footnotetext{
${ }^{16}$ In supersymmetric gauge theories, for a vector multiplet with unit degeneracy, $\hat{U}_{\gamma}$ reduces to $\hat{U}_{\gamma}=$ $\mathbf{E}\left(y \hat{e}_{\gamma}\right) \mathbf{E}\left(y^{-1} \hat{e}_{\gamma}\right)$ while for a hypermultiplet one has $\hat{U}_{\gamma}=\mathbf{E}\left(e_{\gamma}\right)^{-2}$.
} 
where $\hat{V}^{ \pm}$are computed using the $\bar{\Omega}_{\text {ref }}(\gamma, y)$ in the chambers $c^{ \pm}$. It follows from (4.56), (4.66), (4.64) and (4.65) that expressed in terms of $\bar{\Omega}_{\text {ref }}(\gamma, y)$ the wall-crossing formula will satisfy manifest 'charge conservation laws'.

To see how this reduces to the classical KS formula (4.8) in the limit $y \rightarrow 1$, note that the generators

$$
e_{\gamma}=\lim _{y \rightarrow 1} \tilde{e}_{\gamma}, \quad \tilde{e}_{\gamma} \equiv \frac{\hat{e}_{\gamma}}{y-y^{-1}}
$$

satisfy the commutation relations (4.1). Moreover in this limit $\Omega_{\mathrm{ref}}(\gamma, y)$ approaches $\Omega(\gamma)$. Thus $\hat{V}^{ \pm}$defined in (4.64) reduce to $V^{ \pm}$and we recover (4.8).

\subsection{Semi-primitive refined wall-crossings and its generalizations}

The rescaled generators $\tilde{e}_{\gamma}=\hat{e}_{\gamma} /\left(y-y^{-1}\right)$ satisfy the same Lie algebra as (4.1),

$$
\left[\tilde{e}_{\gamma}, \tilde{e}_{\gamma^{\prime}}\right]=\kappa\left(\left\langle\gamma, \gamma^{\prime}\right\rangle, y\right) \tilde{e}_{\gamma+\gamma^{\prime}}
$$

provided $\kappa(x)$ is replaced by its quantum deformation

$$
\kappa(x, y) \equiv \frac{(-y)^{x}-(-y)^{-x}}{y-1 / y}=(-1)^{x} \sinh (\nu x) / \sinh \nu, \quad \nu \equiv \ln y .
$$

Moreover, the generators $\hat{V}_{\gamma}$ in (4.64) can be obtained from their classical counterpart (4.6) by replacing $\bar{\Omega}(\gamma) \mapsto \bar{\Omega}_{\text {ref }}(\gamma, y), e_{\gamma} \mapsto \tilde{e}_{\gamma}$. Therefore, the wall-crossing formulæ derived in section 4 and appendix A carry over to the motivic case by just replacing

$$
\Omega(\gamma) \mapsto \Omega_{\mathrm{ref}}(\gamma, y), \quad \bar{\Omega}(\gamma) \mapsto \bar{\Omega}_{\mathrm{ref}}(\gamma, y), \quad \kappa(x) \mapsto \kappa(x, y) .
$$

In particular, the primitive wall-crossing formula takes the form [16, 34]

$$
\Delta \Omega_{\mathrm{ref}}\left(\gamma \rightarrow \gamma_{1}+\gamma_{2}, y\right)=\frac{(-y)^{\left\langle\gamma_{1}, \gamma_{2}\right\rangle}-(-y)^{-\left\langle\gamma_{1}, \gamma_{2}\right\rangle}}{y-1 / y} \Omega_{\mathrm{ref}}\left(\gamma_{1}, y\right) \Omega_{\mathrm{ref}}\left(\gamma_{2}, y\right)
$$

while the refined semi-primitive wall-crossing formula is given by

$$
Z^{-}(1, q, y)=Z^{+}(1, q, y) Z_{\text {halo }}\left(\gamma_{1}, q, y\right)
$$

where

$$
Z_{\text {halo }}\left(\gamma_{1}, q, y\right) \equiv \exp \left(\sum_{\ell=1}^{\infty} \frac{(-y)^{\left\langle\gamma_{1}, \ell \gamma_{2}\right\rangle}-(-y)^{-\left\langle\gamma_{1}, \ell \gamma_{2}\right\rangle}}{y-y^{-1}} \bar{\Omega}_{\mathrm{ref}}\left(\ell \gamma_{2}, y\right) q^{\ell}\right)
$$

On the right hand side $\bar{\Omega}_{\text {ref }}\left(\ell \gamma_{2}, y\right)$ can be computed in either chamber. (4.72) is in perfect agreement with (2.42), showing that the Boltzmann gas picture correctly reproduces the semi-primitive motivic wall-crossing formula. To compare (4.72) with known results, we note that in terms of the "integer" motivic invariants $\Omega_{\text {ref }, n}(\gamma),(4.72)$ becomes an infinite product [34]

$$
Z_{\text {halo }}\left(\gamma_{1}, q, y\right)=\prod_{k=1}^{\infty} \prod_{j=1}^{k\left|\gamma_{12}\right|} \prod_{n \in \mathbb{Z}}\left(1-(-1)^{2 j-k\left|\gamma_{12}\right|} q^{k} y^{n+2 j-1-k\left|\gamma_{12}\right|}\right)^{(-1)^{n} \Omega_{\mathrm{ref}, n}\left(k \gamma_{2}\right)} .
$$


To see this, note that the logarithm of the r.h.s. of (4.73) can be rewritten as

$$
-\sum_{d \geq 1} \sum_{k \geq 1} \sum_{n} \sum_{j=1}^{k\left|\gamma_{12}\right|} \frac{1}{d}(-1)^{n} \Omega_{\mathrm{ref}, n}\left(k \gamma_{2}\right)\left[(-1)^{2 j-k\left|\gamma_{12}\right|} q^{k} y^{n+2 j-1-k\left|\gamma_{12}\right|}\right]^{d} \text {. }
$$

The sum over $n$ leads to $\Omega_{\text {ref, } n}\left(k \gamma_{2}, y^{d}\right)$, while the sum over $j$ is geometric, leading to

$$
\log Z_{\text {halo }}\left(\gamma_{1}, q, y\right)=\sum_{d \geq 1} \sum_{k \geq 1} \frac{1}{d} \Omega_{\text {ref }}\left(k \gamma_{2}, y^{d}\right) \frac{(-y)^{k d \gamma_{12}}-(-y)^{-k d \gamma_{12}}}{y^{d}-y^{-d}} q^{k d}
$$

where we have used $\gamma_{12}<0$ to replace $\left|\gamma_{12}\right|$ by $-\gamma_{12}$. Setting $\ell=k d$, the sum runs over divisors $d$ of $N$ and reproduces (4.72).

The order two and three motivic wall-crossing formula can be obtained by making the replacements (4.69) in eqs.(4.37), (4.51).

Finally, let us consider the problem of determining a generic 3-body contribution to the wall-crossing formula: given three charge vectors $\alpha_{1}, \alpha_{2}$ and $\alpha_{3}$ in $\tilde{\Gamma}$, what is the coefficient of $\bar{\Omega}_{\text {ref }}^{+}\left(\alpha_{1}, y\right) \bar{\Omega}_{\text {ref }}^{+}\left(\alpha_{2}, y\right) \bar{\Omega}_{\text {ref }}^{+}\left(\alpha_{3}, y\right)$ in the expression for $\Delta \bar{\Omega}_{\text {ref }}\left(\alpha_{1}+\alpha_{2}+\alpha_{3}, y\right)$ ? The analysis is a straightforward generalization of that in $\S 4.4$ and the final result is obtained by replacing $\kappa\left(\alpha_{i j}\right)$ by $\kappa\left(\alpha_{i j}, y\right)$ in (4.16). This gives:

$$
\begin{aligned}
& \Delta \bar{\Omega}\left(\alpha_{1}+\alpha_{2}+\alpha_{3}, y\right)=(-1)^{\alpha_{12}+\alpha_{23}+\alpha_{13}}(\sinh \nu)^{-2} \sinh \left(\nu \alpha_{12}\right) \sinh \left(\nu\left(\alpha_{13}+\alpha_{23}\right)\right) \\
& \bar{\Omega}^{+}\left(\alpha_{1}, y\right) \bar{\Omega}^{+}\left(\alpha_{2}, y\right) \bar{\Omega}^{+}\left(\alpha_{3}, y\right)+\cdots .
\end{aligned}
$$

This is in perfect agreement with (3.10) computed from the spectrum of bound states of a 3centered configuration in supergravity. Similarly the 4-body contribution can be computed by replacing $\kappa(\alpha)$ by $\kappa(\alpha, y)$ in (4.18). The result is in perfect agreement with (3.14) and (3.51). We have also carried out a similar computation for 5-body contribution and compared with the results obtained by following the procedure of $\S 3$, but we shall not give the details.

\subsection{KS vs. supergravity}

Eventually one would like to prove that the KS wall crossing formula given in (4.65) is equivalent to the one obtained from quantization of multi-black hole solutions as given in (1.5), (1.6). We have not yet reached this goal, but would like to point out some common aspects of these two formulæ. The summand in (1.6) depends analytically on the $\alpha_{i j}$ 's, but the analyticity of the sum is broken by the third condition described below (1.6). In particular this constraint measures whether $\sum_{a} \beta^{(a)}$, represented as a vector in the two dimensional plane in the convention described below (3.1), lies to the left or the right of the vector $\alpha_{1}+\cdots+\alpha_{n}$. Let us denote by $\mathcal{B}$ the set of all vectors of the form $\sum_{i} m_{i} \alpha_{i}$ with $m_{i}=0$ or 1 , and arrange them in an order following the convention described below (3.1). Let $\mathcal{B}^{\prime}$ denote the subset of elements of $\mathcal{B}$ which lie to the left of the central element $\alpha_{1}+\cdots+\alpha_{n}$. In this case the expression for $g_{\text {ref }}$ given in (1.6) depends on the subset $\mathcal{B}^{\prime}$. As we vary the $\alpha_{i}$ 's this subset may change and in that case $g_{\text {ref }}$ will be given by a 
different analytic function of the $\alpha_{i j}$ 's. Note however that $g_{\text {ref }}$ does not depend on the relative ordering of the vectors inside the subset $\mathcal{B}^{\prime}$.

Now this lack of analyticity is also manifest in the KS wall-crossing formula. To see this, recall the procedure for manipulating the KS formula given in $\S 4.4$. Here we are supposed to begin with the product $e_{\alpha_{n}} \cdots e_{\alpha_{1}}$ and bring it to the order in which the vectors appear in the set $\mathcal{B}$. Changing this order leads to a different final order of the $e_{\beta}$ 's and hence we expect $g_{\mathrm{ref}}\left(\alpha_{1}, \ldots, \alpha_{n}, y\right)$, given by the coefficient of $e_{\alpha_{1}+\cdots+\alpha_{n}}$ in the final state, to change. This in turn prevents $g_{\text {ref }}$ to be given by an analytic formula involving the $\alpha_{i j}$ 's for all choices of $\alpha_{i}$.

Now, the KS prescription for computing $g_{\text {ref }}$ a priori seems to depend on more information than (1.6) since the KS formula requires the detailed ordering of the vectors in $\mathcal{B}$, rather than just the unordered list of the ones which lie to the left of $\alpha_{1}+\cdots+\alpha_{n}$. We shall now show that the KS formula in fact only depends on the unordered list of vectors which lie to the left of $\alpha_{1}+\cdots+\alpha_{n}$. For this let us consider a given order of all the $\alpha_{i}$ 's and supppose that we have brought $e_{\alpha_{n}} \cdots e_{\alpha_{1}}$ to the required order. Now consider the effect of switching the relative order between two neighbouring vectors $\beta_{1}$ and $\beta_{2}$ on the left of $\alpha_{1}+\cdots+\alpha_{n}$. This will require to switch the corresponding $e_{\beta_{1}}$ and $e_{\beta_{2}}$ and will produce an extra factor of $e_{\beta_{1}+\beta_{2}}$. But since $\beta_{1}$ and $\beta_{2}$ both lie to the left of $\alpha_{1}+\cdots+\alpha_{n}$, $\beta_{1}+\beta_{2}$ will also lie to the left of $\alpha_{1}+\cdots+\alpha_{n}$. Thus such switchings can never produce a factor of $e_{\alpha_{1}+\cdots+\alpha_{n}}$. The same argument holds if we switch two vectors on the right of $\alpha_{1}+\cdots+\alpha_{n}$. Thus a term proportional to $e_{\alpha_{1}+\cdots+\alpha_{n}}$ can arise only if we switch a vector from the left of $\alpha_{1}+\ldots \alpha_{n}$ with a vector to the right of $\alpha_{1}+\cdots+\alpha_{n}$. This shows that the non-analyticity of (1.6) and the KS formula are controlled by the same data.

\section{$5 \quad$ Wall-crossing from the Joyce-Song formula}

In their work on Donaldson-Thomas invariants for coherent sheaves on a Calabi-Yau threefold $\mathcal{X}[15]$ (which presumably count D6-D4-D2-D0 bound states in type IIA string theory compactified on $\mathcal{X}$ ), Joyce and Song give a fully explicit expression for the rational DT invariants $\bar{\Omega}^{-}$on one side of the wall, in terms of the rational DT invariants $\bar{\Omega}^{+}$on the other side. Thus, the JS formula can be viewed as the solution to the implicit relation given by KS. In particular, it directly provides the functions $g\left(\left\{\alpha_{i}\right\}\right)$ appearing in (2.16), i.e. the solution to the black hole bound state problem. It should be noted however that the JS wall-crossing formula involves sums over many terms with large denominators and large cancellations, and is less computationally efficient that the KS formula (compare for instance table (5.16) with the analogous computation for KS given in eq. (4.18)). In addition, the simple rule for dealing with identical particles mentioned at the end of $\S 4.4$ is not at all obvious from the JS formula.

One way of understanding the large redundancy is that JS work with Abelian categories, where constituents are either a subobject or a quotient object of the complete object. In physical terms this means that different terms in the JS wall-crossing formula keep track of the order in which the constituents (molecules) make the complete object (bound molecular cluster). But in physics (and in the derived category on which KS anal- 
ysis is based) such a distinction is not present. For example the existence and index of a bound state of two primitive constituents carrying charges $\gamma_{1}$ and $\gamma_{2}$ is independent of the order in which we choose $\gamma_{1}$ and $\gamma_{2}$. For this reason, the JS formula contains many terms which must combine at the end to ensure the independence of the final answer of the order in which the constituents are chosen. The KS formula is less redundant, at the cost of being implicit and perhaps less rigorously established.

After stating the JS formula in $\S 5.1$ and its implication for the black hole bound state problem in $\S 5.2$, we verify the equivalence of the JS and KS formulæ for generic three and four body contribution, and semi-primitive wall-crossing in $\S 5.3$ and $\S 5.4$.

\subsection{Statement of the JS formula}

In $[14,15]$, the authors define rational-valued generalized Donaldson-Thomas invariants $\overline{\mathrm{DT}}^{\gamma}(\tau)$ for any class $\gamma \in C(\mathcal{X})$, where $\mathcal{X}$ is a Calabi-Yau three-fold, $C(\mathcal{X})$ is a positive cone $^{17}$ inside $K(\mathcal{X})$ and $\tau$ is a stability condition. They furthermore establish a general wall-crossing formula for the variation of $\overline{\mathrm{DT}}^{\gamma}(\tau)$ under a change of stability condition. Conjecturally, the rational invariants $\overline{\mathrm{DT}}^{\gamma}(\tau)$ are related to integer invariants $\mathrm{DT}^{\gamma}(\tau)$ by a relation identical to $(1.2)$, and $\overline{\mathrm{DT}}^{\gamma}(\tau), \mathrm{DT}^{\gamma}(\tau)$ are particular instance of the invariants $\bar{\Omega}(\gamma ; t), \Omega(\gamma ; t)$ considered in [11]. The stability condition $\tau$ is determined by a point in Kähler moduli space $t$. In the convention we have chosen,

$$
\tau(\gamma, t)=-\arg Z(\gamma, t)
$$

In this section we shall assume that the conjectured relation between the rational and integer invariants holds, and denote the rational DT invariants of $[14,15]$ as $\bar{\Omega}(\gamma ; t)$. The JS wall-crossing formula then furnishes the solution to the KS formula (4.5), i.e. expresses $\bar{\Omega}^{-}(\gamma)$ in terms of $\bar{\Omega}^{+}\left(\gamma^{\prime}\right)$.

Let $\alpha_{1}, \alpha_{2}, \ldots, \alpha_{n}$ be $n$ charge vectors in the positive cone $C(\mathcal{X})$ inside the charge lattice described by eq, $(2.5)$, and $(t, \tilde{t})$ be a pair of points on the Kähler moduli space with associated stability conditions $(\tau, \tilde{\tau})$. To express the JS wall-crossing formula, we first need to introduce two functions $S\left(\alpha_{1}, \ldots, \alpha_{n} ; t, \tilde{t}\right)$ and $U\left(\alpha_{1}, \ldots, \alpha_{n} ; t, \tilde{t}\right)$, whose role is to capture the relevant information about the ordering of phases of $\left\{Z_{\alpha_{i}}\right\}$. We define $S\left(\alpha_{1}, \ldots, \alpha_{n} ; t, \tilde{t}\right) \in\{0, \pm 1\}$ as follows. If $n=1$, set $S\left(\alpha_{1} ; t, \tilde{t}\right)=1$. If $n>1$ and, for every $i=1, \ldots, n-1$, either

(a) $\quad \tau\left(\alpha_{i}\right) \leq \tau\left(\alpha_{i+1}\right)$ and $\quad \tilde{\tau}\left(\alpha_{1}+\cdots+\alpha_{i}\right)>\tilde{\tau}\left(\alpha_{i+1}+\cdots+\alpha_{n}\right), \quad$ or

(b) $\quad \tau\left(\alpha_{i}\right)>\tau\left(\alpha_{i+1}\right)$ and $\tilde{\tau}\left(\alpha_{1}+\cdots+\alpha_{i}\right) \leq \tilde{\tau}\left(\alpha_{i+1}+\cdots+\alpha_{n}\right)$,

let $S\left(\alpha_{1}, \ldots, \alpha_{n} ; t, \tilde{t}\right)=(-1)^{r}$, where $r$ is the number of times option (a) is realized; otherwise, $S\left(\alpha_{1}, \ldots, \alpha_{n} ; t, \tilde{t}\right)=0$.

To define $U\left(\alpha_{1}, \ldots, \alpha_{n} ; t, \tilde{t}\right)$, consider all ordered partitions of the $n$ vectors $\alpha_{i}$ into $1 \leq m \leq n$ packets $\left\{\alpha_{a_{j-1}+1}, \cdots, \alpha_{a_{j}}\right\}, j=1, \ldots, m$, with $0=a_{0}<a_{1}<\cdots<a_{m}=n$, such that all vectors in each packet have the same phase $\tau\left(\alpha_{i}\right)$. Let $\beta_{j}=\alpha_{a_{j-1}+1}+\cdots+$

\footnotetext{
${ }^{17}$ This is the analog of the wedge $\tilde{\Gamma}$ introduced in eq. (2.5).
} 
$\alpha_{a_{j}}, j=1, \ldots, m$ be the sum of the charge vectors in each packet. We refer to the ordered set $\left\{\beta_{j}, j=1, \ldots, m\right\}$ as a contraction of $\left\{\alpha_{i}\right\}$. Next, consider all ordered partitions of the $m$ vectors $\beta_{j}$ into $1 \leq l \leq m$ packets $\left\{\beta_{b_{k-1}+1}, \cdots, \beta_{b_{k}}\right\}$, with $0=b_{0}<b_{1}<\cdots<b_{l}=m$, $k=1, \ldots, l$, such that the total charge vectors $\delta_{k}=\beta_{b_{k-1}+1}+\cdots+\beta_{b_{k}}, k=1, \ldots, l$ in each packets all have the same phase $\tilde{\tau}\left(\delta_{k}\right)$ (which is then equal to $\tilde{\tau}\left(\sum \alpha_{i}\right)$ ). Now associate to each of the $l$ packets in the contraction a factor $S\left(\beta_{b_{k-1}+1}, \beta_{b_{k-1}+2}, \ldots, \beta_{b_{k}} ; t, \tilde{t}\right)$ as defined above, and define the $U$-factor as the sum

$$
U\left(\alpha_{1}, \ldots, \alpha_{n} ; t, \tilde{t}\right) \equiv \sum \frac{(-1)^{l-1}}{l} \cdot \prod_{k=1}^{l} S\left(\beta_{b_{k-1}+1}, \beta_{b_{k-1}+2}, \ldots, \beta_{b_{k}} ; t, \tilde{t}\right) \cdot \prod_{j=1}^{m} \frac{1}{\left(a_{j}-a_{j-1}\right) !}
$$

over all partitions of $\alpha_{i}$ and partitions of $\beta_{j}$ satisfying the conditions above.

Finally, departing slightly from the presentation in [14], let us define the $\mathcal{L}$ factor

$$
\mathcal{L}\left(\alpha_{1}, \ldots, \alpha_{n}\right) \equiv \sum_{\substack{\text { connected oriented trees: } \\ \text { vertices }\{1, \ldots, n\}, \text { edge } i \rightarrow j, \text { implies } i<j}} \prod_{\text {edges } i \rightarrow j}\left\langle\alpha_{i}, \alpha_{j}\right\rangle,
$$

where the sum runs over all connected trees $g$ with $n$ vertices labelled from $i=1$ to $i=n$. We denote by $g^{(0)}=\{1, \ldots, n\}$ the set of vertices, and by $g^{(1)}$ the set of oriented edges $(i, j)$, with the orientation inherited from the standard order $i<j$ on $g^{(0)}$. In other words given any labelled tree, and an edge of this tree connecting $i$ to $j$, we associate to this edge an orientation from $i \rightarrow j$ if $i<j$. In order to implement this formula on a computer, it is useful to note that there are $n^{(n-2)}$ labeled trees with $n$ vertices, which are in one-to-one correspondence with their Prüfer code, an element in $\{1, \ldots, n\}^{n-2} .^{18}$

Having defined the $S, U$ and $\mathcal{L}$ factors, we can now state the JS wall-crossing formula (eq. (5.13) in [14]):

$$
\bar{\Omega}(\gamma ; \tilde{t})=\sum_{n \geq 1} \sum_{\substack{\left(\alpha_{1}, \ldots, \alpha_{n}\right) \in C(\mathcal{X}) \\ \gamma=\alpha_{1}+\cdots+\alpha_{n}}} \frac{(-1)^{n-1+\sum_{i<j}\left|\left\langle\alpha_{i}, \alpha_{j}\right\rangle\right|}}{2^{n-1}} U\left(\alpha_{1}, \ldots, \alpha_{n} ; t, \tilde{t}\right) \mathcal{L}\left(\alpha_{1}, \ldots, \alpha_{n}\right) \prod_{i=1}^{n} \bar{\Omega}\left(\alpha_{i}, t\right),
$$

where the second sum runs over all ordered decompositions $\gamma=\alpha_{1}+\cdots+\alpha_{n}$ with $\alpha_{i} \in$ $C(\mathcal{X})$. Note that due to this constraint, eq, (5.5) is automatically consistent with the charge conservation property.

The JS formula is valid for any pair of points in the moduli space with stability conditions $\tau, \tilde{\tau}$. Now we restrict to the vicinity of a wall of marginal stability $\mathcal{P}\left(\gamma_{1}, \gamma_{2}\right)$. The only states whose BPS invariants can jump are those whose charges lie in $\tilde{\Gamma} \subset C(\mathcal{X})$. Moreover, their discontinuities only depend on the BPS invariants of states in $\tilde{\Gamma}$. We denote the states $\gamma=M \gamma_{1}+N \gamma_{2}$ by $\gamma=(M, N)$. As in previous section, let us assume that $\gamma_{12}<0$, and take $t$ in the chamber $c^{+}$where multi-centered configurations are absent,

\footnotetext{
${ }^{18}$ Note that these labeled trees differ qualitatively from the attractor flow trees. One can view these labeled trees as connecting the endpoints of the attractor flow trees.
} 

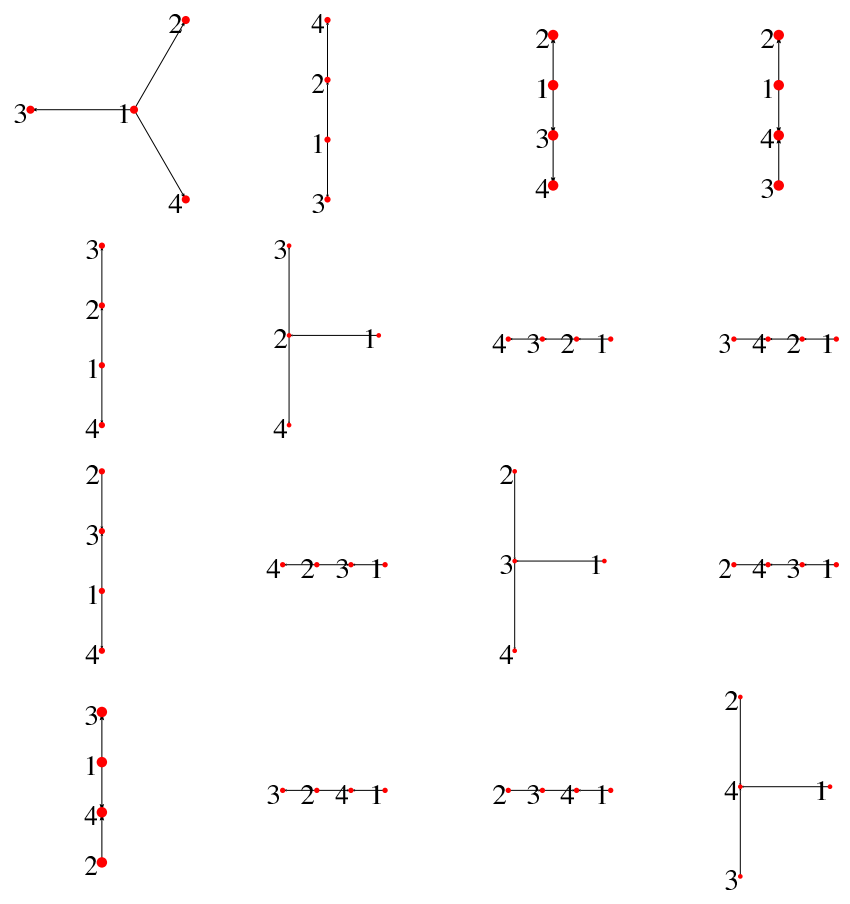

Figure 1. The 16 labelled trees contributing to $\mathcal{L}\left(\alpha_{1}, \alpha_{2}, \alpha_{3}, \alpha_{4}\right)$.

and $\tilde{t}$ in the chamber $c^{-}$where they are present. From (2.6), we see that, in the vicinity of the wall,

$$
\pm\left\langle\gamma, \gamma^{\prime}\right\rangle\left[\tau\left(\gamma, c_{ \pm}\right)-\tau\left(\gamma^{\prime} ; c_{ \pm}\right)\right]>0
$$

Therefore, the conditions (5.2) become

(a) $\left\langle\alpha_{i}, \alpha_{i+1}\right\rangle \leq 0$ and $\left\langle\alpha_{1}+\cdots+\alpha_{i}, \alpha_{i+1}+\cdots+\alpha_{n}\right\rangle<0, \quad$ or

(b) $\left\langle\alpha_{i}, \alpha_{i+1}\right\rangle>0$ and $\left\langle\alpha_{1}+\cdots+\alpha_{i}, \alpha_{i+1}+\cdots+\alpha_{n}\right\rangle \geq 0$,

and (5.5) gives an expression for $\bar{\Omega}^{-}$in terms of $\bar{\Omega}^{+}$.

To illustrate the afore going prescription, we evaluate in detail the term proportional to $\bar{\Omega}^{+}\left(\gamma_{1}\right)^{2} \bar{\Omega}^{+}\left(\gamma_{2}\right)^{2}$ in the expressions for $\bar{\Omega}^{-}\left(2 \gamma_{1}+2 \gamma_{2}\right)$. We first evaluate the $U$ factors,

$$
\begin{aligned}
& U\left(\gamma_{2}, \gamma_{2}, \gamma_{1}, \gamma_{1}\right)=\frac{1}{4} S\left(2 \gamma_{2}, 2 \gamma_{1}\right)+\frac{1}{2} S\left(\gamma_{2}, \gamma_{2}, 2 \gamma_{1}\right)+\frac{1}{2} S\left(2 \gamma_{2}, \gamma_{1}, \gamma_{1}\right)+S\left(\gamma_{2}, \gamma_{2}, \gamma_{1}, \gamma_{1}\right)=\frac{1}{4} \\
& U\left(\gamma_{2}, \gamma_{1}, \gamma_{2}, \gamma_{1}\right)=S\left(\gamma_{2}, \gamma_{1}, \gamma_{2}, \gamma_{1}\right)-\frac{1}{2} S\left(\gamma_{2}, \gamma_{1}\right)^{2}=-\frac{1}{2} \\
& U\left(\gamma_{2}, \gamma_{1}, \gamma_{1}, \gamma_{2}\right)=-\frac{1}{2} S\left(\gamma_{1}, \gamma_{2}\right) S\left(\gamma_{2}, \gamma_{1}\right)+\frac{1}{2} S\left(\gamma_{2}, 2 \gamma_{1}, \gamma_{2}\right)+S\left(\gamma_{2}, \gamma_{1}, \gamma_{1}, \gamma_{2}\right)=0 \\
& U\left(\gamma_{1}, \gamma_{2}, \gamma_{2}, \gamma_{1}\right)=-\frac{1}{2} S\left(\gamma_{1}, \gamma_{2}\right) S\left(\gamma_{2}, \gamma_{1}\right)+\frac{1}{2} S\left(\gamma_{1}, 2 \gamma_{2}, \gamma_{1}\right)+S\left(\gamma_{1}, \gamma_{2}, \gamma_{2}, \gamma_{1}\right)=0 \\
& U\left(\gamma_{1}, \gamma_{2}, \gamma_{1}, \gamma_{2}\right)=S\left(\gamma_{1}, \gamma_{2}, \gamma_{1}, \gamma_{2}\right)-\frac{1}{2} S\left(\gamma_{1}, \gamma_{2}\right)^{2}=\frac{1}{2} \\
& U\left(\gamma_{1}, \gamma_{1}, \gamma_{2}, \gamma_{2}\right)=\frac{1}{4} S\left(2 \gamma_{1}, 2 \gamma_{2}\right)+\frac{1}{2} S\left(\gamma_{1}, \gamma_{1}, 2 \gamma_{2}\right)+\frac{1}{2} S\left(2 \gamma_{1}, \gamma_{2}, \gamma_{2}\right)+S\left(\gamma_{1}, \gamma_{1}, \gamma_{2}, \gamma_{2}\right)=-\frac{1}{4}
\end{aligned}
$$


For example in the first line the only non-vanishing contribution comes from the $S\left(2 \gamma_{2}, 2 \gamma_{1}\right) / 4$ term. For the other terms the condition (5.7) fails for at least one $i$. To compute the $\mathcal{L}$ factors we observe that there are 16 trees with 4 labelled nodes (see figure 1). Out of those, twelve are obtained by various inequivalent permutations of the tree connecting nodes 1 to 2, 2 to 3 and 3 to 4 , and four are obtained by inequivalent permutations of a tree that connects node 1 to each of the nodes 2,3 and 4 . The $\mathcal{L}$-factors are computed by adding the contributions from each of these 16 trees, leading to

$$
\begin{aligned}
\mathcal{L}\left(\alpha_{1}, \alpha_{2}, \alpha_{3}, \alpha_{4}\right)= & \alpha_{12} \alpha_{13} \alpha_{14}+\alpha_{12} \alpha_{23} \alpha_{14}+\alpha_{13} \alpha_{23} \alpha_{14}+\alpha_{13} \alpha_{24} \alpha_{14} \\
& +\alpha_{23} \alpha_{24} \alpha_{14}+\alpha_{12} \alpha_{34} \alpha_{14}+\alpha_{23} \alpha_{34} \alpha_{14}+\alpha_{24} \alpha_{34} \alpha_{14} \\
& +\alpha_{12} \alpha_{13} \alpha_{24}+\alpha_{12} \alpha_{23} \alpha_{24}+\alpha_{13} \alpha_{23} \alpha_{24}+\alpha_{12} \alpha_{13} \alpha_{34} \\
& +\alpha_{12} \alpha_{23} \alpha_{34}+\alpha_{13} \alpha_{23} \alpha_{34}+\alpha_{12} \alpha_{24} \alpha_{34}+\alpha_{13} \alpha_{24} \alpha_{34} .
\end{aligned}
$$

This gives

$$
\begin{aligned}
& \mathcal{L}\left(\gamma_{2}, \gamma_{2}, \gamma_{1}, \gamma_{1}\right)=-4 \gamma_{12}^{3}, \quad \mathcal{L}\left(\gamma_{2}, \gamma_{1}, \gamma_{2}, \gamma_{1}\right)=2 \gamma_{12}^{3} \\
& \mathcal{L}\left(\gamma_{2}, \gamma_{1}, \gamma_{1}, \gamma_{2}\right)=0, \quad \mathcal{L}\left(\gamma_{1}, \gamma_{2}, \gamma_{2}, \gamma_{1}\right)=0 \\
& \mathcal{L}\left(\gamma_{1}, \gamma_{2}, \gamma_{1}, \gamma_{2}\right)=-2 \gamma_{12}^{3}, \quad \mathcal{L}\left(\gamma_{1}, \gamma_{1}, \gamma_{2}, \gamma_{2}\right)=4 \gamma_{12}^{3}
\end{aligned}
$$

The total contribution from these terms to the right hand side of (5.5) is thus given by

$$
\bar{\Omega}^{-}\left(2 \gamma_{1}+2 \gamma_{2}\right)=\frac{1}{2} \gamma_{12}^{3}\left[\bar{\Omega}^{+}\left(\gamma_{1}\right)\right]^{2}\left[\bar{\Omega}^{+}\left(\gamma_{2}\right)\right]^{2}+\ldots,
$$

in agreement with the formulæ (4.37) and (A.2).

\subsection{Index of supersymmetric bound states from the JS formula}

It is useful to rewrite the JS formula (5.5) as a sum over unordered decompositions $\left\{\alpha_{i}\right\}$ of the charge vector $\gamma$,

$$
\Delta \bar{\Omega}(\gamma)=\sum_{n \geq 2} \sum_{\substack{\left\{\alpha_{1}, \ldots, \alpha_{n}\right\} \in C(\mathcal{X}) \\ \gamma=\alpha_{1}+\cdots+\alpha_{n}}} \frac{g\left(\left\{\alpha_{i}\right\}\right)}{\left|\operatorname{Aut}\left(\left\{\alpha_{i}\right\}\right)\right|} \prod_{i=1}^{n} \bar{\Omega}^{+}\left(\alpha_{i}\right) .
$$

where $\left|\operatorname{Aut}\left(\left\{\alpha_{i}\right\}\right)\right|$ is defined as follows. We choose any specific order of the $\alpha_{i}$ 's and identify $\left|\operatorname{Aut}\left(\left\{\alpha_{i}\right\}\right)\right|$ as the order of the subgroup of the permutation group $\Sigma_{n}$ on $n$ elements which leaves invariant this particular ordering of the $\left\{\alpha_{i}\right\}$ 's. In particular if all the $\alpha_{i}$ 's are different then the automorphism subgroup is trivial and $\left|\operatorname{Aut}\left(\left\{\alpha_{i}\right\}\right)\right|=1$. On the other hand if $m$ of the $\alpha_{i}$ 's are the same then $\left|\operatorname{Aut}\left(\left\{\alpha_{i}\right\}\right)\right|=m$ !. The coefficient $g\left(\left\{\alpha_{i}\right\}\right)$ is then given by

$$
g\left(\left\{\alpha_{i}\right\}\right)=\frac{(-1)^{n-1+\sum_{i<j}\left\langle\alpha_{i}, \alpha_{j}\right\rangle}}{2^{n-1}} \sum_{\sigma \in \Sigma_{n}} U\left(\alpha_{\sigma(1)}, \ldots, \alpha_{\sigma(n)} ; t_{+}, t_{-}\right) \mathcal{L}\left(\alpha_{\sigma(1)}, \ldots, \alpha_{\sigma(n)}\right),
$$

where the sum runs over all permutations of $n$ elements. Relabelling the set $\left\{\alpha_{i}\right\}$ into a partition $\left\{m_{r, s}\right\}$ such that $\gamma=(M, N)=\sum_{r, s}(r, s) m_{r, s}$, the coefficient $g\left(\left\{\alpha_{i}\right\}\right)$ is the same as the one appearing in (2.16). Thus it should be identified with the index of the supersymmetric quantum mechanics of $n$ distinguishable charged dyons in $\mathbb{R}^{1,3}$, along the lines of $[8]$. 


\subsection{Generic 2-body, 3-body and 4-body contributions}

Let us first derive the primitive wall-crossing formula $\gamma \rightarrow \gamma_{1}+\gamma_{2}$ from the JS wall crossing formula. In this case there is only one tree - connecting the nodes 1 and 2 with the arrow directed from 1 to 2. If we choose the first node to be $\gamma_{1}$ and the second node to be $\gamma_{2}$ then since $\gamma_{12}<0$ possibility (a) in (5.7) is realized, and we have $U\left(\gamma_{1}, \gamma_{2}\right)=S\left(\gamma_{1}, \gamma_{2}\right)=$ -1 . Since $n=2$, (5.13) now gives a contribution of $\frac{1}{2}(-1)^{1+\gamma_{12}} \gamma_{12}(-1)$. An identical contribution comes from the term where we put $\gamma_{2}$ in the first node and $\gamma_{1}$ in the second node since now we have $U=S=1$ and the $\left\langle\alpha_{\sigma(i)}, \alpha_{\sigma(j)}\right\rangle$ factor in (5.13) is now equal to $\gamma_{21}$. Adding the two contributions we recover the primitive wall-crossing formula (4.15). We summarize the $S, U$ and $\mathcal{L}$ factors for the two permutations in the table below.

\begin{tabular}{|c|c|c|c|}
\hline$\sigma(12)$ & $S$ & $U$ & $\mathcal{L}$ \\
\hline 12 & $a$ & -1 & $\gamma_{12}$ \\
21 & $b$ & 1 & $-\gamma_{12}$ \\
\hline
\end{tabular}

Next we reproduce the result of section 4.4 for the generic three body contribution to the wall-crossing from centers carrying charges $\left(\alpha_{1}, \alpha_{2}, \alpha_{3}\right)$. The order of the $\alpha_{i}$ 's is given by (3.8). With the definition of the ordering explained below (3.1) we see that if $(\alpha, \beta)$ follow a clockwise order then $\langle\alpha, \beta\rangle>0$. Since the phases are assumed to be generic, the $U$ and $S$ factors coincide, i.e. $U\left(\alpha_{i}, \alpha_{j}, \alpha_{k} ; t, \tilde{t}\right)=S\left(\alpha_{i}, \alpha_{j}, \alpha_{k} ; t, \tilde{t}\right)$, for any permutation $\{i, j, k\}$ of $\{1,2,3\}$. In this case, there are three trees contributing. The $S, U, \mathcal{L}$-factors are summarized in the table below. Substitution of these data into eq. (5.5) reproduces directly eqs. (3.11), (4.17).

\begin{tabular}{|c|c|c|c|}
\hline$\sigma(123)$ & $S$ & $U$ & $\mathcal{L}$ \\
\hline 123 & $\mathrm{bb}$ & 1 & $\alpha_{12} \alpha_{13}+\alpha_{13} \alpha_{23}+\alpha_{12} \alpha_{23}$ \\
132 & $\mathrm{~b}-$ & 0 & $\alpha_{12} \alpha_{13}-\alpha_{13} \alpha_{23}-\alpha_{12} \alpha_{23}$ \\
213 & $\mathrm{ab}$ & -1 & $-\alpha_{12} \alpha_{23}+\alpha_{13} \alpha_{23}-\alpha_{12} \alpha_{13}$ \\
231 & $-\mathrm{a}$ & 0 & $\alpha_{12} \alpha_{13}-\alpha_{13} \alpha_{23}-\alpha_{12} \alpha_{23}$ \\
312 & $\mathrm{ab}$ & -1 & $\alpha_{13} \alpha_{23}-\alpha_{12} \alpha_{23}-\alpha_{13} \alpha_{12}$ \\
321 & $\mathrm{aa}$ & 1 & $\alpha_{13} \alpha_{23}+\alpha_{12} \alpha_{13}+\alpha_{12} \alpha_{23}$ \\
\hline
\end{tabular}

Next, we turn to the generic 4-body case. We use the same phase ordering as eq. (3.13). 
The non-vanishing contributions are given in the following table

\begin{tabular}{|c|c|c|c|}
\hline$\sigma(1234)$ & $S$ & $U$ & $\mathcal{L}$ \\
\hline 1234 & $\mathrm{bbb}$ & 1 & $\begin{array}{l}\alpha_{12} \alpha_{13} \alpha_{14}+\alpha_{12} \alpha_{23} \alpha_{14}+\alpha_{13} \alpha_{23} \alpha_{14}+\alpha_{13} \alpha_{24} \alpha_{14} \\
+\alpha_{23} \alpha_{24} \alpha_{14}+\alpha_{12} \alpha_{34} \alpha_{14}+\alpha_{23} \alpha_{34} \alpha_{14}+\alpha_{24} \alpha_{34} \alpha_{14} \\
+\alpha_{12} \alpha_{13} \alpha_{24}+\alpha_{12} \alpha_{23} \alpha_{24}+\alpha_{13} \alpha_{23} \alpha_{24}+\alpha_{12} \alpha_{13} \alpha_{34} \\
+\alpha_{12} \alpha_{23} \alpha_{34}+\alpha_{13} \alpha_{23} \alpha_{34}+\alpha_{12} \alpha_{24} \alpha_{34}+\alpha_{13} \alpha_{24} \alpha_{34}\end{array}$ \\
\hline 1342 & $\mathrm{bba}$ & -1 & $\begin{array}{l}\alpha_{12} \alpha_{13} \alpha_{14}-\alpha_{12} \alpha_{23} \alpha_{14}-\alpha_{13} \alpha_{23} \alpha_{14}-\alpha_{13} \alpha_{24} \alpha_{14} \\
+\alpha_{23} \alpha_{24} \alpha_{14}+\alpha_{12} \alpha_{34} \alpha_{14}-\alpha_{23} \alpha_{34} \alpha_{14}-\alpha_{24} \alpha_{34} \alpha_{14} \\
-\alpha_{12} \alpha_{13} \alpha_{24}+\alpha_{12} \alpha_{23} \alpha_{24}+\alpha_{13} \alpha_{23} \alpha_{24}+\alpha_{12} \alpha_{13} \alpha_{34} \\
-\alpha_{12} \alpha_{23} \alpha_{34}-\alpha_{13} \alpha_{23} \alpha_{34}-\alpha_{12} \alpha_{24} \alpha_{34}-\alpha_{13} \alpha_{24} \alpha_{34}\end{array}$ \\
\hline 1423 & bab & -1 & $\begin{array}{l}\alpha_{12} \alpha_{13} \alpha_{14}+\alpha_{12} \alpha_{23} \alpha_{14}+\alpha_{13} \alpha_{23} \alpha_{14}-\alpha_{13} \alpha_{24} \alpha_{14} \\
-\alpha_{23} \alpha_{24} \alpha_{14}-\alpha_{12} \alpha_{34} \alpha_{14}-\alpha_{23} \alpha_{34} \alpha_{14}+\alpha_{24} \alpha_{34} \alpha_{14} \\
-\alpha_{12} \alpha_{13} \alpha_{24}-\alpha_{12} \alpha_{23} \alpha_{24}-\alpha_{13} \alpha_{23} \alpha_{24}-\alpha_{12} \alpha_{13} \alpha_{34} \\
-\alpha_{12} \alpha_{23} \alpha_{34}-\alpha_{13} \alpha_{23} \alpha_{34}+\alpha_{12} \alpha_{24} \alpha_{34}+\alpha_{13} \alpha_{24} \alpha_{34}\end{array}$ \\
\hline 1432 & baa & 1 & $\begin{array}{l}\alpha_{12} \alpha_{13} \alpha_{14}-\alpha_{12} \alpha_{23} \alpha_{14}-\alpha_{13} \alpha_{23} \alpha_{14}-\alpha_{13} \alpha_{24} \alpha_{14} \\
+\alpha_{23} \alpha_{24} \alpha_{14}-\alpha_{12} \alpha_{34} \alpha_{14}+\alpha_{23} \alpha_{34} \alpha_{14}+\alpha_{24} \alpha_{34} \alpha_{14} \\
-\alpha_{12} \alpha_{13} \alpha_{24}+\alpha_{12} \alpha_{23} \alpha_{24}+\alpha_{13} \alpha_{23} \alpha_{24}-\alpha_{12} \alpha_{13} \alpha_{34} \\
+\alpha_{12} \alpha_{23} \alpha_{34}+\alpha_{13} \alpha_{23} \alpha_{34}+\alpha_{12} \alpha_{24} \alpha_{34}+\alpha_{13} \alpha_{24} \alpha_{34}\end{array}$ \\
\hline 2341 & bba & -1 & {$\left[\begin{array}{l}-\alpha_{12} \alpha_{13} \alpha_{14}+\alpha_{12} \alpha_{23} \alpha_{14}+\alpha_{13} \alpha_{23} \alpha_{14}+\alpha_{13} \alpha_{24} \alpha_{14} \\
-\alpha_{23} \alpha_{24} \alpha_{14}+\alpha_{12} \alpha_{34} \alpha_{14}-\alpha_{23} \alpha_{34} \alpha_{14}-\alpha_{24} \alpha_{34} \alpha_{14} \\
+\alpha_{12} \alpha_{13} \alpha_{24}-\alpha_{12} \alpha_{23} \alpha_{24}-\alpha_{13} \alpha_{23} \alpha_{24}+\alpha_{12} \alpha_{13} \alpha_{34} \\
-\alpha_{12} \alpha_{23} \alpha_{34}-\alpha_{13} \alpha_{23} \alpha_{34}-\alpha_{12} \alpha_{24} \alpha_{34}-\alpha_{13} \alpha_{24} \alpha_{34}\end{array}\right.$} \\
\hline 2413 & bab & -1 & $\begin{array}{l}\alpha_{12} \alpha_{13} \alpha_{14}+\alpha_{12} \alpha_{23} \alpha_{14}-\alpha_{13} \alpha_{23} \alpha_{14}-\alpha_{13} \alpha_{24} \alpha_{14} \\
-\alpha_{23} \alpha_{24} \alpha_{14}-\alpha_{12} \alpha_{34} \alpha_{14}+\alpha_{23} \alpha_{34} \alpha_{14}+\alpha_{24} \alpha_{34} \alpha_{14} \\
-\alpha_{12} \alpha_{13} \alpha_{24}-\alpha_{12} \alpha_{23} \alpha_{24}+\alpha_{13} \alpha_{23} \alpha_{24}+\alpha_{12} \alpha_{13} \alpha_{34} \\
+\alpha_{12} \alpha_{23} \alpha_{34}-\alpha_{13} \alpha_{23} \alpha_{34}+\alpha_{12} \alpha_{24} \alpha_{34}-\alpha_{13} \alpha_{24} \alpha_{34}\end{array}$ \\
\hline 2431 & baa & 1 & {$\left[\begin{array}{l}-\alpha_{12} \alpha_{13} \alpha_{14}+\alpha_{12} \alpha_{23} \alpha_{14}+\alpha_{13} \alpha_{23} \alpha_{14}+\alpha_{13} \alpha_{24} \alpha_{14} \\
-\alpha_{23} \alpha_{24} \alpha_{14}-\alpha_{12} \alpha_{34} \alpha_{14}+\alpha_{23} \alpha_{34} \alpha_{14}+\alpha_{24} \alpha_{34} \alpha_{14} \\
+\alpha_{12} \alpha_{13} \alpha_{24}-\alpha_{12} \alpha_{23} \alpha_{24}-\alpha_{13} \alpha_{23} \alpha_{24}-\alpha_{12} \alpha_{13} \alpha_{34} \\
+\alpha_{12} \alpha_{23} \alpha_{34}+\alpha_{13} \alpha_{23} \alpha_{34}+\alpha_{12} \alpha_{24} \alpha_{34}+\alpha_{13} \alpha_{24} \alpha_{34}\end{array}\right.$} \\
\hline 3124 & $a b b$ & -1 & {$\left[\begin{array}{l}-\alpha_{12} \alpha_{13} \alpha_{14}-\alpha_{12} \alpha_{23} \alpha_{14}+\alpha_{13} \alpha_{23} \alpha_{14}-\alpha_{13} \alpha_{24} \alpha_{14} \\
-\alpha_{23} \alpha_{24} \alpha_{14}+\alpha_{12} \alpha_{34} \alpha_{14}-\alpha_{23} \alpha_{34} \alpha_{14}+\alpha_{24} \alpha_{34} \alpha_{14} \\
-\alpha_{12} \alpha_{13} \alpha_{24}-\alpha_{12} \alpha_{23} \alpha_{24}+\alpha_{13} \alpha_{23} \alpha_{24}-\alpha_{12} \alpha_{13} \alpha_{34} \\
-\alpha_{12} \alpha_{23} \alpha_{34}+\alpha_{13} \alpha_{23} \alpha_{34}+\alpha_{12} \alpha_{24} \alpha_{34}-\alpha_{13} \alpha_{24} \alpha_{34}\end{array}\right.$} \\
\hline 3142 & aba & 1 & {$\left[\begin{array}{l}-\alpha_{12} \alpha_{13} \alpha_{14}-\alpha_{12} \alpha_{23} \alpha_{14}+\alpha_{13} \alpha_{23} \alpha_{14}+\alpha_{13} \alpha_{24} \alpha_{14} \\
+\alpha_{23} \alpha_{24} \alpha_{14}+\alpha_{12} \alpha_{34} \alpha_{14}-\alpha_{23} \alpha_{34} \alpha_{14}-\alpha_{24} \alpha_{34} \alpha_{14} \\
+\alpha_{12} \alpha_{13} \alpha_{24}+\alpha_{12} \alpha_{23} \alpha_{24}-\alpha_{13} \alpha_{23} \alpha_{24}-\alpha_{12} \alpha_{13} \alpha_{34} \\
-\alpha_{12} \alpha_{23} \alpha_{34}+\alpha_{13} \alpha_{23} \alpha_{34}-\alpha_{12} \alpha_{24} \alpha_{34}+\alpha_{13} \alpha_{24} \alpha_{34}\end{array}\right.$} \\
\hline 3241 & aba & 1 & $\begin{array}{l}-\alpha_{12} \alpha_{13} \alpha_{14}-\alpha_{12} \alpha_{23} \alpha_{14}-\alpha_{13} \alpha_{23} \alpha_{14}+\alpha_{13} \alpha_{24} \alpha_{14} \\
+\alpha_{23} \alpha_{24} \alpha_{14}+\alpha_{12} \alpha_{34} \alpha_{14}+\alpha_{23} \alpha_{34} \alpha_{14}-\alpha_{24} \alpha_{34} \alpha_{14} \\
+\alpha_{12} \alpha_{13} \alpha_{24}+\alpha_{12} \alpha_{23} \alpha_{24}+\alpha_{13} \alpha_{23} \alpha_{24}+\alpha_{12} \alpha_{13} \alpha_{34} \\
+\alpha_{12} \alpha_{23} \alpha_{34}+\alpha_{13} \alpha_{23} \alpha_{34}-\alpha_{12} \alpha_{24} \alpha_{34}-\alpha_{13} \alpha_{24} \alpha_{34}\end{array}$ \\
\hline
\end{tabular}




\begin{tabular}{|c|c|c|c|}
\hline$\sigma(1234)$ & $S$ & $U$ & $\mathcal{L}$ \\
\hline 4213 & aab & 1 & $\alpha_{12} \alpha_{13} \alpha_{14}+\alpha_{12} \alpha_{23} \alpha_{14}-\alpha_{13} \alpha_{23} \alpha_{14}+\alpha_{13} \alpha_{24} \alpha_{14}$ \\
& & & $\alpha_{23} \alpha_{24} \alpha_{14}-\alpha_{12} \alpha_{34} \alpha_{14}+\alpha_{23} \alpha_{34} \alpha_{14}-\alpha_{24} \alpha_{34} \alpha_{14}$ \\
& $+\alpha_{12} \alpha_{13} \alpha_{24}+\alpha_{12} \alpha_{23} \alpha_{24}-\alpha_{13} \alpha_{23} \alpha_{24}+\alpha_{12} \alpha_{13} \alpha_{34}$ \\
& $+\alpha_{12} \alpha_{23} \alpha_{34}-\alpha_{13} \alpha_{23} \alpha_{34}-\alpha_{12} \alpha_{24} \alpha_{34}+\alpha_{13} \alpha_{24} \alpha_{34}$ \\
& & & \\
4321 & aaa $\alpha_{13} \alpha_{14}-\alpha_{12} \alpha_{23} \alpha_{14}-\alpha_{13} \alpha_{23} \alpha_{14}-\alpha_{13} \alpha_{24} \alpha_{14}$ \\
$-\alpha_{23} \alpha_{24} \alpha_{14}-\alpha_{12} \alpha_{34} \alpha_{14}-\alpha_{23} \alpha_{34} \alpha_{14}-\alpha_{24} \alpha_{34} \alpha_{14}$ \\
$-\alpha_{12} \alpha_{13} \alpha_{24}-\alpha_{12} \alpha_{23} \alpha_{24}-\alpha_{13} \alpha_{23} \alpha_{24}-\alpha_{12} \alpha_{13} \alpha_{34}$ \\
$-\alpha_{12} \alpha_{23} \alpha_{34}-\alpha_{13} \alpha_{23} \alpha_{34}-\alpha_{12} \alpha_{24} \alpha_{34}-\alpha_{13} \alpha_{24} \alpha_{34}$ \\
\hline
\end{tabular}

Adding up all these contributions, one finds again the result of eqs. (3.15), (4.18).

\subsection{Semi-primitive wall-crossing formula from JS}

Let us now derive the semi-primitive wall-crossing formula from (5.5), i.e. compute $\bar{\Omega}\left(\gamma ; c_{-}\right)$ in terms of $\bar{\Omega}\left(\gamma ; c_{+}\right)$for $\gamma=(1, N) \in \tilde{\Gamma}$. We mostly follow the discussion in [42], section 3, suitably generalized.

At given order $n$, the most general ordered decomposition $\gamma=\sum_{i=1, \ldots, n} \alpha_{i}$ is

$$
\gamma=\left(0, N_{1}\right)+\cdots+\left(0, N_{i_{*}-1}\right)+\left(1, N_{i_{*}}\right)+\left(0, N_{i_{*}+1}\right)+\cdots+\left(0, N_{n}\right)
$$

where $\left\{N_{i}\right\}$ is a partition of $N$ of length $n, \sum_{1 \leq i \leq n} N_{i}=N$, and $1 \leq i_{*} \leq N$ labels the position of the charge $(1,0)$ in this decomposition. For such a pair $\left(\left\{N_{i}\right\}, i_{*}\right)$, one may check that the $S$ factor is given by

$$
S\left(\left\{N_{i}\right\}, i_{*} ; c_{+}, c_{-}\right)=(-1)^{n-i_{*}}
$$

if $i_{*}=1$ or $i_{*}=2$, and vanishes otherwise. To compute the $U$ factor, note that due to the phase constraints, the ordered partition of $\left\{\alpha_{i}\right\}$ labelled by $\left\{a_{j}\right\}$ must decompose into ordered partitions of the 'head' set $\left\{N_{i}\right\}, i=1, \ldots, i_{*}-1$ and 'tail' set $\left\{N_{i}\right\}, i=i_{*}+1, \ldots, n$, while the vector $\left(1, N_{i_{*}}\right)$ must lie in its own packet. Moreover, since the phases of all the vectors $\beta_{j}$, except the vector $\left(1, N_{i_{*}}\right)$, are the same in the chamber $c_{-}$, all the $\beta_{j}$ 's must be be grouped into a single packet at the second step in order to satisfy the phase constraint on the $\delta_{k}$ 's. Otherwise the packet containing $\left(1, N_{i_{*}}\right)$ will have different phase from the others. The $U$-factor therefore reduces to

$$
U\left(\left\{N_{i}\right\}, i_{*} ; c_{+}, c_{-}\right)=\sum_{\substack{1 \leq m \leq n \\ 0=a_{0}<a_{1}<\ldots a_{m}=n}} S\left(\beta_{1}, \beta_{2}, \ldots, \beta_{m} ; c_{+}, c_{-}\right) \cdot \prod_{j=1}^{m} \frac{1}{\left(a_{j}-a_{j-1}\right) !} .
$$

Now, let $j_{*}$ be the packet in which the vector $\left(1, N_{i_{*}}\right)$ lies. In view of $(5.18)$, the factor $S\left(\beta_{1}, \beta_{2}, \ldots, \beta_{m} ; c_{+}, c_{-}\right)$vanishes unless $j_{*}=1$ (which happens if $i_{*}=1$ ) or $j_{*}=2$ (which happens whenever $i_{*}>1$ and we group all the vectors $\left(0, N_{1}\right), \ldots,\left(0, N_{i_{*}-1}\right)$ in a single packet). In either case, the contraction of the head set is trivial, and we are left with

$$
U\left(\left\{N_{i}\right\}, i_{*} ; c_{+}, c_{-}\right)=\frac{1}{\left(i_{*}-1\right) !} \sum_{\left\{p_{r}\right\}} \frac{(-1)^{\operatorname{len}(p)}}{\prod_{r} p_{r} !},
$$


where the sum runs over ordered partitions of the $n-i_{*}$ elements in the tail set, i.e. integer sequences $\left\{p_{r}, 1 \leq r \leq \operatorname{len}(p)\right\}, p_{r} \geq 1$ such that $\sum_{r} p_{r}=n-i_{\star}$. This evaluates, for all $i_{*} \geq 1$, to the binomial coefficient

$$
U\left(\left\{N_{i}\right\}, i_{*} ; c_{+}, c_{-}\right)=\frac{(-1)^{n-i_{*}}}{\left(i_{*}-1\right) !\left(n-i_{*}\right) !},
$$

a result which is in particular independent of the partition $\left\{N_{i}\right\}$.

Now, we turn to the sum over graphs $g$. Due to the Landau factors $\left\langle\alpha_{i}, \alpha_{j}\right\rangle$ in (5.5), the only contributing graph is a tree rooted at $i_{*}$, with leaves $1, \ldots, i_{*}-1, i_{*}+1, \ldots, n$. The Landau factor is then given by

$$
\mathcal{L}=\prod_{i=1}^{i_{*}-1}\left\langle\left(0, N_{i}\right),\left(1, N_{i_{*}}\right)\right\rangle \prod_{i=i_{*}+1}^{n}\left\langle\left(1, N_{i_{*}}\right),\left(0, N_{i}\right)\right\rangle .
$$

Taking into account the additional factor $1 / 2^{n-1}$, we thus arrive at

$$
\begin{gathered}
\bar{\Omega}^{-}(1, N)=\sum_{n \geq 1} \sum_{\substack{1 \leq i_{*} \leq n \\
\sum_{1 \leq i \leq n} N_{i}=N}} \frac{(-1)^{n-1}(-1)^{i_{*}-1+\sum_{i \neq i_{*}} N_{i}}(-1)^{n-i_{*}} \gamma_{12}^{n-1}}{2^{n-1}\left(i_{*}-1\right) !\left(n-i_{*}\right) !} \\
\times \bar{\Omega}^{+}\left(1, N_{i_{*}}\right) \prod_{i \neq i_{*}} N_{i} \bar{\Omega}^{+}\left(0, N_{i}\right) .
\end{gathered}
$$

Plugging this expression in the partition function (4.11), we can easily carry out the sum over $N_{i}$ and obtain

$$
\bar{Z}^{-}(1, q)=\bar{Z}^{+}(1, q) \sum_{n \geq 1} \sum_{1 \leq i_{*} \leq n} \frac{\left[\log Z_{\mathrm{halo}}\left(\gamma_{1}, q\right)\right]^{n-1}}{2^{n-1}\left(i_{*}-1\right) !\left(n-i_{*}\right) !}
$$

where $Z_{\text {halo }}\left(\gamma_{1}, q\right)$ is the same function introduced in (4.30). The sum over $i_{*}$ leads to

$$
\bar{Z}^{-}(1, q)=\bar{Z}^{+}(1, q) \sum_{n \geq 1} \frac{\left[\log Z_{\text {halo }}\left(\gamma_{1}, q\right)\right]^{n-1}}{(n-1) !}
$$

and the sum over $n$ finally leads to

$$
\bar{Z}^{-}(1, q)=\bar{Z}^{+}(1, q) Z_{\text {halo }}\left(\gamma_{1}, q\right)
$$

Finally we note that the derivation given above can be simplified using the Boltzmann gas picture in which we analyze identical particle contribution to wall-crossing as a limit of non-identical particle contribution and then include an extra symmetry factor $1 / N$ ! for $N$ identical particles. To see how this works, we consider the case where we have $(N+1)$ different $\alpha_{i}$ 's satisfying $\alpha_{i j}>0$ for $i<j$, with the understanding that we shall eventually take the limit in which the first $N \alpha_{i}$ 's approach $\gamma_{2}$ or its multiple and $\alpha_{N+1}$ approaches $\gamma_{1}$. Now the $S$ and the $U$ factors coincide as in $\S 5.3$. Furthermore since eventually we shall take the limit in which the first $N \alpha_{i}$ 's coincide, the requirement of a non-vanishing $\mathcal{L}$ tells us that only trees which contribute are those in which $\alpha_{N+1}$ is connected to all the 
other $\alpha_{i}$ 's. We can still choose the direction of the arrows arbitrarily. Let us consider a configuration in which $m$ of the arrows are directed towards $\alpha_{N+1}$ and $n=N-m$ are directed away from it. In this case the arrows go from the nodes 1 to $m$ towards the central node $(m+1)$ and from the central node to the nodes $(m+2)$ to $(N+1)$. The Landau factor associated with these nodes is $(-1)^{m} \prod_{i=1}^{N}\left\langle\alpha_{N+1}, \alpha_{i}\right\rangle$. Next we need to assign the $\alpha_{i}$ 's to the different nodes. First of all there are $\left(\begin{array}{c}N \\ m\end{array}\right)$ ways of deciding which of the $\alpha_{i}$ 's will be assigned to the first $m$ nodes. Once this is done there is no further freedom of rearranging the $\alpha_{i}$ 's among the first $m$ nodes or the last $(N-m)$ nodes; in order that $U=S$ does not vanish, the $\alpha_{i}$ 's must be arranged in a clockwise order among the first $m$ nodes and anti-clockwise order among the last $(N-m)$ nodes. In this arrangement the possibility $(a)$ is realized $(N-m)$ times and the possibility $(b)$ is realized $m$ times. Hence the corresponding $U$ is given by $(-1)^{N-m}$. Substituting these into eq, (5.13) we now get

$$
g\left(\left\{\alpha_{i}\right\}\right)=2^{-N}(-1)^{\sum_{i<j}\left\langle\alpha_{i}, \alpha_{j}\right\rangle} \sum_{m=0}^{N}\left(\begin{array}{l}
N \\
m
\end{array}\right) \prod_{i=1}^{N}\left\langle\alpha_{N+1}, \alpha_{i}\right\rangle=(-1)^{\sum_{i<j}\left\langle\alpha_{i}, \alpha_{j}\right\rangle} \prod_{i=1}^{N}\left\langle\alpha_{N+1}, \alpha_{i}\right\rangle .
$$

If we now take the set $\left(\alpha_{1}, \ldots, \alpha_{N}\right)$ to contain $m_{l}$ copies of $l \gamma_{2}$ with $\sum l m_{l}=N$ then, including the symmetry factor $\prod_{l} 1 / m_{l}$ !, we get the coefficient of the $\bar{\Omega}^{+}\left(\gamma_{1}\right) \prod_{l} \bar{\Omega}^{+}\left(l \gamma_{2}\right)^{m_{l}}$ term to be

$$
\prod_{l} \frac{1}{m_{l} !}\left[(-1)^{l \gamma_{12}}\left(l \gamma_{12}\right)\right]^{m_{l}}
$$

which is the desired result.

\section{Acknowledgments}

We are grateful to D. Joyce and J. Stoppa for useful correspondence. J.M. thanks the LPTHE for hospitality during part of this work. The work of J.M. is partially supported by ANR grant BLAN06-3-137168. A.S. acknowledges the support of Chaires Internationales de Recherche Blaise Pascal during his stay at LPTHE where the work began, of the J. C. Bose fellowship of the Department of Science and Technology, India and of the project 11-R\& D-HRI-5.02-0304.

Note added in proof. In the sequel to this work [59], we derive the multiplicative renormalisation postulated above eq. (3.47) from the Atiyah-Bott Lefschetz fixed point formula for the equivariant index of the Dirac operator on $\mathcal{M}_{n}$. The main results of the present work are reviewed in the proceedings [60].

\section{A Wall crossing formulæ in special cases}

In this appendix we give explicit wall-crossing formulæ in some special cases. These cases illustrate the general results of $\S 4$ and $\S 5$, and serve as tests of the equivalence of the JS and KS wall-crossing formulæ. For brevity we state the results for the classical rational 
invariants $\bar{\Omega}$, but the same formulae also hold for the motivic rational invariants with the replacement (4.69). For notational convenience we shall denote $\bar{\Omega}^{ \pm}\left(M \gamma_{1}+N \gamma_{2}\right)$ by $\bar{\Omega}^{ \pm}(M, N)$. The result for $(M, N)=(2,2)$ below is in agreement with [29], Eq (2.13).

$$
\begin{aligned}
& \Delta \bar{\Omega}(1,2)=\kappa\left(2 \gamma_{12}\right) \bar{\Omega}^{+}(0,2) \bar{\Omega}^{+}(1,0) \\
& +\frac{1}{2}\left[\kappa\left(\gamma_{12}\right]\right)^{2}\left[\bar{\Omega}^{+}(0,1)\right]^{2} \bar{\Omega}^{+}(1,0)+\kappa\left(\gamma_{12}\right) \bar{\Omega}^{+}(0,1) \bar{\Omega}^{+}(1,1) \\
& \Delta \bar{\Omega}(2,2)=\kappa\left(4 \gamma_{12}\right) \bar{\Omega}^{+}(0,2) \bar{\Omega}^{+}(2,0)+\kappa\left(2 \gamma_{12}\right)\left[\bar{\Omega}^{+}(1,0) \bar{\Omega}^{+}(1,2)+\bar{\Omega}^{+}(0,1) \bar{\Omega}^{+}(2,1)\right] \\
& +\kappa\left(\gamma_{12}\right) \kappa\left(2 \gamma_{12}\right) \bar{\Omega}^{+}(0,1) \bar{\Omega}^{+}(1,0) \bar{\Omega}^{+}(1,1)+\frac{1}{4}\left[\kappa\left(\gamma_{12}\right)\right]^{2} \kappa\left(2 \gamma_{12}\right) \bar{\Omega}^{+}(0,1)^{2} \bar{\Omega}^{+}(1,0)^{2} \\
& +\frac{1}{2}\left[\kappa\left(2 \gamma_{12}\right)\right]^{2}\left[\bar{\Omega}^{+}(2,0) \bar{\Omega}^{+}(0,1)^{2}+\bar{\Omega}^{+}(0,2) \bar{\Omega}^{+}(1,0)^{2}\right] \\
& \Delta \bar{\Omega}(1,3)=\kappa\left(3 \gamma_{12}\right) \bar{\Omega}^{+}(0,3) \bar{\Omega}^{+}(1,0)+\kappa\left(2 \gamma_{12}\right) \bar{\Omega}^{+}(0,2) \bar{\Omega}^{+}(1,1)+\kappa\left(\gamma_{12}\right) \bar{\Omega}^{+}(0,1) \bar{\Omega}^{+}(1,2) \\
& +\kappa\left(\gamma_{12}\right) \kappa\left(2 \gamma_{12}\right) \bar{\Omega}^{+}(0,1) \bar{\Omega}^{+}(0,2) \bar{\Omega}^{+}(1,0)+\frac{1}{2}\left[\kappa\left(\gamma_{12}\right)\right]^{2} \bar{\Omega}^{+}(0,1)^{2} \bar{\Omega}^{+}(1,1) \\
& +\frac{1}{6}\left[\kappa\left(\gamma_{12}\right)\right]^{3} \bar{\Omega}^{+}(0,1)^{3} \bar{\Omega}^{+}(1,0) \\
& \Delta \bar{\Omega}(2,3)=\kappa\left(\gamma_{12}\right) \bar{\Omega}^{+}(1,1) \bar{\Omega}^{+}(1,2)+\kappa\left(3 \gamma_{12}\right) \bar{\Omega}^{+}(1,0) \bar{\Omega}^{+}(1,3)+\kappa\left(6 \gamma_{12}\right) \bar{\Omega}^{+}(0,3) \bar{\Omega}^{+}(2,0) \\
& +\kappa\left(4 \gamma_{12}\right) \bar{\Omega}^{+}(0,2) \bar{\Omega}^{+}(2,1)+\kappa\left(2 \gamma_{12}\right) \bar{\Omega}^{+}(0,1) \bar{\Omega}^{+}(2,2) \\
& +\frac{1}{2}\left[\kappa\left(\gamma_{12}\right)\right]^{2} \bar{\Omega}^{+}(0,1) \bar{\Omega}^{+}(1,1)^{2}+\frac{1}{2}\left[\kappa\left(3 \gamma_{12}\right)\right]^{2} \bar{\Omega}^{+}(0,3) \bar{\Omega}^{+}(1,0)^{2} \\
& +\frac{1}{2}\left[\left[\kappa\left(\gamma_{12}\right)\right]^{2}+\left[\kappa\left(2 \gamma_{12}\right)\right]^{2}+\kappa\left(\gamma_{12}\right) \kappa\left(3 \gamma_{12}\right)\right] \bar{\Omega}^{+}(0,1) \bar{\Omega}^{+}(1,0) \bar{\Omega}^{+}(1,2) \\
& +\frac{1}{2}\left[\kappa\left(\gamma_{12}\right) \kappa\left(2 \gamma_{12}\right)+\kappa\left(\gamma_{12}\right) \kappa\left(4 \gamma_{12}\right)+\kappa\left(2 \gamma_{12}\right) \kappa\left(3 \gamma_{12}\right)\right] \bar{\Omega}^{+}(0,2) \bar{\Omega}^{+}(1,0) \bar{\Omega}^{+}(1,1) \\
& +\kappa\left(2 \gamma_{12}\right) \kappa\left(4 \gamma_{12}\right) \bar{\Omega}^{+}(0,1) \bar{\Omega}^{+}(0,2) \bar{\Omega}^{+}(2,0)+\frac{1}{2}\left[\kappa\left(2 \gamma_{12}\right)\right]^{2} \bar{\Omega}^{+}(0,1)^{2} \bar{\Omega}^{+}(2,1) \\
& +\frac{1}{4}\left(3\left[\kappa\left(\gamma_{12}\right)\right]^{3}+\kappa\left(3 \gamma_{12}\right)\left[\kappa\left(\gamma_{12}\right)\right]^{2}+\kappa\left(\gamma_{12}\right)\left[\kappa\left(2 \gamma_{12}\right)\right]^{2}\right)\left[\bar{\Omega}^{+}(0,1)\right]^{2} \bar{\Omega}^{+}(1,0) \bar{\Omega}^{+}(1,1) \\
& +\frac{1}{2} \kappa\left(\gamma_{12}\right) \kappa\left(2 \gamma_{12}\right)\left[\kappa\left(\gamma_{12}\right)+\kappa\left(3 \gamma_{12}\right)\right]\left[\bar{\Omega}^{+}(1,0)\right]^{2} \bar{\Omega}^{+}(0,1) \bar{\Omega}^{+}(0,2) \\
& +\frac{1}{6}\left[\kappa\left(2 \gamma_{12}\right)\right]^{3}\left[\bar{\Omega}^{+}(0,1)\right]^{3} \bar{\Omega}^{+}(2,0) \\
& +\frac{1}{12}\left[\kappa\left(\gamma_{12}\right)\right]^{3}\left[3 \kappa\left(\gamma_{12}\right)+\kappa\left(3 \gamma_{12}\right)\right] \bar{\Omega}^{+}(0,1)^{3} \bar{\Omega}^{+}(1,0)^{2}
\end{aligned}
$$




$$
\begin{aligned}
& \Delta \bar{\Omega}(2,4)=\left[\frac{1}{12} \kappa\left(2 \gamma_{12}\right) \kappa\left(\gamma_{12}\right)^{4}+\frac{1}{48} \kappa\left(4 \gamma_{12}\right) \kappa\left(\gamma_{12}\right)^{4}\right] \bar{\Omega}^{+}(0,1)^{4} \bar{\Omega}^{+}(1,0)^{2} \\
& +\frac{1}{12}\left[4 \kappa\left(2 \gamma_{12}\right) \kappa\left(\gamma_{12}\right)^{3}+\kappa\left(4 \gamma_{12}\right) \kappa\left(\gamma_{12}\right)^{3}+\kappa\left(2 \gamma_{12}\right)^{3} \kappa\left(\gamma_{12}\right)\right] \bar{\Omega}^{+}(0,1)^{3} \bar{\Omega}^{+}(1,0) \bar{\Omega}^{+}(1,1) \\
& +\left[\frac{1}{2} \kappa\left(2 \gamma_{12}\right)^{2} \kappa\left(\gamma_{12}\right)^{2}+\frac{1}{4} \kappa\left(2 \gamma_{12}\right) \kappa\left(4 \gamma_{12}\right) \kappa\left(\gamma_{12}\right)^{2}\right] \bar{\Omega}^{+}(0,1)^{2} \bar{\Omega}^{+}(0,2) \bar{\Omega}^{+}(1,0)^{2} \\
& +\frac{1}{4} \bar{\Omega}^{+}(0,1)^{2} \bar{\Omega}^{+}(1,1)^{2} \kappa\left(2 \gamma_{12}\right) \kappa\left(\gamma_{12}\right)^{2} \\
& +\left[\frac{1}{2} \kappa\left(2 \gamma_{12}\right) \kappa\left(\gamma_{12}\right)^{2}+\frac{1}{4} \kappa\left(4 \gamma_{12}\right) \kappa\left(\gamma_{12}\right)^{2}+\frac{1}{4} \kappa\left(2 \gamma_{12}\right)^{3}\right] \bar{\Omega}^{+}(0,1)^{2} \bar{\Omega}^{+}(1,0) \bar{\Omega}^{+}(1,2) \\
& +\left[\kappa\left(2 \gamma_{12}\right)^{2} \kappa\left(\gamma_{12}\right)+\kappa\left(2 \gamma_{12}\right) \kappa\left(4 \gamma_{12}\right) \kappa\left(\gamma_{12}\right)\right] \bar{\Omega}^{+}(0,1) \bar{\Omega}^{+}(0,2) \bar{\Omega}^{+}(1,0) \bar{\Omega}^{+}(1,1) \\
& +\kappa\left(2 \gamma_{12}\right) \kappa\left(\gamma_{12}\right) \bar{\Omega}^{+}(0,1) \bar{\Omega}^{+}(1,1) \bar{\Omega}^{+}(1,2)+\frac{1}{2} \kappa\left(4 \gamma_{12}\right)^{2} \bar{\Omega}^{+}(0,4) \bar{\Omega}^{+}(1,0)^{2} \\
& +\frac{1}{2}\left[\kappa\left(2 \gamma_{12}\right) \kappa\left(\gamma_{12}\right)+\kappa\left(4 \gamma_{12}\right) \kappa\left(\gamma_{12}\right)+\kappa\left(2 \gamma_{12}\right) \kappa\left(3 \gamma_{12}\right)\right] \bar{\Omega}^{+}(0,1) \bar{\Omega}^{+}(1,0) \bar{\Omega}^{+}(1,3) \\
& +\frac{1}{2}\left[\kappa\left(2 \gamma_{12}\right) \kappa\left(3 \gamma_{12}\right) \kappa\left(\gamma_{12}\right)+\kappa\left(3 \gamma_{12}\right) \kappa\left(4 \gamma_{12}\right) \kappa\left(\gamma_{12}\right)\right] \bar{\Omega}^{+}(0,1) \bar{\Omega}^{+}(0,3) \bar{\Omega}^{+}(1,0)^{2} \\
& +\frac{1}{2}\left[\kappa\left(6 \gamma_{12}\right) \kappa\left(\gamma_{12}\right)+\kappa\left(2 \gamma_{12}\right) \kappa\left(3 \gamma_{12}\right)+\kappa\left(3 \gamma_{12}\right) \kappa\left(4 \gamma_{12}\right)\right] \bar{\Omega}^{+}(0,3) \bar{\Omega}^{+}(1,0) \bar{\Omega}^{+}(1,1) \\
& +\frac{1}{24} \kappa\left(2 \gamma_{12}\right)^{4} \bar{\Omega}^{+}(0,1)^{4} \bar{\Omega}^{+}(2,0)+\frac{1}{6} \kappa\left(2 \gamma_{12}\right)^{3} \bar{\Omega}^{+}(0,1)^{3} \bar{\Omega}^{+}(2,1) \\
& +\frac{1}{2} \kappa\left(2 \gamma_{12}\right)^{2} \bar{\Omega}^{+}(0,2) \bar{\Omega}^{+}(1,1)^{2}+\frac{1}{2} \kappa\left(2 \gamma_{12}\right)^{2} \bar{\Omega}^{+}(0,1)^{2} \bar{\Omega}^{+}(2,2) \\
& +\frac{1}{2} \kappa\left(4 \gamma_{12}\right)^{2} \bar{\Omega}^{+}(0,2)^{2} \bar{\Omega}^{+}(2,0)+\kappa\left(2 \gamma_{12}\right) \bar{\Omega}^{+}(1,1) \bar{\Omega}^{+}(1,3)+\kappa\left(2 \gamma_{12}\right) \bar{\Omega}^{+}(0,1) \bar{\Omega}^{+}(2,3) \\
& +\frac{1}{4} \kappa\left(2 \gamma_{12}\right)^{2} \kappa\left(4 \gamma_{12}\right) \bar{\Omega}^{+}(0,2)^{2} \bar{\Omega}^{+}(1,0)^{2}+\frac{1}{2} \kappa\left(2 \gamma_{12}\right)^{2} \kappa\left(4 \gamma_{12}\right) \bar{\Omega}^{+}(0,1)^{2} \bar{\Omega}^{+}(0,2) \bar{\Omega}^{+}(2,0) \\
& +\kappa\left(4 \gamma_{12}\right) \bar{\Omega}^{+}(1,0) \bar{\Omega}^{+}(1,4)+\kappa\left(4 \gamma_{12}\right) \bar{\Omega}^{+}(0,2) \bar{\Omega}^{+}(2,2)+\kappa\left(6 \gamma_{12}\right) \bar{\Omega}^{+}(0,3) \bar{\Omega}^{+}(2,1) \\
& +\kappa\left(2 \gamma_{12}\right) \kappa\left(4 \gamma_{12}\right) \bar{\Omega}^{+}(0,2) \bar{\Omega}^{+}(1,0) \bar{\Omega}^{+}(1,2)+\kappa\left(2 \gamma_{12}\right) \kappa\left(4 \gamma_{12}\right) \bar{\Omega}^{+}(0,1) \bar{\Omega}^{+}(0,2) \bar{\Omega}^{+}(2,1) \\
& +\kappa\left(2 \gamma_{12}\right) \kappa\left(6 \gamma_{12}\right) \bar{\Omega}^{+}(0,1) \bar{\Omega}^{+}(0,3) \bar{\Omega}^{+}(2,0)+\kappa\left(8 \gamma_{12}\right) \bar{\Omega}^{+}(0,4) \bar{\Omega}^{+}(2,0) .
\end{aligned}
$$

To derive these results from the JS wall-crossing formula described in $\S 5$, we use results for the $U$ and $\mathcal{L}$ factors tabulated below. In these tables, the first column describes the total charge, the second column lists the allowed ordered decompositions and the third and the fourth columns give the $U$ and $\mathcal{L}$ factors introduced in $\S 5.1$. Note that the $U$ 
factor typically involves a sum of multiple S-factors representing possible partitioning of the constituents as in (5.8), while the $\mathcal{L}$ factor comes from a sum of several trees as in (5.9).

\begin{tabular}{|c|c|c|c|}
\hline$\gamma$ & $\left(\alpha_{i}\right)$ & $U$ & $\mathcal{L}$ \\
\hline \multirow[t]{3}{*}{$(1,2)$} & $\{(0,1),(0,1),(1,0)\}$ & $\frac{1}{2}$ & $\gamma$ \\
\hline & $\{(0,1),(1,0),(0,1)\}$ & -1 & $-\gamma_{12}^{2}$ \\
\hline & $\{(1,0),(0,1),(0,1)\}$ & $\frac{1}{2}$ & \\
\hline \multirow[t]{9}{*}{$(1,3)$} & $\{(0,1),(0,2),(1,0)\}$ & $\frac{1}{2}$ & $2 \gamma_{12}^{2}$ \\
\hline & $\{(0,1),(1,0),(0,2)\}$ & -1 & $-2 \gamma_{12}^{2}$ \\
\hline & $\{(0,2),(0,1),(1,0)\}$ & $\frac{1}{2}$ & $2 \gamma_{12}^{2}$ \\
\hline & $\{(0,2),(1,0),(0,1)\}$ & -1 & $-2 \gamma_{12}^{2}$ \\
\hline & $\{(1,0),(0,1),(0,2)\}$ & $\frac{1}{2}$ & $2 \gamma_{12}^{2}$ \\
\hline & $\{(1,0),(0,2),(0,1)\}$ & $\frac{1}{2}$ & $2 \gamma_{12}^{2}$ \\
\hline & $\{(0,1),(0,1),(1,1)\}$ & $\frac{1}{2}$ & \\
\hline & $\{(0,1),(1,1),(0,1)\}$ & -1 & $-\gamma_{12}^{2}$ \\
\hline & $\{(1,1),(0,1),(0,1)\}$ & $\frac{1}{2}$ & \\
\hline \multirow[t]{12}{*}{$(2,2)$} & $\{(0,2),(1,0),(1,0)\}$ & $\frac{1}{2}$ & $4 \gamma_{12}^{2}$ \\
\hline & $\{(1,0),(0,2),(1,0)\}$ & -1 & $-4 \gamma_{12}^{2}$ \\
\hline & $\{(1,0),(1,0),(0,2)\}$ & $\frac{1}{2}$ & $4 \gamma_{12}^{2}$ \\
\hline & $\{(0,1),(1,0),(1,1)\}$ & $-\frac{1}{2}$ & $-\gamma_{12}^{2}$ \\
\hline & $\{(0,1),(1,1),(1,0)\}$ & 1 & $3 \gamma_{12}^{2}$ \\
\hline & $\{(1,0),(0,1),(1,1)\}$ & $-\frac{1}{2}$ & $-\gamma_{12}^{2}$ \\
\hline & $\{(1,0),(1,1),(0,1)\}$ & 1 & $3 \gamma_{12}^{2}$ \\
\hline & $\{(1,1),(0,1),(1,0)\}$ & $-\frac{1}{2}$ & $-\gamma_{12}^{2}$ \\
\hline & $\{(1,1),(1,0),(0,1)\}$ & $-\frac{1}{2}$ & $-\gamma_{12}^{2}$ \\
\hline & $\{(0,1),(0,1),(2,0)\}$ & $\frac{1}{2}$ & $4 \gamma_{12}^{2}$ \\
\hline & $\{(0,1),(2,0),(0,1)\}$ & -1 & $-4 \gamma_{12}^{2}$ \\
\hline & $\{(2,0),(0,1),(0,1)\}$ & $\frac{1}{2}$ & $4 \gamma_{12}^{2}$ \\
\hline
\end{tabular}

\begin{tabular}{|c|c|c|c|}
\hline \multirow{2}{*}{\begin{tabular}{|c|}
$\gamma$ \\
$(2,3)$
\end{tabular}} & $\left(\alpha_{i}\right)$ & $U$ & $\mathcal{L}$ \\
\hline & $\{(0,3),(1,0),(1,0)\}$ & $\frac{1}{2}$ & $9 \gamma_{1}^{2}$ \\
\hline & $\{(1,0),(0,3),(1,0)\}$ & -1 & -9 \\
\hline & $\{(1,0),(1,0),(0,3)\}$ & $\frac{1}{2}$ & $9 \gamma_{12}^{2}$ \\
\hline & $\{(0,2),(1,0),(1,1)\}$ & 0 & \\
\hline & $\{(0,2),(1,1),(1,0)\}$ & 1 & \\
\hline & $\{(1,0),(0,2),(1,1)\}$ & -1 & $-4 \gamma$ \\
\hline & $\{(1,0),(1,1),(0,2)\}$ & 1 & \\
\hline & $\{(1,1),(0,2),(1,0)\}$ & -1 & \\
\hline & $\{(1,1),(1,0),(0,2)\}$ & 0 & \\
\hline & $\{(0,1),(1,1),(1,1)\}$ & $\frac{1}{2}$ & \\
\hline & $\{(1,1),(0,1),(1,1)\}$ & -1 & $-\gamma$ \\
\hline & $\{(1,1),(1,1),(0,1)\}$ & $\frac{1}{2}$ & \\
\hline & $\{(0,1),(1,0),(1,2)\}$ & -1 & $-3 \gamma$ \\
\hline & $\{(0,1),(1,2),(1,0)\}$ & 1 & \\
\hline & $\{(1,0),(0,1),(1,2)\}$ & 0 & \\
\hline & $\{(1,0),(1,2),(0,1)\}$ & 1 & \\
\hline & $\{(1,2),(0,1),(1,0)\}$ & 0 & 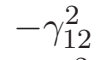 \\
\hline & $\{(1,2),(1,0),(0,1)\}$ & -1 & $-:$ \\
\hline & $\{(0,1),(0,2),(2,0)\}$ & $\frac{1}{2}$ & \\
\hline & $\{(0,1),(2,0),(0,2)\}$ & -1 & \\
\hline & $\{(0,2),(0,1),(2,0)\}$ & $\frac{1}{2}$ & \\
\hline & $\{(0,2),(2,0),(0,1)\}$ & -1 & $-8 \gamma_{12}^{2}$ \\
\hline & $\{(2,0),(0,1),(0,2)\}$ & $\frac{1}{2}$ & $8 \gamma_{12}^{2}$ \\
\hline & $\{(2,0),(0,2),(0,1)\}$ & $\frac{1}{2}$ & $8 \gamma_{1}^{2}$ \\
\hline & $\{(0,1),(0,1),(2,1)\}$ & $\frac{1}{2}$ & \\
\hline & $\{(0,1),(2,1),(0,1)\}$ & -1 & $-4 \gamma_{1}^{2}$ \\
\hline & $\{(2,1),(0,1),(0,1)\}$ & $\mid \overline{2}$ & \\
\hline
\end{tabular}

\begin{tabular}{|c|c|c|c|}
\hline$\gamma$ & $\left(\alpha_{i}\right)$ & $U$ & $\mathcal{L}$ \\
\hline$(2,3)$ & $\{(0,1),(0,1),(0,1),(1,0),(1,0)\}$ & $\frac{1}{12}$ & $-\gamma_{12}^{3}$ \\
& $\{(0,1),(0,1),(1,0),(0,1),(1,0)\}$ & 0 & $\gamma_{12}^{3}$ \\
& $\{(0,1),(0,1),(1,0),(1,0),(0,1)\}$ & $-\frac{1}{4}$ & $-4 \gamma_{12}^{3}$ \\
& $\{(0,1),(1,0),(0,1),(0,1),(1,0)\}$ & $-\frac{1}{2}$ & $-\gamma_{12}^{3}$ \\
& $\{(0,1),(1,0),(0,1),(1,0),(0,1)\}$ & 1 & $2 \gamma_{12}^{3}$ \\
& $\{(0,1),(1,0),(1,0),(0,1),(0,1)\}$ & $-\frac{1}{4}$ & 0 \\
& $\{(1,0),(0,1),(0,1),(0,1),(1,0)\}$ & $\frac{1}{3}$ & $\gamma_{12}^{3}$ \\
& $\{(1,0),(0,1),(0,1),(1,0),(0,1)\}$ & $-\frac{1}{2}$ & 0 \\
& $\{(1,0),(0,1),(1,0),(0,1),(0,1)\}$ & 0 & $-2 \gamma_{12}^{3}$ \\
& $\{(1,0),(1,0),(0,1),(0,1),(0,1)\}$ & $\frac{1}{12}$ & $4 \gamma_{12}^{3}$ \\
\hline
\end{tabular}




\begin{tabular}{|c|c|c|c|}
\hline$\gamma$ & $\left(\alpha_{i}\right)$ & $U$ & $\mathcal{L}$ \\
\hline \multirow[t]{4}{*}{$(1,3)$} & $\{(0,1),(0,1),(0,1),(1,0)\}$ & $\frac{1}{6}$ & $-\gamma_{12}^{3}$ \\
\hline & $\{(0,1),(0,1),(1,0),(0,1)\}$ & $-\frac{1}{2}$ & $\gamma_{12}^{3}$ \\
\hline & $\{(0,1),(1,0),(0,1),(0,1)\}$ & $\frac{1}{2}$ & $-\gamma_{12}^{3}$ \\
\hline & $\{(1,0),(0,1),(0,1),(0,1)\}$ & $-\frac{1}{6}$ & $\gamma_{12}^{3}$ \\
\hline \multirow[t]{6}{*}{$(2,2)$} & $\{(0,1),(0,1),(1,0),(1,0)\}$ & $\frac{1}{4}$ & $-4 \gamma_{12}^{3}$ \\
\hline & $\{(0,1),(1,0),(0,1),(1,0)\}$ & $-\frac{1}{2}$ & $2 \gamma_{12}^{3}$ \\
\hline & $\{(0,1),(1,0),(1,0),(0,1)\}$ & 0 & 0 \\
\hline & $\{(1,0),(0,1),(0,1),(1,0)\}$ & 0 & 0 \\
\hline & $\{(1,0),(0,1),(1,0),(0,1)\}$ & $\frac{1}{2}$ & $-2 \gamma_{12}^{3}$ \\
\hline & $\{(1,0),(1,0),(0,1),(0,1)\}$ & $-\frac{1}{4}$ & $4 \gamma_{12}^{3}$ \\
\hline \multirow[t]{28}{*}{2,3} & $\{(0,1),(0,2),(1,0),(1,0)\}$ & $\frac{1}{4}$ & $-12 \gamma_{12}^{3}$ \\
\hline & $\{(0,1),(1,0),(0,2),(1,0)\}$ & -1 & $8 \gamma_{12}^{3}$ \\
\hline & $\{(0,1),(1,0),(1,0),(0,2)\}$ & $\frac{1}{2}$ & $-4 \gamma_{12}^{3}$ \\
\hline & $\{(0,2),(0,1),(1,0),(1,0)\}$ & $\frac{1}{4}$ & $-12 \gamma_{12}^{3}$ \\
\hline & $\{(0,2),(1,0),(0,1),(1,0)\}$ & 0 & $4 \gamma_{12}^{3}$ \\
\hline & $\{(0,2),(1,0),(1,0),(0,1)\}$ & $-\frac{1}{2}$ & $4 \gamma_{12}^{3}$ \\
\hline & $\{(1,0),(0,1),(0,2),(1,0)\}$ & $\frac{1}{2}$ & 0 \\
\hline & $\{(1,0),(0,1),(1,0),(0,2)\}$ & 0 & $-4 \gamma_{12}^{3}$ \\
\hline & $\{(1,0),(0,2),(0,1),(1,0)\}$ & $-\frac{1}{2}$ & 0 \\
\hline & $\{(1,0),(0,2),(1,0),(0,1)\}$ & 1 & $-8 \gamma_{12}^{3}$ \\
\hline & $\{(1,0),(1,0),(0,1),(0,2)\}$ & $-\frac{1}{4}$ & $12 \gamma_{12}^{3}$ \\
\hline & $\{(1,0),(1,0),(0,2),(0,1)\}$ & $-\frac{1}{4}$ & $12 \gamma_{12}^{3}$ \\
\hline & $\{(0,1),(0,1),(1,0),(1,1)\}$ & 0 & 0 \\
\hline & $\{(0,1),(0,1),(1,1),(1,0)\}$ & $\frac{1}{2}$ & $-8 \gamma_{12}^{3}$ \\
\hline & $\{(0,1),(1,0),(0,1),(1,1)\}$ & -1 & $2 \gamma_{12}^{3}$ \\
\hline & $\{(0,1),(1,0),(1,1),(0,1)\}$ & 1 & $-4 \gamma_{12}^{3}$ \\
\hline & $\{(0,1),(1,1),(0,1),(1,0)\}$ & 0 & $2 \gamma_{12}^{3}$ \\
\hline & $\{(0,1),(1,1),(1,0),(0,1)\}$ & -1 & $4 \gamma_{12}^{3}$ \\
\hline & $\{(1,0),(0,1),(0,1),(1,1)\}$ & $\frac{1}{2}$ & 0 \\
\hline & $\{(1,0),(0,1),(1,1),(0,1)\}$ & 0 & $-2 \gamma_{12}^{3}$ \\
\hline & $\{(1,0),(1,1),(0,1),(0,1)\}$ & $-\frac{1}{2}$ & $8 \gamma_{12}^{3}$ \\
\hline & $\{(1,1),(0,1),(0,1),(1,0)\}$ & $-\frac{1}{2}$ & 0 \\
\hline & $\{(1,1),(0,1),(1,0),(0,1)\}$ & 1 & $-2 \gamma_{12}^{3}$ \\
\hline & $\{(1,1),(1,0),(0,1),(0,1)\}$ & 0 & 0 \\
\hline & $\{(0,1),(0,1),(0,1),(2,0)\}$ & $\frac{1}{6}$ & $-8 \gamma_{12}^{3}$ \\
\hline & $\{(0,1),(0,1),(2,0),(0,1)\}$ & $-\frac{1}{2}$ & $8 \gamma_{12}^{3}$ \\
\hline & $\{(0,1),(2,0),(0,1),(0,1)\}$ & $\frac{1}{2}$ & $-8 \gamma_{12}^{3}$ \\
\hline & $\{(2,0),(0,1),(0,1),(0,1)\}$ & $-\frac{1}{6}$ & $8 \gamma_{12}^{3}$ \\
\hline
\end{tabular}

(A.7) 


\section{B D6-D0 bound states}

In this subsection, we test and apply the wall-crossing formula on generalized DT-invariants for dimension zero sheaves on a Calabi-Yau threefold $\mathcal{X}$, for which many results are already known in the literature $[11,42,43,52]$.

The stability conditions for coherent sheaves on $\mathcal{X}$ depend on the complexified Kähler moduli $t^{a}=B^{a}+\mathrm{i} J^{a}$. The holomorphic central charge $Z_{\gamma}$ is given, in the large $J$ limit, by $Z_{\gamma}=-\int_{\mathcal{X}} e^{-t} \sqrt{\operatorname{Td}(\mathcal{X})} \wedge \gamma$. Let us denote by $\Omega(r, n ; t)$ the generalized DT-invariant for a sheaf of rank $r$, vanishing first and second Chern class, and third Chern class $n$. Physically, $\Omega(r, n)$ counts the number of bound states of $r$ D6-branes and $n$ D0-branes, with charge vector $\gamma=r \gamma_{1}+n \gamma_{2}, \gamma_{12}=-1$.

It is known that for infinite volume and small $B$-field, a configuration of $r \geq 1$ D6branes and $n \geq 1 \mathrm{D} 0$ branes do not form any bound state [53]. Moreover, there are no bound states of $r>1$ D6-branes, while $n \geq 1$ D0 bind into precisely $|\chi|$ bosonic (fermionic) bound states for negative (positive) $\chi$, where $\chi$ is the Euler number of $\mathcal{X}$. Thus the only non-vanishing DT invariants in this chamber are $[11,14]$

$$
\Omega^{+}(1,0)=1, \quad \Omega^{+}(0, n)=-\chi \quad(n>0),
$$

where we have used the notation $\Omega^{ \pm}(m, n)=\Omega^{ \pm}\left(m \gamma_{1}+n \gamma_{2}\right)$. Similarly, the motivic invariants are given by

$$
\Omega_{\mathrm{ref}}^{+}(1,0, y)=1, \quad \Omega_{\mathrm{ref}}^{+}(0, n, y)=-P(y) / y^{3} \quad(n>0),
$$

where $P(y)=1+b_{2} y^{2}-b_{3} y^{3}+b_{2} y^{4}+y^{6}$ is the Poincaré polynomial of $\mathcal{X}$, such that (B.2) reduces to (B.1) in the classical limit $y \rightarrow 1$.

By increasing the magnitude of the $B$-field, one reaches the wall of marginal stability $\mathcal{P}\left(\gamma_{1}, \gamma_{2}\right)$. We refer to the chamber across this wall as the 'DT' chamber. We shall obtain the motivic DT invariants in this chamber for $r \leq 3$ by applying the formulæ derived in section 4 , suitably generalized to the motivic case according to the discussion in section 4.7.

To apply the semi-primitive wall-crossing formulæ and its higher order generalizations, it is useful to introduce partition functions

$$
Z_{\mathrm{ref}}^{ \pm}(M, q, y)=\sum_{N=0}^{\infty} \Omega_{\mathrm{ref}}^{ \pm}\left(M \gamma_{1}+N \gamma_{2}, y\right) q^{N}, \quad \bar{Z}_{\mathrm{ref}}^{ \pm}(M, q, y)=\sum_{N=0}^{\infty} \bar{\Omega}_{\mathrm{ref}}^{ \pm}\left(M \gamma_{1}+N \gamma_{2}, y\right) q^{N}
$$

for fixed value of $M$. These two objects are related by

$$
\begin{aligned}
& \bar{Z}_{\mathrm{ref}}^{ \pm}(M, q, y)=\sum_{d \mid M} \frac{y-y^{-1}}{d\left(y^{d}-y^{-d}\right)} Z_{\mathrm{ref}}^{ \pm}\left(M / d, q^{d}, y^{d}\right), \\
& Z_{\mathrm{ref}}^{ \pm}(M, q, y)=\sum_{d \mid M} \mu(d) \frac{\left(y-y^{-1}\right.}{d\left(y^{d}-y^{-d}\right)} \bar{Z}_{\mathrm{ref}}^{ \pm}\left(M / d, q^{d}, y^{d}\right) .
\end{aligned}
$$


In the chamber $c_{+}$, the modified partition functions (B.3) are simply

$$
\bar{Z}_{\mathrm{ref}}^{+}(1, q, y)=1, \quad \bar{Z}_{\mathrm{ref}}^{+}(2, q, y)=\frac{y}{2\left(1+y^{2}\right)}, \quad \bar{Z}_{\mathrm{ref}}^{+}(3, q, y)=\frac{y^{2}}{3\left(1+y^{2}+y^{4}\right)} .
$$

Moreover, the partition function of the halo degeneracies follows from (4.73),

$$
\begin{gathered}
Z_{\text {halo }}\left(\gamma_{1}, q, y\right)=\prod_{k=1}^{\infty} \prod_{j=1}^{k}\left\{\left(1-(-q)^{k} y^{2 j-k+2}\right)^{-1}\left(1-(-q)^{k} y^{2 j-k}\right)^{-b_{2}}\left(1-(-q)^{k} y^{2 j-1-k}\right)^{b_{3}}\right. \\
\left.\left(1-(-q)^{k} y^{k-2 j}\right)^{-b_{2}}\left(1-(-q)^{k} y^{k-2 j-2}\right)^{-1}\right\}
\end{gathered}
$$

where we have made a change of variable $j \rightarrow k+1-j$ in the last two terms to make the $y \rightarrow y^{-1}$ symmetry manifest. Applying (4.71), we immediately obtain the partition function of the motivic invariants with $r=1$,

$$
Z_{\text {ref }}^{-}(1, q, y)=\bar{Z}_{\text {ref }}^{-}(1, q, y)=Z_{\text {halo }}\left(\gamma_{1}, q, y\right)
$$

In the classical limit $y \rightarrow 1$, this reduces to

$$
Z^{-}(1, q)=\bar{Z}^{-}(1, q)=[M(-q)]^{\chi}
$$

where $M(q)=\prod_{k=1}^{\infty}\left(1-q^{k}\right)^{-k}$ is the Mac-Mahon function [54-57]. For comparison with the higher rank formulæ of $[42,43]$ below, it is useful to note that the expansion of this formula in powers of $\chi$ reads

$$
e^{-\chi \sum_{k=1}^{\infty} k \log \left(1-(-q)^{k}\right)}=1+\sum_{p=1}^{\infty} \frac{\chi^{p}}{p !} \prod_{i=1}^{p} \sum_{k_{i}=1}^{\infty} \sum_{n_{i}=1}^{\infty} \frac{k_{i}}{n_{i}}(-q)^{k_{i} n_{i}}
$$

For $r=2$, we have

$$
Z_{\mathrm{ref}}^{-}(2, q, y)=\bar{Z}_{\mathrm{ref}}^{-}(2, q, y)-\frac{y}{2\left(1+y^{2}\right)} \bar{Z}_{\mathrm{ref}}^{-}\left(1, q^{2}, y^{2}\right)
$$

We now use (4.34), (4.41) together with the replacement (4.69) to obtain

$$
\begin{aligned}
Z_{\mathrm{ref}}^{-}(2, q, y)= & \frac{y}{2\left(1+y^{2}\right)}\left[Z_{\text {halo }}\left(2 \gamma_{1}, q, y\right)-Z_{\text {halo }}\left(\gamma_{1}, q^{2}, y^{2}\right)\right] \\
& -\frac{1}{4} \sum_{n_{1}, n_{2}} \kappa\left(\left|n_{1}-n_{2}\right|, y\right) \Omega_{\mathrm{ref}}^{-}\left(1, n_{1}, y\right) \Omega_{\mathrm{ref}}^{-}\left(1, n_{2}, y\right) q^{n_{1}+n_{2}} .
\end{aligned}
$$

In the classical limit, this reduces to

$$
Z^{-}(2, q)=\frac{1}{4}[M(q)]^{2 \chi}-\frac{1}{4}\left[M\left(-q^{2}\right)\right]^{\chi}-\frac{1}{4} \sum_{n_{1}, n_{2}}(-1)^{n_{1}-n_{2}}\left|n_{1}-n_{2}\right| \Omega^{-}\left(1, n_{1}\right) \Omega^{-}\left(1, n_{2}\right) q^{n_{1}+n_{2}} .
$$


This agrees with [43], theorem 1.2 and [42], (2.9), who obtain

$$
\begin{aligned}
\Omega^{-}(2, n)= & \frac{1}{4} \delta_{n, 0}+\sum_{p=1}^{\infty} 2^{p-2} \frac{\chi^{p}}{p !} \sum_{\substack{k_{i}>0, n_{i}>0 \\
\sum_{i} k_{i} n_{i}=n}} \prod_{i=1}^{p} \frac{k_{i}}{n_{i}}-\frac{1}{4} \Omega^{-}(1, n / 2) \\
& -\frac{1}{4} \sum_{\substack{n_{1} \geq 0, n_{2} \geq 0 \\
n_{1}+n_{2}=n}}(-1)^{n_{1}-n_{2}}\left|n_{1}-n_{2}\right| \Omega^{-}\left(1, n_{1}\right) \Omega^{-}\left(1, n_{2}\right)
\end{aligned}
$$

where it is understood that the third term is zero if $n$ is odd.

For $r=3$, we use similarly

$$
Z_{\mathrm{ref}}^{-}(3, q, y)=\bar{Z}_{\mathrm{ref}}^{-}(3, q, y)-\frac{y^{2}}{3\left(1+y^{2}+y^{4}\right)} \bar{Z}_{\mathrm{ref}}^{-}\left(1, q^{3}, y^{3}\right)
$$

and (4.46), (4.53) to obtain

$$
\begin{aligned}
Z_{\mathrm{ref}}^{-}(3, q, y)= & \frac{y^{2}}{3\left(1+y^{2}+y^{4}\right)}\left[Z_{\text {halo }}\left(3 \gamma_{1}, q, y\right)-Z_{\text {halo }}\left(\gamma_{1}, q^{3}, y^{3}\right)\right] \\
& -\frac{1}{2} \sum_{n_{1}, n_{2}} \kappa\left(\left|n_{2}-2 n_{1}\right|, y\right) \Omega_{\mathrm{ref}}^{-}\left(1, n_{1}, y\right) \Omega_{\mathrm{ref}}^{-}\left(2, n_{2}, y\right) q^{n_{1}+n_{2}} \\
& -\frac{y}{4\left(1+y^{2}\right)} \sum_{n_{1}, n_{2}} \kappa\left(2\left|n_{2}-n_{1}\right|, y\right) \Omega_{\mathrm{ref}}^{-}\left(1, n_{1}, y\right) \Omega_{\mathrm{ref}}^{-}\left(1, n_{2}, y^{2}\right) q^{n_{1}+2 n_{2}} \\
& -\frac{1}{12} \sum_{n_{1}, n_{2}} \kappa\left(n_{1}-n_{2}, y\right)^{2}\left[\Omega_{\mathrm{ref}}^{-}\left(1, n_{1}, y\right)\right]^{2} \Omega_{\mathrm{ref}}^{-}\left(1, n_{2}, y\right) q^{2 n_{1}+n_{2}} \\
& -\sum_{n_{1}>n_{2}>n_{3}} \kappa\left(n_{1}, n_{2}, n_{3}, y\right) \Omega_{\mathrm{ref}}^{-}\left(1, n_{1}, y\right) \Omega_{\mathrm{ref}}^{-}\left(1, n_{2}, y\right) \Omega_{\mathrm{ref}}^{-}\left(1, n_{3}, y\right) q^{n_{1}+n_{2}+n_{3}}
\end{aligned}
$$

In the classical limit, this reduces to

$$
\begin{aligned}
Z^{-}(3, q)= & \frac{1}{9}[M(-q)]^{3 \chi}-\frac{1}{9}\left[M\left(-q^{3}\right)\right]^{\chi} \\
& -\frac{1}{2} \sum_{n_{1}, n_{2}}(-1)^{n_{2}-2 n_{1}}\left|n_{2}-2 n_{1}\right| \Omega^{-}\left(1, n_{1}\right) \Omega^{-}\left(2, n_{2}\right) q^{n_{1}+n_{2}} \\
& -\frac{1}{4} \sum_{n_{1}, n_{2}}\left|n_{2}-n_{1}\right| \Omega^{-}\left(1, n_{1}\right) \Omega^{-}\left(1, n_{2}\right) q^{n_{1}+2 n_{2}} \\
& -\frac{1}{12} \sum_{n_{1}, n_{2}}\left(n_{1}-n_{2}\right)^{2}\left[\Omega^{-}\left(1, n_{1}\right)\right]^{2} \Omega^{-}\left(1, n_{2}\right) q^{2 n_{1}+n_{2}} \\
& -\sum_{n_{1}>n_{2}>n_{3}} \kappa\left(n_{1}, n_{2}, n_{3}\right) \Omega^{-}\left(1, n_{1}\right) \Omega^{-}\left(1, n_{2}\right) \Omega^{-}\left(1, n_{3}\right) q^{n_{1}+n_{2}+n_{3}}
\end{aligned}
$$


This can be rewritten as in [42], eq. (2.12), after correcting the coefficient of the 3-body term from $1 / 4$ into $1 / 6$ in that equation:

$$
\begin{aligned}
\Omega^{-}(3, n)= & \frac{1}{9} \delta_{n, 0}+\sum_{p=1}^{\infty} 3^{p-2} \frac{\chi^{p}}{p !} \sum_{\substack{k_{i}>0, n_{i}>0 \\
k_{i} n_{i}=n}} \prod_{i=1}^{p} \frac{k_{i}}{n_{i}}-\frac{1}{9} \Omega^{-}(1, n / 3) \\
& -\frac{1}{2} \sum_{\substack{n_{1} \geq 0, n_{2} \geq 0 \\
n_{1}+n_{2}=n}}(-1)^{n_{2}-2 n_{1}}\left|n_{2}-2 n_{1}\right| \Omega^{-}\left(1, n_{1}\right) \Omega^{-}\left(2, n_{2}\right) \\
& -\frac{1}{4} \sum_{\substack{n_{1} \geq 0, n_{2} \geq 0 \\
2 n_{1}+n_{2}=n}}\left|n_{1}-n_{2}\right| \Omega^{-}\left(1, n_{1}\right) \Omega^{-}\left(1, n_{2}\right) \\
- & \frac{1}{12} \sum_{\substack{n_{1} \geq 0, n_{2} \geq 0 \\
2 n_{1}+n_{2}=n}}\left(n_{1}-n_{2}\right)^{2}\left[\Omega^{-}\left(1, n_{1}\right)\right]^{2} \Omega^{-}\left(1, n_{2}\right) \\
& -\frac{1}{6} \sum_{\substack{0 \leq n_{1}<n_{2}<n_{3} \\
n_{1}+n_{2}+n_{3}=n}}^{\Omega^{-}\left(1, n_{1}\right) \Omega^{-}\left(1, n_{2}\right) \Omega^{-}\left(1, n_{3}\right)}
\end{aligned}
$$

where it is understood that the third term vanishes if $n$ is not a multiple of 3 .

The classical invariants $\Omega^{-}(r, n)$ for low values of $(r, n)$ are summarized in the table below:

\begin{tabular}{|c|c|c|c|c|c|}
\hline$r \backslash n$ & 0 & 1 & 2 & 3 & 4 \\
\hline 0 & $\cdot$ & $-\chi$ & $-\chi$ & $-\chi$ & $-\chi$ \\
1 & 1 & $-\chi$ & $\frac{1}{2}\left(\chi^{2}+5 \chi\right)$ & $-\frac{1}{6}\left(\chi^{3}+15 \chi^{2}+20 \chi\right)$ & $\frac{1}{24}\left(\chi^{4}+30 \chi^{3}+155 \chi^{2}+126 \chi\right)$ \\
2 & 0 & 0 & $-\chi$ & $-\frac{1}{6}\left(\chi^{3}+15 \chi^{2}+20 \chi\right)$ & $-\frac{1}{12}\left(\chi^{4}+30 \chi^{3}+119 \chi^{2}+102 \chi\right)$ \\
3 & 0 & 0 & 0 & $-\chi$ & $\frac{1}{24}\left(\chi^{4}+30 \chi^{3}+155 \chi^{2}+126 \chi\right)$ \\
\hline
\end{tabular}

\begin{tabular}{|c|c|}
\hline$r \backslash n$ & 5 \\
\hline 0 & $-\chi$ \\
1 & $-\frac{1}{120} \chi\left(\chi^{4}+50 \chi^{3}+575 \chi^{2}+1630 \chi+624\right)$ \\
2 & $-\frac{1}{120} \chi\left(7 \chi^{4}+250 \chi^{3}+1925 \chi^{2}+3890 \chi+1248\right)$ \\
3 & $-\frac{1}{120} \chi\left(7 \chi^{4}+250 \chi^{3}+1925 \chi^{2}+3890 \chi+1248\right)$ \\
\hline
\end{tabular}

For low values of $(r, n)$, the motivic invariants can also be expressed directly in terms of the Poincaré polynomial of the $\mathrm{CY}$ threefold $\mathcal{X}$,

$$
\begin{aligned}
(-y)^{6} \Omega_{\mathrm{ref}}^{-}(1,2, y)= & \frac{1}{2}\left[P\left(y^{2}\right)+[P(y)]^{2}\right]+\left(y^{4}+y^{2}\right) P(y) \\
= & 1+\left(b_{2}+1\right) y^{2}-b_{3} y^{3}+\frac{1}{2}\left(b_{2}^{2}+5 b_{2}+2\right) y^{4}-\left(b_{2}+1\right) b_{3} y^{5} \\
& +\frac{1}{2} y^{6}\left(2 b_{2}^{2}+4 b_{2}+b_{3}^{2}-b_{3}+2\right)-\left(b_{2}+1\right) b_{3} y^{7}+\frac{1}{2}\left(b_{2}^{2}+5 b_{2}+2\right) y^{8} \\
& -b_{3} y^{9}+\left(b_{2}+1\right) y^{10}+y^{12}
\end{aligned}
$$




$$
\begin{aligned}
(-y)^{9} \Omega_{\mathrm{ref}}^{-}(1,3, y)= & \frac{1}{6}[P(y)]^{3}+\frac{1}{2} P(y) P\left(y^{2}\right)+\left(y^{4}+y^{2}\right)[P(y)]^{2} \\
& +\left(\frac{1}{3} P\left(y^{3}\right)+\left(y^{8}+y^{6}+y^{4}\right) P(y)\right) \\
(-y)^{13} \Omega_{\mathrm{ref}}^{-}(2,4, y)=\frac{1}{24}\left[12\left(y^{3}+y\right)^{2}[P(y)]^{3}+\left(y^{2}+1\right)[P(y)]^{4}\right. & \\
& +3\left(y^{2}+1\right)\left(2 P\left(y^{4}\right)+\left[P\left(y^{2}\right)\right]^{2}+4\left(y^{8}+y^{4}\right) P\left(y^{2}\right)\right) \\
& +6\left(y^{2}+1\right)\left(P\left(y^{2}\right)+6 y^{8}+4 y^{6}+6 y^{4}\right)[P(y)]^{2} \\
& +4\left(3\left(y^{6}+y^{2}\right) P\left(y^{2}\right)+2\left(\left(y^{2}+1\right) P\left(y^{3}\right)\right.\right. \\
& \left.\left.\left.+3\left(y^{8}+y^{6}+2 y^{4}+y^{2}+1\right) y^{6}\right)\right) P(y)\right] \\
(-y)^{17} \Omega_{\mathrm{ref}}^{-}(3,5, y)= & \frac{1}{30}\left(y^{4}+1\right)\left(6 P\left(y^{5}\right)+5 P\left(y^{2}\right) P\left(y^{3}\right)\right) \\
+ & \frac{1}{8}\left(y^{4}+y^{2}+1\right) P(y)\left(2 P\left(y^{4}\right)+P\left(y^{2}\right)^{2}\right) \\
+ & \frac{1}{6}\left(y^{2}+1\right) P(y)\left(\left(y^{2}+1\right) P(y)+2\left(y^{6}+y^{2}\right)\right) P\left(y^{3}\right) \\
& +\frac{1}{12} P(y) P\left(y^{2}\right)\left[\left(y^{4}+3 y^{2}+1\right) P(y)^{2}+6\left(y^{8}+2 y^{6}+2 y^{4}+y^{2}\right) P(y)\right. \\
& \left.+12\left(y^{8}+y^{6}+2 y^{4}+y^{2}+1\right) y^{4}\right] \\
+ & \frac{1}{120} P(y)\left[120\left(y^{2}+1\right)^{2}\left(y^{4}+y^{2}+1\right) y^{4} P(y)^{2}+\left(y^{4}+5 y^{2}+1\right) P(y)^{4}\right. \\
& +20\left(y^{8}+4 y^{6}+4 y^{4}+y^{2}\right) P(y)^{3} \\
& +240\left(y^{10}+2 y^{8}+3 y^{6}+3 y^{4}+2 y^{2}+1\right) y^{6} P(y) \\
& \left.+120\left(y^{12}+y^{10}+2 y^{8}+2 y^{6}+2 y^{4}+y^{2}+1\right) y^{8}\right]
\end{aligned}
$$

The motivic invariants $\Omega_{\mathrm{ref}}^{-}(r, n,-y)$ have an expansion in positive and negative powers of $y$ of the form:

$$
\sum_{n=-K}^{K} c_{n} y^{n},
$$

for some integer $K$ with

$$
c_{n}=c_{-n}, \quad(-1)^{n} c_{n} \geq 0, \quad c_{n} \in \mathbf{Z}, \quad(-1)^{n}\left(c_{n}-c_{n+2}\right) \geq 0 \quad \text { for } \quad n \geq 0 .
$$

These are required for consistency of the interpretation (2.26) for the refined index. In particular $(-1)^{n}\left(c_{n}-c_{n+2}\right)$ measures the total number of states with angular momentum $n / 2$ contributing to the index (without counting the angular momentum factor $(n+1)$ ). Thus our results provide strong support to the motivic wall-crossing formula. Furthermore in all examples the refined index seems to satisfy the symmetry property

$$
\Omega_{\text {ref }}^{-}(r, n, y)=\Omega_{\text {ref }}^{-}(n-r, n, y)
$$

when $0 \leq r \leq n$. We have tested this for all $r \leq 3, n-r \leq 3$. In the classical case, this was established in [52]. 


\section{Seiberg-Witten spectra and generalizations}

In certain models, such as Seiberg-Witten theories in four dimensions, there exists a chamber in parameter space where the spectrum consists of only very few BPS states (the monopole and dyon for Seiberg-Witten theory with $G=\mathrm{SU}(2)$ and no flavor), separated from a single wall of marginal stability from a weak coupling region where an infinite number of BPS states are present. In this section, we use the formulae derived in the text to compute the degeneracies of the low-lying BPS states in the weak coupling region, from the knowledge of the degeneracies in the strong coupling region.

Assume that in the region $c_{+}$, the spectrum consists only of two states $\gamma_{1}, \gamma_{2}$ with BPS degeneracies $\Omega^{+}\left(\gamma_{1}\right)=q, \Omega^{+}\left(\gamma_{2}\right)=p$, respectively. Using the formulæ derived in the text, we find that the BPS invariants $\Omega^{-}\left(M \gamma_{1}+N \gamma_{2}\right)$ in the region $c_{-}$are given, for $\min (M, N) \leq 3$ by
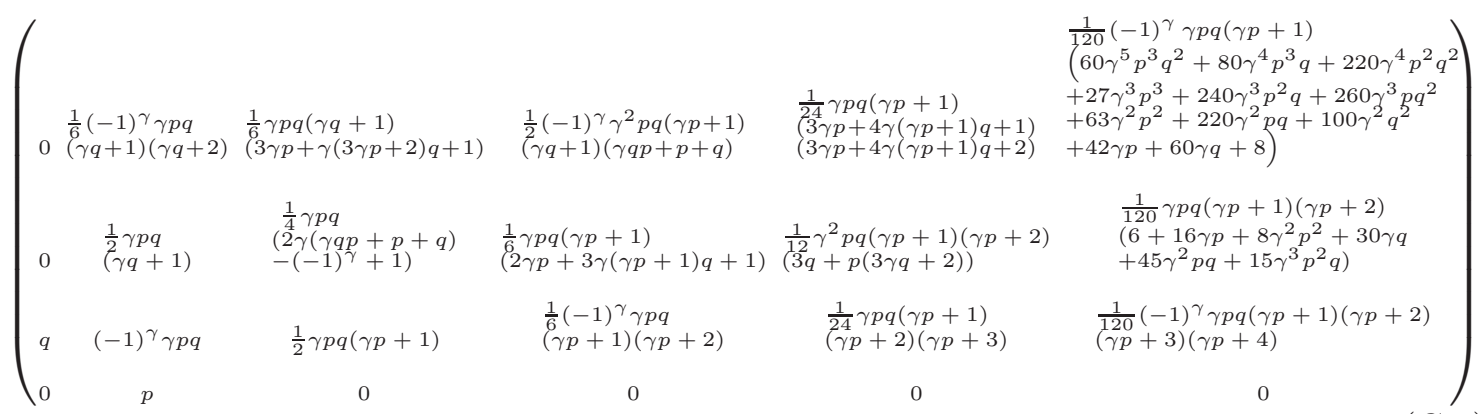

where $\gamma \equiv \gamma_{12}$ (In this table, $M$ increases from bottom to top, while $N$ increases from left to right). This table has been given for $\gamma<0$; as discussed in $\S 4.6$, for a general sign of $\gamma$ the result is given by replacing $\gamma \rightarrow-|\gamma|$ in this table. With this replacement the table is symmetric under $p \leftrightarrow q, M \leftrightarrow N$, and we can recover some of the results for $M=4$ and 5 from the corresponding results for $N=4$ and 5 .

We now compare the above table with some known results. For $(p, q, \gamma)=(1,1,-1)$, eq. (C.1) agrees with the pentagonal identity (4.58). For SU(2) Seiberg-Witten theories with $N_{f}=0,2,3$ flavors, the BPS spectrum in the strong coupling chamber [2-5] also falls into the case considered here, for suitable choices of $(p, q, \gamma)$ :

- for $N_{f}=0$, there is a single state of charge $\gamma_{1}=(2,1)^{19}$ and a single state of charge $\gamma_{2}=(0,-1)$, corresponding to $(p, q, \gamma)=(1,1,-2)$;

- for $N_{f}=2$, two states of charge $\gamma_{1}=(1,1)$ and two states of charge $\gamma_{2}=(0,-1)$ transforming respectively in the $(2,1)$ and $(1,2)$ representations of the $\mathrm{SO}(4)$ flavour group, corresponding to $(p, q, \gamma)=(2,2,-1)$;

- for $N_{f}=3$, four states of charge $\gamma_{1}=(0,1)$ and a single state of charge $\gamma_{2}=(1,-2)$ transforming respectively in the 4 and 1 representation of the $\mathrm{SO}(6)$ flavour group, corresponding to $(p, q, \gamma)=(1,4,-1)$.

\footnotetext{
${ }^{19}$ Here $\left(n_{e}, n_{m}\right)$ denotes a state carrying electric charge $n_{e}$ and magnetic charge $n_{m}$.
} 
Applying (C.1), we reproduce the low lying BPS states in the weak coupling chamber, as already demonstrated in $[17,35]$.

If instead the spectrum in $c_{+}$consists of three states $\gamma_{1}, \gamma_{2}, \gamma_{1}+\gamma_{2}$, with respective BPS degeneracies $p, q, r$, the spectrum in $c_{-}$is given by

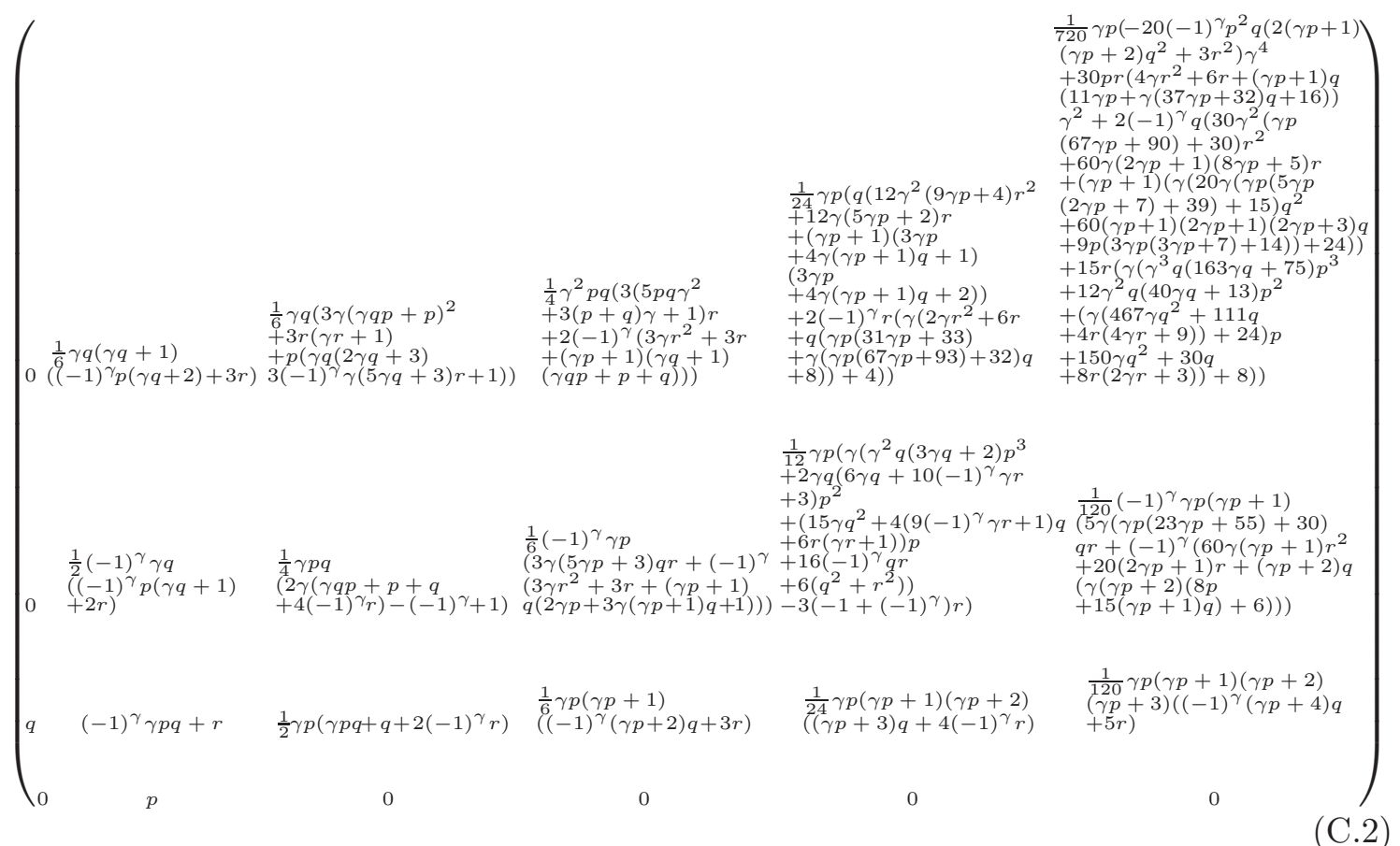

For $(p, q, r, \gamma)=(1,1,1,1)$ this reproduces again the pentagonal identity (4.58) (read backwards). For $(p, q, r, \gamma)=(1,1,1,-1)$ this now reproduces the weak coupling spectrum of the SU(2) Seiberg-Witten theory with $N_{f}=1$ flavor, whose strong coupling BPS spectrum consists of three states of unit degeneracy each, carrying charges $\gamma_{1}=(0,1), \gamma_{2}=(1,-1)$ and $\gamma_{1}+\gamma_{2}=(1,0)$.

\section{D $\mathrm{U}(\mathrm{N})$ quiver quantum mechanics from Boltzmann black hole halos}

In this appendix we shall show that our Boltzmann gas picture of multi-centered black hole system also makes a specific prediction on quiver quantum mechanics without oriented loops. Consider a multi-black hole configuration with $(n+N)$ centers, with the first $n$ centers carrying charges $\alpha_{1}, \ldots, \alpha_{n}$ and each of the last $N$ centers carrying charge $\beta_{0}$. We take all the $\alpha_{i}$ 's and $\beta_{0}$ to lie in a two dimensional plane, and assume, without any loss of generality, that $\alpha_{i j} \equiv\left\langle\alpha_{i}, \alpha_{j}\right\rangle>0$ for $i<j$. Suppose further that each center carries unit intrinsic index, i.e. $\Omega^{+}\left(\alpha_{i}\right)=\Omega^{+}\left(\beta_{0}\right)=1$, and that $\Omega^{+}\left(k \beta_{0}\right)=0$ for $k>1$. In this case the system is described by a $\mathrm{U}(1)^{n} \times \mathrm{U}(N)$ quiver quantum mechanics [8], and the refined index $\operatorname{Tr}(-y)^{2 J_{3}}$ of this quantum mechanics is given by

$$
(-y)^{-C_{\mathrm{U}(1)^{n} \times \mathrm{U}(N)}\left(\alpha_{1}, \ldots, \alpha_{n}, \beta_{0}\right)} P_{\mathrm{U}(1)^{n} \times \mathrm{U}(N)}\left(\alpha_{1}, \ldots, \alpha_{n}, \beta_{0}, y\right) .
$$

Here $P_{\mathrm{U}(1)^{n} \times \mathrm{U}(N)}\left(\alpha_{1}, \ldots, \alpha_{n}, \beta_{0}, y\right)$ is the Poincaré polynomial associated with a quiver quantum mechanics with $n+1$ nodes, with the first $n$-nodes carrying $\mathrm{U}(1)$ factors and the last node carrying a $\mathrm{U}(N)$ factor. Furthermore there are $\alpha_{i j}$ arrows from 
the $i$ 'th to the $j$ 'th node for $i<j,\left|\left\langle\alpha_{i}, \beta_{0}\right\rangle\right|$ arrows from the $i$ th node to the last node if $\left\langle\alpha_{i}, \beta_{0}\right\rangle>0$ and $\left|\left\langle\alpha_{i}, \beta_{0}\right\rangle\right|$ arrows from the last node to the $i$ th node if $\left\langle\alpha_{i}, \beta_{0}\right\rangle<0 . C_{\mathrm{U}(1)^{n} \times \mathrm{U}(N)}\left(\alpha_{1}, \ldots, \alpha_{n}, \beta_{0}\right)$ denotes the maximum power of $y^{2}$ in $P_{\mathrm{U}(1)^{n} \times \mathrm{U}(N)}\left(\alpha_{1}, \ldots, \alpha_{n}, \beta_{0}, y\right)$, signifying the maximum value of $2 J_{3}$ carried by the system. An explicit expression for the Poincaré polynomial of this quiver will be given in eq. (D.7) below. On the other hand we can also calculate the index using (1.3)-(1.6). First note that we have, from (1.3),

$$
\bar{\Omega}^{+}\left(l \beta_{0}\right)=\frac{y-y^{-1}}{l\left(y^{l}-y^{-l}\right)} .
$$

Using (1.5) we find that this configuration contributes

$$
\sum_{\substack{\left\{k_{l}\right\} \\ \sum k_{l}=N}} g_{\mathrm{ref}}\left(\alpha_{1}, \ldots, \alpha_{n}, \beta_{0} \times k_{1}, 2 \beta_{0} \times k_{2}, \ldots, y\right) \prod_{l}\left\{\frac{1}{k_{l} !} \bar{\Omega}^{+}\left(l \beta_{0}\right)^{k_{l}}\right\}
$$

to $\operatorname{Tr}(-y)^{2 J_{3}}$, where $g_{\text {ref }}$ is given by $(1.6)$ and the symbol $l \beta_{0} \times k_{l}$ means that the vector $l \beta_{0}$ is repeated $k_{l}$ times. To study the consequences of equating (D.3) to (D.1), note that the expression for $g_{\text {ref }}$ given in (1.6), after stripping off the first factor is nothing but the Poincaré polynomial of a quiver theory with only U(1) factors. This gives

$$
\begin{aligned}
& g_{\mathrm{ref}}\left(\alpha_{1}, \ldots, \alpha_{n}, \beta_{0} \times k_{1}, 2 \beta_{0} \times k_{2}, \cdots, y\right)=(-y)^{n+\sum_{l} k_{l}-1-\sum_{i<j} \alpha_{i j}-N \sum_{i}\left|\left\langle\alpha_{i}, \beta_{0}\right\rangle\right|} \\
& \times P_{\mathrm{U}(1)^{n} \times \mathrm{U}(1)^{k_{1}} \times \mathrm{U}(1)^{k_{2}} \times \cdots}\left(\alpha_{1}, \ldots, \alpha_{n}, \beta_{0} \times k_{1}, 2 \beta_{0} \times k_{2}, \cdots, y\right) .
\end{aligned}
$$

Using eqs.(D.1), (D.2) and (D.4) we get a direct relation between the Poincaré polynomials of a quiver carrying a $\mathrm{U}(N)$ factor and those carrying only $\mathrm{U}(1)$ factors:

$$
\begin{aligned}
& P_{\mathrm{U}(1)^{n} \times \mathrm{U}(N)}\left(\alpha_{1}, \ldots, \alpha_{n}, \beta_{0}, y\right)=\sum_{\substack{\left\{k_{l}\right\} \\
\sum l k_{l}=N}} P_{\mathrm{U}(1)^{n} \times \prod_{l} \mathrm{U}(1)^{k_{l}}}\left(\alpha_{1}, \ldots, \alpha_{n}, \beta_{0} \times k_{1}, 2 \beta_{0} \times k_{2}, \cdots, y\right)
\end{aligned}
$$

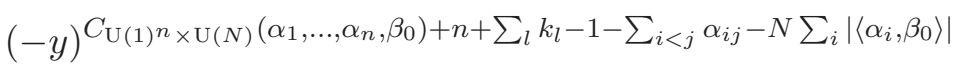

$$
\begin{aligned}
& \left\{\prod_{l} \frac{1}{k_{l} !}\left(\frac{y-y^{-1}}{l\left(y^{l}-y^{-l}\right)}\right)^{k_{l}}\right\}
\end{aligned}
$$

We could also derive a similar relation where the first $\mathrm{U}(1)^{n}$ factor is replaced by a product of $\mathrm{U}(M)$ factors by choosing among the set $\left(\alpha_{1}, \ldots, \alpha_{n}\right)$ identical charges. Let us use a shorthand notation where $P_{N_{1}, \ldots, N_{p}}\left(\beta_{1}, \ldots, \beta_{p}, y\right)$ denotes the Poincaré polynomial of a quiver with the $i$-th node having a $\mathrm{U}\left(N_{i}\right)$ factor and charge $\beta_{i}$ and furthermore arrange the 
$\beta_{i}$ 's such that $\beta_{i j}>0$ for $i>j$. Similarly $C_{N_{1}, \ldots, N_{p}}\left(\beta_{1}, \ldots, \beta_{p}\right)$ will denote the maximum power of $y^{2}$ in this polynomial. Then the generalization of (D.5) will take the form

$$
\begin{aligned}
& y^{-C_{N_{1}, \ldots, N_{p}}\left(\beta_{1}, \ldots, \beta_{p}\right)} P_{N_{1} \cdots N_{p}}\left(\beta_{1}, \ldots, \beta_{p}, y\right) \\
= & \sum_{\substack{\left\{k_{l}\right\} \\
\sum_{l}=N_{r}}} P_{N_{1}, \ldots, N_{r-1}, 1 \times k_{1}, 1 \times k_{2}, \cdots, N_{r+1}, \ldots, N_{p}}\left(\beta_{1}, \ldots, \beta_{r-1}, \beta_{r} \times k_{1}, 2 \beta_{r} \times k_{2}, \cdots, \beta_{r+1}, \ldots, \beta_{p}, y\right) \\
y^{-C_{N_{1}, \ldots, N_{r-1}, 1 \times k_{1}, 1 \times k_{2}, \cdots, N_{r+1}, \ldots, N_{p}}\left(\beta_{1}, \ldots, \beta_{r-1}, \beta_{r} \times k_{1}, 2 \beta_{r} \times k_{2}, \cdots, \beta_{r+1}, \ldots, \beta_{p}\right)} & \left\{\prod_{l} \frac{1}{k_{l} !}\left(\frac{y-y^{-1}}{l\left(y^{l}-y^{-l}\right)}(-1)^{l-1}\right)^{k_{l}}\right\}
\end{aligned}
$$

where we have substituted $y \rightarrow-y$, using the fact that the Poincaré polynomial of a quiver is an even function of $y$. By repeated use of (D.6) we can express the Poincaré polynomial of a quiver with $\mathrm{U}(N)$ factors in terms of the Poincaré polynomial of quivers with $\mathrm{U}(1)$ factors only.

We now test e.q. (D.6) against the Poincaré polynomial of a quiver quantum mechanics with $\mathrm{U}(N)$ factors but no oriented loops computed in [40] (see also [8]):

$$
\begin{aligned}
P_{N_{1}, \cdots, N_{p}}\left(\beta_{1}, \ldots, \beta_{p}, y\right)=\left(y^{2}-1\right)^{1-\sum_{i} N_{i}} y^{-\sum_{i} N_{i}\left(N_{i}-1\right)} & \\
& \sum_{\text {partitions }}(-1)^{s-1} y^{2 \sum_{a \leq b} \sum_{i<j} \beta_{i j} N_{i}^{b} N_{j}^{a}} \prod_{a, i}\left(\left[N_{i}^{a}, y\right] !\right)^{-1}, \\
C_{N_{1}, \cdots, N_{p}}\left(\beta_{1}, \ldots, \beta_{p}\right)= & \sum_{i<j} N_{i} N_{j} \beta_{i j}-\sum_{i} N_{i}^{2}+1
\end{aligned}
$$

where

$$
[N, y] \equiv \frac{y^{2 N}-1}{y^{2}-1}, \quad[N, y] ! \equiv[1, y][2, y] \ldots[N, y]
$$

and the sum over partitions in (D.7) runs over all ordered partitions of the vector $\sum_{i} N_{i} \beta_{i}$ into non-zero vectors $\left\{\sum_{i} N_{i}^{a} \beta_{i}, \quad a=1, \ldots, s\right\}$ for $s=1, \ldots, \sum_{i} N_{i}$, satisfying $\sum_{a} N_{a}^{i}=$ $N_{i}$ and $^{20}$

$$
\left\langle\sum_{a=1}^{b} \sum_{i=1}^{p} N_{i}^{a} \beta_{i}, \sum_{j=1}^{p} N_{j} \beta_{j}\right\rangle>0,
$$

for $b=1, \ldots, s-1$. If $C_{N_{1}, \ldots, N_{p}}\left(\beta_{1}, \ldots, \beta_{p}\right)$ given in (D.7) becomes negative then the Poincaré polynomial vanishes.

Based on (D.7), eq. (D.6) can be proven by collecting the terms multiplying a given partition on both sides of this equation and showing that they cancel. Consider for example a partition with $s$ vectors of the form $\sum_{i=1}^{p} N_{i}^{a} \beta_{i}$ for $a=1, \ldots, s$ and let $N_{r}^{a}=M_{a}$. Thus the $s$ vectors have the form $A_{1}+M_{1} \beta_{r}, A_{2}+M_{2} \beta_{r}, \ldots A_{s}+M_{s} \beta_{r}$ where $A_{1}, \ldots, A_{s}$ are linear combinations of the $\beta_{i}$ 's other than $\beta_{r}$. If we just focus on the contribution involving

\footnotetext{
${ }^{20}$ Normally this relation is written as a condition on the phase of $Z_{\sum_{a=1}^{b} \sum_{i} N_{i}^{a} \beta_{i}}$, but we have used (3.33) to expres this as a condition involving the symplectic products.
} 
the $\mathrm{U}\left(N_{r}\right)$ factor then the contribution to this particular partition from the left hand side of (D.6) contains a factor of

$$
\left(y^{2}-1\right)^{-N_{r}} y^{N_{r}} \prod_{a}\left(\left[M_{a}, y\right] !\right)^{-1},
$$

where we have made use of (D.7) and ignored the $y^{-\sum_{i<j} N_{i} N_{j} \beta_{i j}}$ term since it is a common factor on both sides of (D.6). On the right hand side this particular partition can come from many different terms. In particular $M_{a} \beta_{r}$ can arise as a sum of $k_{1}^{(a)}$ copies of $\beta_{r}, k_{2}^{(a)}$ copies of $2 \beta_{r}$ etc. with $M_{a}=\sum_{l} k_{l}^{(a)} l$. This in turn can arise from the $P_{N_{1}, \ldots, N_{r-1}, 1 \times k_{1}, 1 \times k_{2}, \cdots, N_{r+1}, \ldots, N_{p}}$ term if the $k_{l}^{(a)}$ 's satisfy $\sum_{a} k_{l}^{(a)}=k_{l}$. There is a combinatoric factor of $k_{l} ! / \prod_{a} k_{l}^{(a)}$ ! associated with different ways of choosing the $k_{l}^{(a)}$ copies of $l \beta_{r}$ out of $k_{l}$ copies of $l \beta_{r}$. Using (D.6) and (D.7) we see that the relevant factor on the right hand side is

$$
\begin{aligned}
& \sum_{\left\{k_{l}\right\}} \sum_{\left\{k_{l}^{(a)}\right\}} \frac{1}{\prod_{a, l} k_{l}^{(a)} !}\left(y^{2}-1\right)^{-\sum_{l} k_{l}} y^{\sum_{l} k_{l}}\left\{\prod_{l}\left(\frac{y-y^{-1}}{l\left(y^{l}-y^{-l}\right)}(-1)^{l-1}\right)^{k_{l}}\right\} . \\
& \sum_{l} k_{l}^{(a)} l=M_{a}, \sum_{a} k_{l}^{(a)}=k_{l}
\end{aligned}
$$

We can simplify this as follows. First of all using the relation $N_{r}=\sum_{l} k_{l} l$, we can express $\sum_{l} k_{l}$ in the exponents of $\left(y^{2}-1\right)$ and $y$ as $N_{r}-\sum_{l} k_{l}(l-1)=N_{r}-\sum_{l, a} k_{l}^{(a)}(l-1)$. Next the sum over $\left\{k_{l}\right\}$ and the restriction $\sum_{a} k_{l}^{(a)}=k_{l}$ can be removed if we replace all factors of $k_{l}$ by $\sum_{a} k_{l}^{(a)}$. Using (D.8) this allows us to express (D.11) as

$$
\left(y^{2}-1\right)^{-N_{r}} y^{N_{r}} \prod_{a}\left\{\sum_{\substack{k_{l}^{(a)} \\ \sum_{l} k_{l}^{(a)} l=M_{a}}}\left(y^{2}-1\right)^{\sum_{l} k_{l}^{(a)}(l-1)} \prod_{l}\left(\frac{(-1)^{l-1}}{[l, y] l}\right)^{k_{l}^{(a)}}\right\} .
$$

The equality of (D.10) and (D.12) is now reduced to the following identity:

$$
\frac{1}{[N, y] !} \stackrel{?}{=} \sum_{\substack{\left\{k_{l}\right\} \\ \sum_{l} l k_{l}=N}} \prod_{l} \frac{1}{k_{l} !}(-1)^{k_{l}(l-1)}\left(y^{2}-1\right)^{k_{l}(l-1)}\left(\frac{1}{[l, y] l}\right)^{k_{l}}
$$

To prove (D.13), consider the equivalent identity for partition functions

$$
\sum_{N=0}^{\infty} \frac{z^{N}}{[N, y] !} \stackrel{?}{=} \exp \left(\sum_{l=1}^{\infty} \frac{\left(1-y^{2}\right)^{l}}{1-y^{2 l}} \frac{z^{l}}{l}\right)
$$

Rewriting the r.h.s. as

$$
\exp \left(\sum_{k=0}^{\infty} \sum_{l=1}^{\infty} \frac{\left[z\left(1-y^{2}\right)\right]^{l} y^{2 l k}}{l}\right)=\prod_{k=0}^{\infty}\left(1-z\left(1-y^{2}\right) y^{2 k}\right)^{-1},
$$


we see that (D.13) follows from the known relation between the $q$-deformed exponential and the $q$-deformed Pochhammer symbol with $q=y^{2}$ (see e.g. [58]),

$$
\sum_{N=0}^{\infty} \frac{z^{N}}{[N, y] !}=(z(1-q) ; q)_{\infty}=\prod_{k=0}^{\infty}\left(1-z(1-q) q^{k}\right)^{-1}, \quad|q|<1 .
$$

Open Access. This article is distributed under the terms of the Creative Commons Attribution Noncommercial License which permits any noncommercial use, distribution, and reproduction in any medium, provided the original author(s) and source are credited.

\section{References}

[1] S. Cecotti and C. Vafa, On classification of $N=2$ supersymmetric theories, Commun. Math. Phys. 158 (1993) 569 [hep-th/9211097] [SPIRES].

[2] N. Seiberg and E. Witten, Monopole condensation, and confinement in $N=2$ supersymmetric Yang-Mills theory, Nucl. Phys. B 426 (1994) 19 [hep-th/9407087] [SPIRES].

[3] N. Seiberg and E. Witten, Monopoles, duality and chiral symmetry breaking in $N=2$ supersymmetric QCD, Nucl. Phys. B 431 (1994) 484 [hep-th/9408099] [SPIRES].

[4] F. Ferrari and A. Bilal, The strong-coupling spectrum of the Seiberg-Witten theory, Nucl. Phys. B 469 (1996) 387 [hep-th/9602082] [SPIRES].

[5] A. Bilal and F. Ferrari, Curves of marginal stability and weak and strong-coupling BPS spectra in $N=2$ supersymmetric QCD, Nucl. Phys. B 480 (1996) 589 [hep-th/9605101] [SPIRES].

[6] M.R. Douglas, D-branes, categories and $N=1$ supersymmetry, J. Math. Phys. 42 (2001) 2818 [hep-th/0011017] [SPIRES].

[7] F. Denef, Supergravity flows and D-brane stability, JHEP 08 (2000) 050 [hep-th/0005049] [SPIRES].

[8] F. Denef, Quantum quivers and Hall/hole halos, JHEP 10 (2002) 023 [hep-th/0206072] [SPIRES].

[9] B. Bates and F. Denef, Exact solutions for supersymmetric stationary black hole composites, hep-th/0304094 [SPIRES].

[10] F. Denef and G.W. Moore, Split states, entropy enigmas, holes and halos, hep-th/0702146 [SPIRES].

[11] M. Kontsevich and Y. Soibelman, Stability structures, motivic Donaldson-Thomas invariants and cluster transformations, arXiv:0811.2435.

[12] M. Kontsevich and Y. Soibelman, Motivic Donaldson-Thomas invariants: summary of results, arXiv:0910.4315 [SPIRES].

[13] D. Joyce, Configurations in abelian categories IV. Invariants and changing stability conditions, Adv. Math. 217 (2008) 125.

[14] D. Joyce and Y. Song, A theory of generalized Donaldson-Thomas invariants, arXiv: 0810.5645 [SPIRES].

[15] D. Joyce, Generalized Donaldson-Thomas invariants, arXiv:0910.0105 [SPIRES]. 
[16] E. Diaconescu and G.W. Moore, Crossing the wall: branes vs. bundles, arXiv:0706.3193 [SPIRES].

[17] D. Gaiotto, G.W. Moore and A. Neitzke, Four-dimensional wall-crossing via three-dimensional field theory, Commun. Math. Phys. 299 (2010) 163 [arXiv:0807.4723] [SPIRES].

[18] S. Alexandrov, B. Pioline, F. Saueressig and S. Vandoren, D-instantons and twistors, JHEP 03 (2009) 044 [arXiv:0812.4219] [SPIRES].

[19] D.L. Jafferis and G.W. Moore, Wall crossing in local Calabi Yau manifolds, arXiv:0810.4909 [SPIRES].

[20] S. Cecotti and C. Vafa, BPS wall crossing and topological strings, arXiv:0910.2615 [SPIRES].

[21] S. Cecotti, A. Neitzke and C. Vafa, R-twisting and 4d/2d correspondences, arXiv:1006.3435 [SPIRES].

[22] D. Gaiotto, G.W. Moore and A. Neitzke, Framed BPS states, arXiv:1006.0146 [SPIRES].

[23] E. Andriyash, F. Denef, D.L. Jafferis and G.W. Moore, Wall-crossing from supersymmetric galaxies, arXiv: 1008.0030 [SPIRES].

[24] J. Manschot, Stability and duality in $N=2$ supergravity, Commun. Math. Phys. 299 (2010) 651 [arXiv:0906.1767] [SPIRES].

[25] C. Itzykson and J. Zuber, Quantum field theory, McGraw-Hill, New York U.S.A. (1980).

[26] P.S. Aspinwall and D.R. Morrison, Topological field theory and rational curves, Comm. Math. Phys. 151 (1993) 245.

[27] R. Gopakumar and C. Vafa, M-theory and topological strings. I, hep-th/9809187 [SPIRES].

[28] R. Gopakumar and C. Vafa, M-theory and topological strings. II, hep-th/9812127 [SPIRES].

[29] J. Manschot, Wall-crossing of D4-branes using flow trees, arXiv:1003.1570 [SPIRES].

[30] J. Manschot, The Betti numbers of the moduli space of stable sheaves of rank 3 on P2, arXiv: 1009.1775 [SPIRES].

[31] T. Nishinaka, Multiple D4-D2-D0 on the conifold and wall-crossing with the flop, JHEP 06 (2011) 065 [arXiv: 1010.6002] [SPIRES].

[32] S. Alexandrov, D. Persson and B. Pioline, On the topology of the hypermultiplet moduli space in type-II/CY string vacua, Phys. Rev. D 83 (2011) 026001 [arXiv:1009.3026] [SPIRES].

[33] S. Alexandrov, D. Persson and B. Pioline, Fivebrane instantons, topological wave functions and hypermultiplet moduli spaces, JHEP 03 (2011) 111 [arXiv:1010.5792] [SPIRES].

[34] T. Dimofte and S. Gukov, Refined, motivic and quantum, Lett. Math. Phys. 91 (2010) 1 [arXiv:0904.1420] [SPIRES].

[35] T. Dimofte, S. Gukov and Y. Soibelman, Quantum wall crossing in $N=2$ gauge theories, Lett. Math. Phys. 95 (2011) 1 [arXiv:0912.1346] [SPIRES].

[36] F. Denef, Attractors at weak gravity, Nucl. Phys. B 547 (1999) 201 [hep-th/9812049] [SPIRES].

[37] S. Banerjee, A. Sen and Y.K. Srivastava, Partition functions of torsion $>1$ dyons in heterotic string theory on $T^{6}$, JHEP 05 (2008) 098 [arXiv:0802.1556] [SPIRES]. 
[38] A. Sen, Wall crossing formula for $N=4$ dyons: a macroscopic derivation, JHEP 07 (2008) 078 [arXiv:0803.3857] [SPIRES].

[39] A. Dabholkar, J. Gomes and S. Murthy, Counting all dyons in $N=4$ string theory, JHEP 05 (2011) 059 [arXiv:0803.2692] [SPIRES].

[40] M. Reineke, The Harder-Narasimhan system in quantum groups and cohomology of quiver moduli, Invent. Math. 152 (2003) 349.

[41] J. de Boer, S. El-Showk, I. Messamah and D. Van den Bleeken, Quantizing $N=2$ multicenter solutions, JHEP 05 (2009) 002 [arXiv: 0807.4556] [SPIRES].

[42] J. Stoppa, D0-D6 states counting and GW invariants, arXiv:0912.2923.

[43] Y. Toda, On a computation of rank two Donaldson-Thomas invariants, arXiv:0912.2507.

[44] W.-y. Chuang, D.-E. Diaconescu and G. Pan, Rank two ADHM invariants and wallcrossing, arXiv: 1002.0579 [SPIRES].

[45] C. Bachas and E. Kiritsis, $F^{4}$ terms in $N=4$ string vacua, Nucl. Phys. Proc. Suppl. 55B (1997) 194 [hep-th/9611205] [SPIRES].

[46] A. Gregori et al., $R^{2}$ corrections and non-perturbative dualities of $N=4$ string ground states, Nucl. Phys. B 510 (1998) 423 [hep-th/9708062] [SPIRES].

[47] A. Sen, Arithmetic of quantum entropy function, JHEP 08 (2009) 068 [arXiv:0903.1477] [SPIRES].

[48] A. Sen, How do black holes predict the sign of the Fourier coefficients of Siegel modular forms?, arXiv: 1008.4209 [SPIRES].

[49] A. Dabholkar, J. Gomes, S. Murthy and A. Sen, Supersymmetric index from black hole entropy, JHEP 04 (2011) 034 [arXiv:1009.3226] [SPIRES].

[50] I. Bena, C.-W. Wang and N.P. Warner, Mergers and typical black hole dicrostates, JHEP 11 (2006) 042 [hep-th/0608217] [SPIRES].

[51] J.J. Duistermaat and G.J. Heckman, On the variation in the cohomology of the symplectic form of the reduced phase space, Invent. Math. 69 (1982) 259.

[52] K. Nagao, On higher rank Donaldson-Thomas invariants, arXiv:1002.3608.

[53] E. Witten, BPS bound states of D0-D6 and D0-D8 systems in a B-field, JHEP 04 (2002) 012 [hep-th/0012054] [SPIRES].

[54] D. Maulik, N. Nekrasov, A. Okounkov, and R. Pandharipande, Gromov-Witten theory and Donaldson-Thomas theory. I, Compos. Math. 142 (2006) 1263.

[55] K. Behrend and B. Fantechi, Symmetric obstruction theories and Hilbert schemes of points on threefolds, Algebra Number Theory 2 (2008) 313.

[56] M. Levine and R. Pandharipande, Algebraic cobordism revisited, Invent. Math. 176 (2009) 63.

[57] J. Li, Zero dimensional Donaldson-Thomas invariants of threefolds, Geom. Topol. 10 (2006) 2117.

[58] E.W. Weisstein, q-exponential function, http://mathworld.wolfram.com/q-ExponentialFunction.html.

[59] J. Manschot, B. Pioline and A. Sen, A fixed point formula for the index of multi-centered $N=2$ black holes, JHEP 05 (2011) 057 [arXiv: 1103.1887] [SPIRES].

[60] B. Pioline, Four ways across the wall, in the proceedings of Algebra, Geometry and Mathematical Physics, Tjärnö, Sweden, 25-30 October 2010 [arXiv:1103.0261] [SPIRES]. 